\title{
Role of Histone Methylation in Cognition and Effects of Different Durations of Environmental Enrichment on Learning and Memory
}

\author{
Dissertation \\ for the award of the degree \\ „Doctor rerum naturalium“ \\ of Georg-August-Universität Göttingen \\ submitted by \\ Cemil Kerimoglu \\ born in Baku, Azerbaijan
}

Göttingen 2012

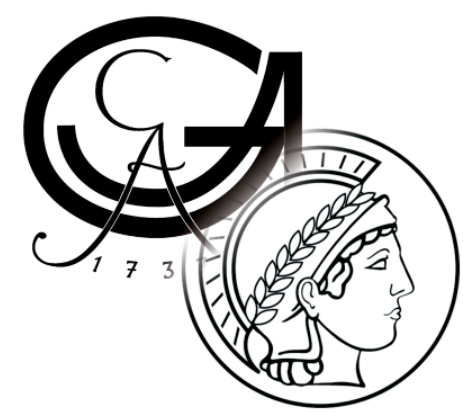




\section{PhD Thesis Committee}

1. Prof. Dr. Andre Fischer: Laboratory of Aging and Cognitive Diseases, European Neuroscience Institute, Goettingen, Germany

2. Prof. Dr. Walter Stuehmer: Department of Molecular Biology of Neuronal Signals, Max Planck Institute for Experimental Medicine, Goettingen, Germany

3. Prof. Dr. Wolfgang Fischle: Department of Chromatin Biochemistry, Max Planck Institute for Biophysical Chemistry, Goettingen, Germany

Date of Submission: 05 March 2012 


\section{Declaration}

I herewith declare that I have prepared the dissertation "Role of Histone Methylation in Cognition and Effects of Different Durations of Environmental Enrichment on Learning and Memory" entirely by myself with no other aids and sources than quoted.

Goettingen, 24.02.12

Cemil Kerimoglu 


\section{Table of Contents}

Table of Contents $i$

Summary vii

$\begin{array}{ll}\text { Abbreviations } & \text { ix }\end{array}$

$\begin{array}{ll}\text { INTRODUCTION } & 1\end{array}$

Learning and Memory 1

Memory Storage in the Brain 1

Forms of Memory 1

Declarative and Procedural Memories $\quad 2$

Short-term and Long-term Memories 2

Testing Memory in Rodents 3

Associative Memory 3

$\begin{array}{ll}\text { Spatial Memory } & 4\end{array}$

Biological and Molecular Mechanisms of Learning and Memory 4

$\begin{array}{ll}\text { Epigenetics and Chromatin Plasticity } & 7\end{array}$

$\begin{array}{ll}\text { Histone Methylation } & 8\end{array}$

Role of Epigenetic Mechanisms in Learning and Memory 11

$\begin{array}{ll}\text { Histone Methylation in Learning and Memory } & 12\end{array}$

$\begin{array}{ll}\text { Environmental Enrichment } & 12\end{array}$

$\begin{array}{lr}\text { Aims of The Project } & 14\end{array}$

$\begin{array}{ll}\text { Part } 1 & 14\end{array}$

$\begin{array}{ll}\text { Part } 2 & 15\end{array}$ 
Environmental Enrichment

$\begin{array}{ll}\text { Behavioural Analysis } & 16\end{array}$

$\begin{array}{ll}\text { Open Field Test } & 16\end{array}$

$\begin{array}{ll}\text { Elevated Plus Maze } & 17\end{array}$

$\begin{array}{ll}\text { Novel Object Recognition } & 17\end{array}$

$\begin{array}{ll}\text { Pavlovian Fear Conditioning } & 17\end{array}$

$\begin{array}{lr}\text { Morris Water Maze } & 18\end{array}$

$\begin{array}{ll}\text { Mll2 Genotyping } & 18\end{array}$

$\begin{array}{ll}\text { DNA Isolation from Tail } & 18\end{array}$

$\begin{array}{ll}\text { Polymerase Chain Reaction (PCR) } & 18\end{array}$

RNA Isolation and Quantitative Real Time PCR 19

$\begin{array}{ll}\text { RNA Isolation } & 19\end{array}$

$\begin{array}{ll}\text { Reverse Transcription } & 20\end{array}$

$\begin{array}{ll}\text { qPCR } & 20\end{array}$

$\begin{array}{ll}\text { With UPL Probes } & 20\end{array}$

With SYBR Green $\quad 22$

$\begin{array}{ll}\text { DNA Microarray } & 23\end{array}$

Chromatin Immunoprecipitation (ChIP) 24

Protein Isolation and Western Blotting $\quad 26$

$\begin{array}{ll}\text { Subcellular Fractionation } & 26\end{array}$

$\begin{array}{ll}\text { Protein Isolation with TRIZOL } & 27\end{array}$ 
$\begin{array}{ll}\text { Protein Isolation with TX Buffer } & 27\end{array}$

Determination of Protein Concentration $\quad 27$

Preparation of Protein Lysates for SDS-PAGE and Immunoblot 28

$\begin{array}{ll}\text { SDS-Page } & 28\end{array}$

$\begin{array}{ll}\text { Protein Transfer } & 28\end{array}$

$\begin{array}{ll}\text { Immunoblot } & 29\end{array}$

Immunostaining $\quad 29$

$\begin{array}{ll}\text { Confocal Microscope } & 30\end{array}$

$\begin{array}{ll}\text { Statistical Analysis } & 31\end{array}$

RESULTS

PART 1: CHARACTERIZATION PF FOREBRAIN SPECIFIC MLL2 CONDITIONAL KNOCK-OUT MICE

MIl2 expression in different brain regions

Creating forebrain specific MII2 conditional knock-out mice 32

The expression of the full-length transcript of $\mathbf{M l l 2}$ is decreased in the forebrain of F/F CKII mice

Brain morphology and body size are normal in F/F CKII mice 34

Knock-down of Mll2 in the forebrain leads to increased anxiety and learning $\begin{array}{ll}\text { deficits } & 35\end{array}$

Increased Anxiety 36

Learning Impairment 36 
Confirmation of differences in dorso-ventral gene expression pattern in hippocampus

Knock-down of MIl2 leads to deregulation of gene expression in dentate gyrus

MIl2 knock-down leads to decreased histone 3 Lysine 4 methylation (H3K4me3) at gene promoters

Injection of HDAC inhibitor SAHA does not change the expression of the genes neither in $\mathrm{F} / \mathrm{F}$ nor in $\mathrm{F} / \mathrm{F} \mathrm{CKII}$ mice

\section{PART 2: EFFECTS OF DIFFERENT DURATIONS OF ENVIRONMENTAL ENRICHMENT ON LEARNING AND MEMORY}

2 weeks of EE are enough to facilitate learning whereas 1 week of EE in not

Long-term EE has persistent effects on learning whereas short-term EE does not

Long-term EE induces a substantial change in gene expression in hippocampus

Short-term enrichment induces only a subtle change in gene expression

DISCUSSION

PART 1: CHARACTERIZATION PF FOREBRAIN SPECIFIC MLL2 CONDITIONAL KNOCK-OUT MICE 
MIl2 knock-down in the forebrain leads to increased anxiety and learning deficits

MIl2 activates transcription of genes important for neuronal function in dentate gyrus

Histone 3 Lysine 4 methylation is decreased at promoters and coding regionsof genes downregulated in dentate gyrus as a result of MIl2 knock-down

Injection of Histone Deacetylase (HDAC) inhibitor does not increase expression of genes downregulated as a result of MIl2 knock-down

Summary and Future Directions

\section{PART 2: EFFECTS OF DIFFERENT DURATIONS OF}

A certain minimum duration of environmental enrichment is required to facilitate learning

The effects of long-term enrichment are persistent whereas those of short-term enrichment fade away soon after enrichment ceases

Long-term EE leads to substantial changes in gene expression whereas suchchanges are absent after short-term EE 
$\begin{array}{lr}\text { Appendix } 1 & 113\end{array}$

$\begin{array}{lr}\text { Appendix } 2 & 120\end{array}$

$\begin{array}{lr}\text { Acknowledgements } & 125\end{array}$

$\begin{array}{ll}\text { Curriculum Vitae } & 127\end{array}$ 


\section{SUMMARY}

\section{Part 1}

Formation of stable long-term memories requires novel gene expression, and chromatin modifications have been shown to be involved in mediating the de novo gene expression which is necessary for memory formation. Although the role of histone acetylation and different histone deacetylases (HDACs) and histone acetyltransferases (HATs) in learning and memory has been studied quite extensively, the knowledge concerning the role of histone methylation - another important histone modification - in learning and memory is lacking.

I have characterized the transgenic mouse line in which the Mll2 gene is conditionally knocked down at adult stage in the forebrain excitatory neurons by the Cre-LoxP system, with Cre recombinase being expressed under CamKII promoter in order to ensure recombination specifically in forebrain glutamatergic neurons knock-out mice being denoted as "F/F CKII" hereafter). I have established that the knock-out (F/F CKII) mice have deficits in learning and memory assessed by novel object recognition, fear conditioning and water maze tests. In addition to that the F/F CKII mice were observed to have increased anxiety assessed by open field and elevated plus maze tests. Both males and females showed the same phenotype in these tests, and there was no genotype vs sex interaction. Moreover, DNA microarray revealed that the KO mice exhibit downregulation in 153 genes in dorsal dentate gyrus when compared to the controls. Interestingly such an extensive downregulation of gene expression is not observed in the dorsal CA region of the hippocampus, which partially may be explained by the observation that M112 expression is slightly higher in the dentate gyrus than in the CA in wild-type mice to begin with. Many of the genes downregulated in $\mathrm{KO}$ mice play roles in synaptic plasticity and synaptic transmission which corroborates the behavioural findings. Moreover, among genes selected for qRT-PCR confirmation some of them - Rab38, Car4, Adcy5, Gabrg3 and Tpm4 - did not show downregulation in the ventral dentate gyrus of F/F CKII mice. This decrease in gene expression in the dentate gyrus of F/F CKII mice was accompanied by a decrease Histone 3 Lysine 4 tri and dimethylation but not in monomethylation at gene promoters. And it was observed that acute SAHA injection did not lead to an increase in expression of those genes neither in control $(\mathrm{F} / \mathrm{F})$ nor in F/F CKII mice. 


\section{Part 2}

In the second part of my $\mathrm{PhD}$ work I investigated the effects of different durations of environmental enrichment (EE) on learning and memory. Although it is firmly established that environmental enrichment facilitates learning and memory, not much is known whether its effects would persist once enriched environment is removed and mice are returned to conventional housing.

I have found out that environmental enrichment operates in three stages. If its duration does not exceed a certain level EE does not improve learning at all - this was observed with 1 week of EE where mice did not show any learning improvement. After a certain level of duration EE begins to exert positive effects on learning - 2 weeks of EE were enough to facilitate learning. However, the most interesting observation was that once mice were put back into standard housing after 2 weeks of EE the learning enhancement mediated by prior enrichment soon faded away. This was however not the case if mice were enriched for a much longer time - i.e. the positive effects of 10 weeks of EE persisted for at least up to 2 months after enrichment was ceased. These different levels of persistence were accompanied by differences in gene expression that short-term ( 2 weeks) and long-term (10 weeks) EE mediated; long-term EE mediated a robust change in gene expression with 62 genes up and 69 genes downregulated, however 2 weeks of EE failed to exert such a drastic effect. 


\section{ABBREVIATIONS}

Nkapl - NFKB activating protein-like

Rab38 - RAB38, member of RAS oncogene family

Ap1s3 - adaptor-related protein complex AP-1, sigma 3

Acot 4 - acyl-CoA thioesterase 4

Dusp2 - dual specificity phosphatase 2

Adcy 5 - adenylate cyclase 5

Ptgr1 - prostaglandin reductase 1

Prkra - protein kinase, interferon inducible double stranded RNA dependent activator

Gabrg3 - gamma-aminobutyric acid (GABA) A receptor, subunit gamma 3

Car4 - carbonic anhydrase 4

Ckap4 - cytoskeleton-associated protein 4

Stxbp 2 - syntaxin binding protein 2

Syp12 - synaptophysin-like 2

Gkap1 - G kinase anchoring protein 1

Tpm4 - tropomyosin 4

Tceal1 - transcription elongation factor A (SII)-like 1

Oxr1 - oxidation resistance 1

Cuedc1 - CUE domain containing 1

Plbd1 - phospholipase B domain containing 1

Tjp2 - tight junction protein 2

Ccl6 - chemokine (C-C motif) ligand 6

Paip1 - polyadenylate binding protein-interacting protein 1

Nnt - nicotinamide nucleotide transhydrogenase

Cml5 - camello-like 5

Ccl8 - chemokine (C-C motif) ligand 8

Ing3 - inhibitor of growth family, member 3

Meox1 - mesenchyme homeobox 1 
Kat2a - K(lysine) acetyltransferase 2A

Lpcat2 - lysophosphatidylcholine acyltransferase 2

Klf1 - Kruppel-like factor 1 (erythroid)

Ccl17 - chemokine (C-C motif) ligand 17

Mamdc2 - MAM domain containing 2

Pde6h - phosphodiesterase 6H, cGMP-specific, cone, gamma 


\section{INTRODUCTION}

\section{$\underline{\text { Learning and Memory }}$}

Learning is regarded as the process of acquiring new skills and knowledge. Memory, in turn, refers to storage and the process of recalling the acquired information and skills. The questions where in the brain memories are formed and stored and what biological mechanisms are behind these processes have long intrigued scientists. With the advancement of science we are in the process of unravelling the biological and molecular mechanisms underlying learning and memory processes.

\section{Memory Storage in the Brain}

The first indication that certain parts of the brain are involved in the storage of memories came from the famous patient H.M. His medial temporal lobe was removed to treat the epilepsy that he was suffering from. After the surgery he exhibited severe anterograde amnesia characterized by the inability to form new memories (Scoville and Milner, 1957). However, the memories that he acquired before the operation were intact in a time-dependent fashion - i.e. he still had a partial loss of memories that he acquired shortly before the operation, but the more remote memories tended to be more intact. Moreover, his ability to acquire new motor skills and reasoning abilities remained intact (Corkin, 2002). This indicated for the first time that different kinds of cognitive skills and even different kinds of memories (to be explained below) are subserved by different brain regions. This was later confirmed by many studies.

\section{Forms of Memory}

Memories are divided into different subtypes depending on their mode of acquisition, processing and their persistence. 


\section{Declarative and Procedural Memories}

Memory is generally divided into two major subtypes depending on their modes of acquisition; declarative memory and procedural memory. Declarative memory is commonly referred to as the memory of facts and events (Donaldson and Tulving, 1972); e.g. the date of your birthday, the capital of Germany, the year when you started your $\mathrm{PhD}$ etc. This type of memory is acquired consciously and conscious awareness is necessary to recall it later (Donaldson and Tulving, 1972; Eichenbaum, 1997; Ullman, 2004). Due to this reason it is also called as explicit memory - you are explicitly aware of the information. Non-declarative memory, alternatively called procedural memory, is the memory of habits and skills (i.e. memory of a sequence, memory of a procedure); e.g. riding a bike or playing a violin (Fitts, 1954; Ullman, 2001; Ullman, 2004; Ullman and Pierpont, 2005). Acquisition and recall of this memory does not require any conscious awareness. For that reason it is also referred to as implicit memory. Implicit memory is the only type of memory that is manifested by invertebrates, whereas explicit memory is present only in vertebrates. In other words, implicit forms of memory are evolutionarily much older.

Hippocampus is the major brain part responsible for acquisition and storage of declarative memories (Eichenbaum, 2001), whereas formation of procedural memory depends on striatum (Kreitzer, 2009) and cerebellum (Saywell and Taylor, 2008).

\section{$\underline{\text { Short-term and Long-term Memories }}$}

Memory is divided into subtypes also according to the amount of time it remains accessible to recall. Short-term memory lasts only for a short period of time, as the name suggests - from seconds up to several minutes (Cowan, 2001). For example, remembering a phone number until you dial it and then forgetting it. Long-term memory however lasts for much longer time, from days to years up to a lifetime. Another type of memory which is being distinguished from the two mentioned above is working memory. This kind of memory is often confused with short-term memory but lately it has been considered as a distinct subtype. This type of memory remains accessible as long as a 
sequence of actions is being performed. It can be described as the memory of the entity in a sequence that comes before the entity currently being dealt with. And as one proceeds with the sequence, the entities that were left behind can be forgotten (Becker and Morris, 1999; Baddeley, 2003).

Recently acquired memories are stored in the hippocampus; however throughout time they become transferred to the cortex (Frankland et al., 2004; Maviel et al., 2004; Frankland and Bontempi, 2005; Teixeira et al., 2006; Restivo et al., 2009). Repeated reactivation of hippocampo-cortical circuitry (esp. during sleep) has been suggested to mediate this process (Pavlides and Winson, 1989; Wilson and McNaughton, 1994; Kudrimoti et al., 1999; Nadasdy et al., 1999; Maquet et al., 2000; Shimizu et al., 2000; Ribeiro et al., 2002; Huber et al., 2004; Peigneux et al., 2004; Ribeiro et al., 2004; Peigneux et al., 2006; Ji and Wilson, 2007; Euston et al., 2007; Buhry et al., 2011; Schwindel and McNaughton, 2011). Once the memory comes to the stage of being stored in the cortex it begins to be regarded as remote memory.

\section{Testing Memory in Rodents}

\section{$\underline{\text { Asscociative Memory }}$}

This type of memory is encoded through the association of two stimuli occurring in a specific sequence and at a certain interval. The most often used way to study it in rodents is afforded by Pavlovian fear conditioning paradigm (Blanchard and Blanchard, 1969). Here the rodents (either rats or mice) learn to associate a conditioned stimulus (CS), e.g. context or tone, with an unconditioned stimulus (US) which is presented in the form of a mild electrical footshock. Upon a second exposure to the CS, but without the presentation of US, the animal manifests freezing behaviour, which is an inborn behaviour that rodents show when in a potentially threatening situation. The amount of freezing corresponds to the amount of fear that the animal has at that moment, and the amount of fear that the animal manifests corresponds to the strength of the associative memory that it acquired, relating the CS to the expectation of US. Contextual fear conditioning, where CS is a context, is highly dependent on hippocampus (Kim and Fanselow, 1992). Cue- 
dependent fear conditioning, where CS is a cue in the form of a tone or an odour, is mediated through amygdala (LeDoux, 1993; LeDoux, 1994).

\section{Spatial Memory}

It is the type of memory concerning spatial orientation. Hippocampus is the main brain structure responsible for acquisition and storage of spatial memories (Rossi-Arnaud et al., 1991; Schwegler and Crusio, 1995; Crusio and Schwegler, 2005). Spatial memory in rodents can be tested by Morris water maze test (Morris, 1981). In this test, the animals are introduced into a maze filled with opaque water. Beneath the water there is a hidden platform, which for the mice is the only escape from the water. The animal learns to locate the platform with the help of visual cues provided. And training proceeds it learns the place of the platform and the time that it takes to reach it decreases. After training, the memory is tested by putting the animal into the pool but without platform this time. And the strength of the memory is indicated by the relative amount of time that it spends in the region where the platform was previously located.

\section{Biological and Molecular Mechanisms of Learning \& Memory}

As mentioned above hippocampus is the main region where new memories are acquired and retained for a certain time. Hippocampus has a regular structure along the longitudinal axis, and is divided into two major subregions - dentate gyrus and CA. CA further consists of two functionally distinct divisions called CA3 and CA1. Hippocampus receives projections from the cortical regions through perforant pathway. Entorhinal cortex sends excitatory projections to dentate gyrus through that pathway. Then granule cells in dentate gyrus send glutamatergic projections to pyramidal neurons in CA3 through mossy fiber pathway. And neurons in CA3 project to pyramidal neurons in CA1 through Schaffer-collateral pathway.

The cellular correlate of memory called long-term potentiation (LTP) was first identified in the perforant pathway of the hippocampus (Bliss and Lomo, 1973). Later the same phenomenon was identified in other glutamatergic synapses in the hippocampus - mossy 
fiber and Schaffer collateral synapses (Wheal et al., 1983; Harris et al., 1984; Higashima and Yamamoto, 1985; Reymann et al., 1985; Kamiya et al., 1988; Bradler and Barrioneuvo, 1989; Jaffe and Johnston, 1990) and also in other brain regions like cerebellum and striatum (Crepel and Jaillard, 1991; Calabresi et al., 1992; Shibuki and Okada, 1992; Quinn and Harris, 1995; Salin et al., 1996; Calabresi et al., 1997; Charpier and Deniau, 1997; Kimura et al., 1998; D’Angelo et al., 1999). The phenomenon of longterm potentiation was most extensively studied in Schaffer collateral synapses that CA3 neurons make on CA1 neurons. The basic rationale behind the phenomenon of long-term potentiation (LTP) of synaptic transmission is that stimulations of differing intensity and/or duration can produce qualitatively different outcomes. Any amount of stimulation that is below a certain threshold either will not lead to any changes in the strength of synaptic transmission or will lead to changes that are not long lasting. For example, stimulating the slice with a single $100-\mathrm{Hz}$ train will induce an early, short-term stage of LTP (early LTP), which would last only several minutes before returning to basal levels. However, applying at least four repeated $100-\mathrm{Hz}$ trains will result in a much more persistent form of LTP (late LTP) which will persist from hours to days (Martin et al., 2000). Moreover, another way through which a stimulus that would normally induce only a short lasting change can be made to induce a long-lasting change in synaptic transmission is to pair it with another stimulation. In this regard, the most common method used in slices is to apply a modulatory neurotransmitter (e.g. dopamine) or its analogs together with stimulation (Delanoy et al., 1983; Mochida and Libet, 1990; Huang and Kandel, 1995; Otmakhova and Lisman, 1996; Kusuki et al., 1997).

Principally long lasting forms of LTP differ from short lasting ones by dependence of the former on de novo gene expression and protein synthesis (Reymann et al., 1988; Frey et al., 1988; Bourtchuladze et al., 1994; Deisseroth et al., 1996; Frey and Morris, 1997; Impey et al., 1998; Tischmeyer and Grimm, 1999; Martin et al., 2000; Kandel, 2001; Bergado et al., 2003; Ramanan et al., 2005). As a result late LTP involves formation of new synapses and/or strengthening of already existing ones (Bailey and Chen, 1983; Bailey and Kandel, 1993; Malinow et al., 2000; Malinow and Malenka, 2002; Matsuzaki et al., 2004; Nagerl et al., 2004; Hayashi and Majewska, 2005; Segal, 2005). In contrast, 
early LTP involves only posttranslational modifications of already existing proteins (Martin et al., 2000; Kandel, 2001; Malenka and Bear, 2004).

Initial studies concerning synaptic plasticity were carried out in marine snail Aplysia (Kandel, 2001; Barco et al., 2006). When a light touch is applied to its siphon, the snail responds by withdrawing its siphon and gill. This natural reflex is enhanced when a stimulus is applied also to the tail. Tail stimulus induces the release of serotonin (another modulatory neurotransmitter), which in turn acts on sensory neurons and induces production of cAMP. The increase in cAMP leads to facilitation of excitatory glutamatergic connections that sensory neurons make on motor neurons (Klein et al., 1982; Castelluci et al., 1986; Dale et al., 1988).

In mouse hippocampus, LTP induction depends on the $\mathrm{Ca}^{+2}$ influx into the neuron through NMDA receptors (NMDARs) (Malenka, 1991; Malenka and Nicoll, 1993; Blitzer et al., 1995). An important phenomenon underlying LTP induction is that excitatory neurons possess two major kinds of ionotropic glutamate receptors; $\alpha$-amino3-hydroxy-5-methyl-4-isoxazole propionic acid (AMPA) receptors (AMPARs) and Nmethyl-D-aspartic acid (NMDA) receptors (NMDARs). AMPARs are permeable to $\mathrm{Na}^{+}$ and $\mathrm{K}^{+}$ions and mediate most of the depolarization that is responsible for synaptic transmission under basal conditions. NMDARs in turn are not readily permeable to ions because of the magnesium block that occludes the channel. However, upon depolarization (which is caused by the passage of cations through AMPRs) the magnesium block is removed and binding of glutamate to the NMDAR activates it leading to an influx not only of $\mathrm{Na}^{+}$but also, most crucially, of $\mathrm{Ca}^{+2}$ ions. The latter in turn associate with calmodulin. At low $\mathrm{Ca}^{+2}$ concentrations calmodulin is able to activate only CaM-dependent kinase II (Malenka, 1991; Malenka and Nicoll, 1993). And the latter in turn induces early LTP by phosphorylating AMPARs (Martin et al., 2000; Kandel, 2001; Malenka and Bear, 2004). Phosphorylation of AMPARs increases their ion conductivity, which leads to transient potentiation of synaptic transmission (Greengard et al., 1991; Keller et al., 1992; Blackstone et al., 1994; Carvalho et al., 2000). However once $\mathrm{Ca}^{+2}$ concentration passes beyond a certain threshold the resulting calcium/calmodulin complex activates CaM-dependent adenylate cyclase (Blitzer et al., 1995), which leads to cAMP production. The produced cAMP activates cAMP-dependent 
protein kinase (PKA), which in turn phosphorylates MAPK and results in activation of MAPK/ERK signalling which leads to CREB-dependent gene transcription, which in the end leads to maintenance of late LTP and is crucial for formation of long-term memory (Frey et al., 1993; English and Sweatt, 1996; English and Sweatt, 1997; Atkins et al., 1998; Sweatt, 2001; Sweatt, 2004; Thomas and Huganir, 2004; Reissner et al., 2006). Phosphorylated CREB binds to conserved cAMP response element (CRE) sequences at gene promoters and mediates the first wave of gene expression by inducing transcription of immediate early genes (IEGs) like c-Fos, Zif268/Egr-1 and Egr-2 (Impey et al., 1996; Impey et al., 1998; Taubenfeld et al., 1999; Bito et al., 1996; Deisseroth et al., 1996; Lu et al., 1999). Those IEGs in turn lead to successive waves of gene expression as a result of which the newly synthesized proteins lead to occurrence of new synapses, activation of silent ones and/or strengthening of already existing ones (see above).

\section{$\underline{\text { Epigenetics and Chromatin Plasticity }}$}

In eukaryotic cells, DNA does not exist in the cell as simply bare structure. It is rather wrapped around proteins called histones giving rise to the structure called chromatin. The condensed form of chromatin is called heterochromatin and is inaccessible to transcriptional machinery (Grewal and Moazed, 2003). Therefore the genes located in heterochromatic region usually are repressed and have a low level of transcription. However, euchromatin represents a relatively loose and open state of DNA, and hence the genes located at euchromatic regions are accessible to RNA transcription machinery and are expressed at a relatively high level.

$147 \mathrm{bp}$ of DNA is wrapped around an octamer of histone proteins forming the basic unit of chromatin called the nucleosome. This octamer unit consists of two of each four different histone proteins - H2A, H2B, H3 and H4. The histone proteins have an overall positive charge and that is what makes them attracted to negatively charged DNA. Each histone protein is composed of a central globular domain and an N-terminal tail. The latter appear to be crucial since they undergo different chemical modifications at different sites. The N-terminal tails of histones can undergo acetylation, methylation, phosphorylation, ubiquitilation and ADP-ribosylation (Strahl and Allis, 2000; Vaquero et 
al., 2003). And these post-translational modifications on histone tails regulate the attraction between DNA and histones. The modifications that reduce the positive charge of histones loosen the attractive force between histone proteins and DNA; and this in turn makes DNA more accessible to transcriptional machinery. Histone acetylation therefore always serves to activate gene expression (Kurdistani et al., 2004; Li et al., 2007). It occurs on Lysine 9 and 14 residues of H3, and on Lysine 5, 8, 12 and 16 residues of H4. Histone acetylation is catalysed by histone acetyltransferases (HATs), and acetyl marks are removed by histone deacetylases (HDACs) (Strahl and Allis, 2000). Histone methylation (to be discussed in more detail below) can be both activatory and repressive for gene expression depending on which residue it occurs (Shi and Whetstine, 2007; Shilatifard, 2008; Scharf and Imhof, 2011; Justin et al., 2010). It is catalysed by histone methyltransferases (HMTs) and histone methyl marks are removed by histone demethylases (HDMs) (Tachibana et al., 2001; Shi et al., 2004).

\section{Histone Methylation}

Like acetylation, histone methylation also occurs on lysine residues of histone tails. However differently from histone acetylation, which always involves addition of only one acetyl residue, histone methylation can occur in monomethylated, dimethylated and trimethylated forms (Schneider et al., 2005; Santos-Rosa et al., 2002; Wood et al., 2007). Each state of histone lysine methylation can either be carried out in a non-processive manner (i.e. monomethylation from non-methylated substrate, dimethylation from monomethylated substrate and trimethylation from dimethylated substrate) or it can be carried out in a processive manner with each level of histone lysine methylation occurring from non-methylated residue as the substrate (Scharf and Imhof, 2011).

Also differently from acetylation, histone methylation can serve either as an activatory or repressive mark depending on which lysine residue is methylated (Kouzarides, 2007; Shilatifard, 2006; Sims et al., 2003). For example, H3 lysine 9 methylation serves to inhibit transcription and hence is associated mostly with heterochromatic regions (Nakayama et al., 2001; Noma et al., 2001; Peters et al., 2001; Peters et al., 2002; Volpe et al., 2002). However, H3 lysine 4 methylation is exclusively associated with active 
transcription (Santos-Rosa et al., 2002; Kouzarides, 2007; Shilatifard, 2006; Sims and Reinberg, 2006). Other most prominent histone methylation sites are H3 lysine 27, which is associated with transcriptional repression (Boyer et al., 2006; Lee et al., 2006; Schwartz et al., 2006; Schüttengruber et al., 2009), and H3K36 which is associated with transcriptional activation (Bell et al., 2007; Lee and Shilatifard, 2007; Wang et al., 2007).

H3K4 methylation: As was mentioned above, H3K4 methylation always acts to facilitate transcription. H3K4 methylation is mostly localized near the transcription start sites of actively transcribed genes (Krogan et al., 2003; Ng et al., 2003) although it has been shown to be present in coding regions as well (Santos-Rosa et al., 2002). Moreover, there exist differences in the function of different levels of H3K4 methylation, with $\mathrm{H} 3 \mathrm{~K} 4$ trimethylation being responsible for induction of gene expression after stimulation and being more enriched around transcription start sites whereas $\mathrm{H} 3 \mathrm{~K} 4$ dimethylation being distributed evenly across the coding region and being responsible only for the expression of constitutively active genes (Santos-Rosa et al., 2002; $\mathrm{Ng}$ et al., 2003).

COMPASS complex was the first H3K4 methylase identified. It was identified in yeast and consists of the actual methyltransferase Set1 together with other accessory proteins (Miller et al., 2001). Set1 itself is enzymatically active only within the COMPASS complex and is able to monomethylate, dimethylate and trimethylate H3K4 (Miller et al., 2001; Roguev et al., 2001; Nagy et al., 2002). Set1 is the only H3K4 methylase in yest; however in mammals there exist six Set1-related homologs that are capable of performing H3K4 methylation - MLL1, MLL2, MLL3, MLL4, hSet1A, hSet1B (Shilatifard, 2008; Ansari and Mandal, 2010). The functions of mixed-lineage leukemia (MLL) type of $\mathrm{H} 3 \mathrm{~K} 4$ methylases consist of cell cycle regulation, development and embryogenesis (through regulating HOX gene expression) (Yu et al., 1995; Hess et al., 1997; Yagi et al., 1998; Yu et al., 1998; Terranova et al., 2006; Jude et al., 2007). Moreover, MLLs have been found to be rearranged in different types of cancers, especially leukemias - hence the name (Thomas et al., 2005; Horton and Williams, 2006; Meyer et al., 2009; Marschalek, 2010). All H3K4 methylases consist of a C-terminal catalytic SET domain which performs the actual histone methylation, PHD domains and RING fingers that are involved in protein-protein interactions and HMG domains that are 
responsible for DNA binding. Moreover, all of them exist as distinct multiprotein complexes both with common but also complex specific protein subunits. The common subunits include Ash2, Wdr5, Rbbp5 and Dpy30 (Ansari and Mandal, 2010). They are depicted in red in the picture below, whereas complex specific subunits are depicted in black.

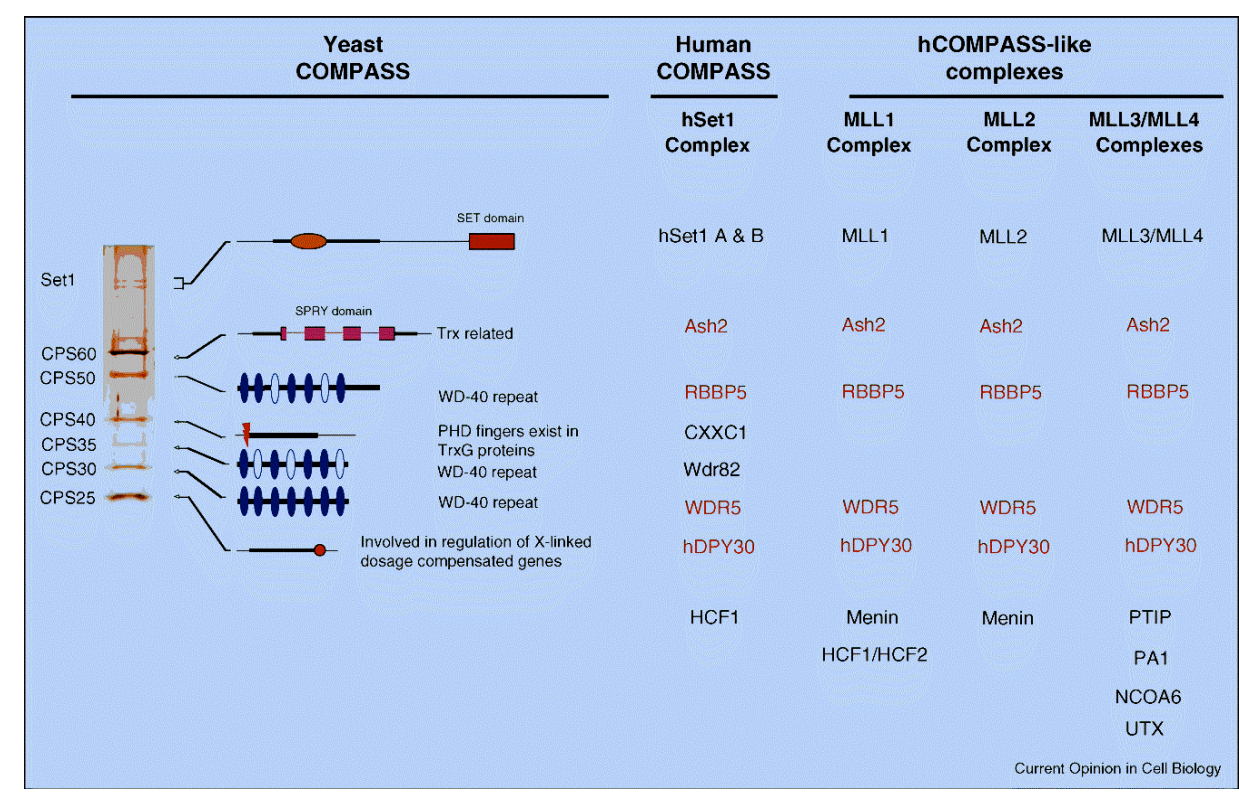

Ali Shilatifard, Current Opinion in Cell Biology, 2008

MLLs are functional histone methyltransferases only in the context of the other protein components associated with them in multiprotein complexes. And each of the components associated with MLL plays a crucial role in regulating MLL function. For example, knocking down any of the core components of MLL complexes - Wdr5, Rbbp5 or Ash2 - has been shown to decrease its H3K4 methylase activity without affecting the recruitment of MLL to gene promoters (Dou et al., 2005; Wysocka et al., 2005; Steward et al., 2006).

MLL2: Mll2 gene is a close homolog of Mll1 through gene duplication (FitzGerald and Diaz, 1999). It is highly expressed throughout development and also in adult tissue (Glaser et al., 2006; McMahon et al., 2007). Like its close homolog Mll1, Mll2 is also involved in regulating HOX gene expression (Glaser et al., 2006; Ansari et al., 2008). 
Moreover, M112 has been shown to mediate transcription at the $\beta$-globin locus in erythroid cells (Demers et al., 2007). M112 knock-down in mice before E11.5 leads to embryonic lethality, whereas knocking-down M112 after that time point does not lead to any notable pathology (Glaser et al., 2009); however loss of M1l2 leads to complete loss of spermatogenesis (Glaser et al., 2009). Furthermore, it associates with estrogen receptor alpha through menin, and regulates estrogen dependent gene expression (Mo et al., 2006; Dreijerink et al., 2006).

\section{$\underline{\text { Role of Epigenetic Mechanisms in Learning and Memory }}$}

Since histone modifications appear to be an important mechanism in regulating gene expression, and since gene expression is crucial for formation and retention of long-term memories neuroscientists have long wondered whether epigenetic mechanisms play a role in memory formation. And indeed, up to date many studies have conclusively shown that histone modifications in different brain regions (especially hippocampus) are important for learning and memory. Most of the studies have used Pavlovian fear conditioning paradigm to investigate this and have shown that it induces an increase in histone acetylation (H3K9, H3K14, H4K5, H4K8 and H4K12) (Levenson et al., 2004: Chwang et al., 2007; Lubin et al., 2008; Peleg et al., 2010), histone methylation (H3K4) (Gupta et al., 2010) and histone phosphorylation (H3S10) (Chwang et al., 2006) in hippocampus. Moreover, apart from acquisition and consolidation, reconsolidation of memory after retrieval and extinction of fear memory were also shown to depend on histone acetylation and phosphorylation (Bredy et al., 2007; Lubin and Sweatt, 2007). Supporting these findings there were also many studies that showed that interfering with the mechanisms mediating chromatin modifications affects long-term potentiation (LTP), which is considered as a biological correlate of memory (see above). Increasing histone acetylation through the usage of HDAC inhibitors has been shown to facilitate memory formation and enhance LTP (Levenson et al., 2004). Moreover mice having their CREB binding protein (CBP) - a HAT - knocked out manifest deficits in learning \& memory and LTP (Alarcon et al., 2004; Valor et al., 2011). In line with these findings, mice that 
have their HDAC2 deleted manifest memory enhancement and an increase in LTP (Guan et al., 2009). Moreover inhibition of PP1, which dephosphorylates histones, has also been shown to facilitate memory (Koshibu et al., 2009).

\section{Histone Methylation in Learning and Memory}

Although the role of histone acetylation in memory formation has been extensively studied, the involvement of histone methylation, another important histone modification, in learning and memory has been relatively ignored. Only recently has its importance for learning \& memory and behaviour in general begun to be revealed. For example knock down of H3K9 methyltransferase GLP/G9a in adult neurons in mice leads learning impairment accompanied with decrease in exploratory behaviour and motivation and a decrease in anxiety (Schaefer et al., 2009). Moreover, it has recently been shown that mice having a deletion in one of the alleles of Mll1 manifest impairment in fear conditioning and increased anxiety (Gupta et al., 2010).

\section{Environmental Enrichment}

Environmental enrichment refers to provision of conditions that enhance cognitive, motor and sensory stimulation compared to standard (i.e. control) conditions. Normally, rodents are housed in standard home cages which include ad libitum access to food and water, bedding and nesting material. Environmental enrichment is achieved through placing the animals (rats or mice) in more spacious cages containing toys of different size, shape, colour and texture. Moreover, in most studies enriched environment also includes provision of running wheels in order to provide the rodents with an opportunity for

voluntary exercise as well, together with cognitive and sensory stimulation provided by the toys. In some cases, social interaction is also utilized as an additional component of the enriched environment, with control animals housed singly in standard home cages and enriched animals housed in groups. However, in many studies, including the one to be presented in this thesis, the effect of social interaction as an additional factor is 
eliminated by housing control mice in groups as well. This provides the researcher with an opportunity to discern the effects of only cognitive and motor stimulation on behavioural changes in rodents and on the changes in molecular pathways in their brains. Environmental enrichment as an experimental paradigm was first described by Donald Hebb (Hebb, 1947). He observed that rats that he took home as pets and allowed to roam freely showed improvements in different behavioural tasks when compared to the ones kept in the laboratory. The enriched environment he provided included two most important features which would constitute all the environmental enrichment protocols in subsequent studies, namely novelty and complexity (van Praag et al., 2000; Nithianantharajah and Hannan, 2006). Complexity is achieved by providing different toys of different size, shape, texture and colour (although colour complexity is the least effective due to limitations in rodent vision). Novelty is achieved by changing the toys at a regular basis; this includes removing some of the old toys and replacing them with new ones and rearranging the remaining toys inside the cage. Increased complexity and novelty leads to increased levels of stimulation in different brain regions - e.g. changing the objects and their position provides additional cognitive stimulation by facilitating the formation of new spatial maps in hippocampus (Nithianantharajah and Hannan, 2006).

The first studies investigating the effects of enriched environment on rodent brain documented that environmental enrichment increased brain weight and cortical thickness (Bennett et al., 1969; Diamond et al., 1972; Diamond et al., 1976). Later studies showed that enrichment increases dendritic density and the number of synaptic arborizations (Greenough and Volkmar, 1973; Greenough et al., 1985; Turner et al., 1985; Rampon et al., 2000; Faherty et al., 2003; Leggio et al., 2005). In line with induction of increased synaptogenesis, environmental enrichment also increases the expression of post and presynaptic proteins, PSD95 and synaptophysin respectively (Frick et al., 2003; Tang et al., 2001). Moreover, environmental enrichment has also been shown to increase the expression of NMDA and AMPA receptors (Tang et al., 2001; Naka et al., 2005) together with enhancing LTP (Foster et al., 2001; Duffy et al., 2001; Artola et al., 2006). Last but not least, it has been shown that environmental enrichment facilitates adult neurogenesis in hippocampus, through both increasing the number of newborn neurons 
and also facilitating their survival (Kempermann et al., 1997; Kempermann et al., 1998a; Kempermann et al., 1998b; Bruel-Jungermann et al., 2005).

Through all these, environmental enrichment leads to alterations in behaviour. It facilitates learning and memory in young adult mice (Rampon et al., 2000; Tang et al., 2001; Duffy et al., 2001; Lee et al., 2003), ameliorates memory deficits in aged and Alzheimer's disease model mice (Bennett et al., 2006; Fischer et al., 2007) and decreases anxiety (Roy et al., 2001; Benaroya-Milshtein et al., 2004). It is mostly believed that the effects of environmental enrichment on brain structure and function, and on behaviour are mediated through cellular and molecular mechanisms underlying learning and synaptic plasticity (Rosenzweig and Bennett, 1996; van Praag et al., 2000; Nithianantharajah and Hannan, 2006). The effect of increased adult hippocampal neurogenesis on learning enhancement remains more controversial since some studies have claimed that adult neurogenesis is not crucial for learning \& memory (Meshi et al., 2006; Jaholkowsky et al., 2009).

\section{AIMS OF THE PROJECT}

\section{Part 1}

My $\mathrm{PhD}$ consisted of two main parts. In the first part I characterized behaviourally and molecularly transgenic mice having their Mll2 gene knocked out in forebrain excitatory neurons. M112 is a histone methyltransferase responsible for $\mathrm{H} 3 \mathrm{~K} 4$ methylation and a close homolog of M111 (see above), the heterozygous knock-out of which has previously been shown to produce deficits in learning and in LTP (Kim et al., 2007; Gupta et al., 2010). However, those studies did not perform a comprehensive analysis of the role that Ml11 might play in regulating the molecular mechanisms involved in neuronal function and synaptic plasticity. Moreover, the heterozygous knock-out mice used in those studies were straight knock-outs. Therefore, the behavioural and synaptic deficits observed in transgenic mice cannot be attributed solely to the role of Mll1 in the functioning in adult forebrain excitatory neurons which are the ones mainly involved in sophisticated cognitive functions like learning \& memory. Therefore by choosing to study the effect of 
M112 knock-down in excitatory forebrain neurons in adults I was aiming to achieve two major goals: (i) to extend our knowledge on the role of histone methylation in learning \& memory by studying the involvement of another histone methyltransferase in those processes, and (ii) to implement a more specific approach by characterizing the transgenic mice that have their Mll2 gene knocked-out only in a subset of neurons at adult stage.

\section{Part 2}

In the second part of my $\mathrm{PhD}$ I set out to investigate the effects of different durations of environmental enrichment on learning \& memory with an attempt to uncover the molecular mechanisms behind those effects. Although it has long been known that environmental enrichment facilitates learning, a comprehensive analysis of the effects of different durations of environmental enrichment on learning \& memory has never been performed. Moreover, knowledge concerning persistence of the effects of environmental enrichment once enrichment ceases is currently scarce; therefore one of my main aims was to find out what amount of enrichment is enough not only to facilitate learning but also exert persistent effects once it ceases. Last but not least, I also tried to find out whether different durations of environmental enrichment activate different molecular pathways in hippocampus. In my investigations, I concentrated on two different durations of enrichment: (i) 2 weeks (short-term) and (ii) 10 weeks (long-term). 


\section{MATERIALS AND METHODS}

\section{Animals}

Male mice were single-housed and female mice were group-housed in standard home cages (365 x 207 x $140 \mathrm{~mm}$ ). Food and water were provided ad libitum. All experiments were performed according to the animal protection law and were approved by the District Government of Germany. All mice were of C57B/6J background. The generation of Mll2 F/F and CamKII-Cre mice was described previously (Glaser et al., 2004; Minichiello et al., 1999).

In brief, one week before the start of the behavioural battery the animals were allocated to cages and put into the room where the behavioural experiments were to be performed. Male mice were housed singly while the female mice were housed in groups of 4-5 in order to have their oestrous cycle synchronized. The behavioural battery was always performed in the following sequence: Open Field Test, Novel Object Recognition Test, Elevated Plus Maze, Contextual Fear Conditioning, Morris Water Maze.

\section{Environmental Enrichment}

The mice subjected to enriched environment were housed in more spacious cages $(595 \mathrm{x}$ $380 \times 200 \mathrm{~mm}$ ), 3-5 mice per cage. Apart from that the mice were provided with different objects (e.g. tunnels, castles, running wheels etc). Every day 2 of the toys were exchanged for the new ones and the ones remaining in the cage were rearranged. Food and water were provided ad libitum.

\section{Behavioural Analysis}

Open Field Test: In the open field test mice were placed near one of the side walls of a plastic arena (length $1 \mathrm{~m}$; width $1 \mathrm{~m}$; transparent side walls $20 \mathrm{~cm}$ high) and were left to explore it for $5 \mathrm{~min}$. Behaviour was recorded by a camera and analysis was performed 
using the VideoMot2 software (TSE Systems). The open field arena was cleaned with $70 \%$ ethanol before each mouse was put into the setup. The open field arena was virtually divided into 16 quadrants, and the relative time spent in the central 4 quadrants was plotted as a read-out for anxiety. Moreover, the total distance travelled in the arena was also analyzed.

Elevated Plus Maze: The Plus Maze setup was made of plastic and was $53 \mathrm{~cm}$ above the ground level. It consisted of two open and two closed arms $45 \mathrm{~cm}$ long and $10 \mathrm{~cm}$ wide, which came off from the center region $10 \times 10 \mathrm{~cm}$. The closed arms were bordered with sidewalls $29 \mathrm{~cm}$ high. Mice were put into the center facing one of the open arms. The behavioural performance was recorded during a $5 \mathrm{~min}$ period by VideoMot2 (TSE Systems).

Novel Object Recognition: For the novel object recognition test mice were habituated to an empty plastic arena for 5 min for 2 consecutive days, the exploratory pattern obtained on the first day of habituation being used as a read-out for the open field exploration. For the next two days mice were presented with two white boxes $(4.5 \times 6.5 \times 4 \mathrm{~cm})$, which were spaced $52 \mathrm{~cm}$ away from each other, and $18 \mathrm{~cm}$ away from the sidewalls. The mice were left to explore them for $5 \mathrm{~min}$. On the training day the mice were introduced to two black cubes and left to explore them for $5 \mathrm{~min}$. After that they were put back into the home cage and retained there for $5 \mathrm{~min}$. After the $5 \mathrm{~min}$ retention they were reintroduced into the plastic arena with one of the black cubes being exchanged to a small stone. They were allowed to explore the objects for $5 \mathrm{~min} .24 \mathrm{hr}$ later the mice were reintroduced into the plastic arena and presented with the same black cube and a red tape. They were allowed to explore the objects for $5 \mathrm{~min}$. Before the mice were put into the arena, the latter was cleaned with $70 \%$ ethanol.

Pavlovian Fear Conditioning: Fear conditioning test was performed using TSE fear conditioning system. The setup consisted of a computer-based control unit, with a shock generator, connected to the training chamber. The latter consisted of metal grids at the bottom, a 10 Watt light at the ceiling, a fan and a loudspeaker. The walls of the chamber 
consisted of a Plexiglas box with measurements of $25 \times 25 \times 25 \mathrm{~cm}$. Mice were allowed to explore the chamber for $3 \mathrm{~min}$ after which they received a mild electric foot shock (constant current, $0.5 \mathrm{~mA}$ ) for $2 \mathrm{sec}$. The next day (i.e. $24 \mathrm{hr}$ later) they were introduced to the same context for 3 min without receiving a footshock.

Morris Water Maze: The training was performed in a circular pool (diameter $1.2 \mathrm{~m}$ ) filled with opaque water. A platform $(11 \mathrm{x} 11 \mathrm{~cm})$ was submerged into the water in the center of one of the quadrants (target quadrant). Moreover there were different visual cues on four sites of the pool. Water level was $35 \mathrm{~cm}$ and the temperature was between $18-20^{\circ} \mathrm{C}$. The swimming behaviour of the mice was recorded by a camera and was analyzed by VideoMot2 (TSE). At each training session the mice were placed into the maze subsequently from four random points and were allowed to swim (i.e. search for the platform) for $60 \mathrm{sec}$. If during the $60 \mathrm{sec}$ the mouse failed to find the platform it was gently guided to it. Mice were allowed to stay on the platform for $15 \mathrm{sec}$. Mice were subjected to the probe test $24 \mathrm{hr}$ after the last training session. In the probe test the platform was removed and the mice were placed into the maze at one of those four points furthest away from the platform. They were allowed to swim for $60 \mathrm{sec}$.

\section{Mll2 Genotyping}

DNA Isolation from Tail: A small part of mouse tail tissue was kept in $1.5 \mathrm{ml}$ tubes (Eppendorf, Hamburg, Germany). In order to isolate the tail DNA $200 \mu$ l of DirectPCR ${ }^{\circledR}$ Lysis Reagent (Peqlab, Erlangen, Germany) were added into the tube together with 0.3 $\mathrm{mg} / \mathrm{ml}$ Proteinase K (Peqlab, Erlangen, Germany). The tubes were then incubated on a thermomixer (Eppendorf, Hamburg, Germany) at $55^{\circ} \mathrm{C}$ and shaking for $16 \mathrm{~h}$ at $550 \mathrm{rpm}$. After that the temperature was raised to $85^{\circ} \mathrm{C}$ and the tubes were incubated at the latter temperature for $45 \mathrm{~min}$. The DNA samples were then briefly spun down and were kept at $4^{\circ} \mathrm{C}$ throughout. $1 \mu \mathrm{l}$ of tail DNA was used for subsequent polymerase chain reaction.

Polymerase Chain Reaction (PCR): The PCR to detect the Mll2 knock-out was performed using the primers encompassing the region containing the exon 2 with the Flox sites at its 
borders, the Dream Taq Polymerase (Fermentas, Heidelberg, Germany), Dream Tag Buffer (Fermentas, Heidelberg, Germany) and the dNTP mix (Fermentas, Heidelberg, Germany). In order to detect the Cre transgene the corresponding primers were also added to the reaction mix. The reaction mix together with the tail DNA were pipetted into in $0.2 \mathrm{ml} \mathrm{micro} \mathrm{test} \mathrm{tubes} \mathrm{(Nerbe} \mathrm{Plus,} \mathrm{Winsen/Luhe,} \mathrm{Germany),} \mathrm{and} \mathrm{the} \mathrm{reaction} \mathrm{was}$ performed in a Mastercycler ep gradient S (Eppendorf, Hamburg, Germany). The PCR reaction mix protocol and the program are explained below.

Dream Taq Buffer (Green): $2.5 \boldsymbol{\mu l}$

dNTP Mix $(2.5 \mathrm{mM}): \mathbf{2} \boldsymbol{\mu l}$

loxP Primer Forward: $\mathbf{0 . 2 5} \boldsymbol{\mu l}$

loxP Primer Reverse: $\mathbf{0 . 2 5} \boldsymbol{\mu l}$

CamK-Cre Primer Forward: $\mathbf{0 . 1 3} \boldsymbol{\mu l}$

Cam-Cre Primer Reverse: $\mathbf{0 . 1 3} \boldsymbol{\mu l}$

PCR-grade Water: $\mathbf{1 8 . 5 4} \boldsymbol{\mu l}$

Dream Taq: $\mathbf{0 . 2} \boldsymbol{\mu l}$

Tail DNA: $1 \boldsymbol{\mu l}$

$95^{\circ} \mathrm{C}-5 \mathrm{~min}$

$95^{\circ} \mathrm{C}-30 \mathrm{sec}$

$58^{\circ} \mathrm{C}-30 \mathrm{sec}$

$68^{\circ} \mathrm{C}-1 \mathrm{~min}$

$68^{\circ} \mathrm{C}-7 \mathrm{~min}$

Here are the sequences of the primers used for genotyping:

\begin{tabular}{|l|l|c|}
\hline \multicolumn{1}{|c|}{ Primer } & \multicolumn{1}{c|}{ Sequence (5'-3') } & $\mathbf{T m}^{\mathbf{0}}$ \\
\hline loxPse Forward & gtcctgtgttcagtccaaggtag & 57 \\
\hline loxPas Reverse & ggagaacagttgtggggagatgggtc & 63 \\
\hline CamK-Cre Forward & gggaggtaggaagagcgatg & 56 \\
\hline CamK-Cre Reverse & ccatgagtgaacgaacctgg & 54 \\
\hline
\end{tabular}

\section{RNA Isolation and Quantitative real time PCR (qPCR)}

RNA Isolation: The dissection of dentate gyrus and CA regions was performed under a stereomicroscope (Motic) as described previously (Hagihara et al., 2009). The RNA from dorsal \& ventral dentate gyrus and dorsal \& ventral CA were isolated using TRIZOL Reagent from Invitrogen. For RNA isolation from whole hippocampus, prefrontal cortex 
and cerebellum the protocol was adjusted with the amounts written in parantheses in red (see also for protein isolation). The tissue was homogenized in 400 (1000) $\mu 1$ of TRIZOL Reagent. After that 80 (400) $\mu 1$ of chloroform was added to the samples and the solutions were left at room temperature (RT) for $15 \mathrm{~min}$. Later the samples were centrifuged at $12000 \mathrm{~g}$, at $4^{\circ} \mathrm{C}$ for $15 \mathrm{~min}$. The resulting aqueous phase was collected, mixed with 200 (500) $\mu \mathrm{l}$ of Isopropanol and kept at $-20^{\circ} \mathrm{C}$ for at least $30 \mathrm{~min}$. The rest (i.e. the interphase and organic phenol-chloroform phase) was frozen at $-80^{\circ} \mathrm{C}$ for subsequent protein isolation (see below). Afterwards the aqueous phase + isopropanol mixture was centrifuged for $30 \mathrm{~min}$ at $4^{\circ} \mathrm{C}$ at $12000 \mathrm{~g}$. The pellet was dissolved in 30-50 (100) $\mu \mathrm{l}$ RNase free water.

Reverse Transcription: cDNA was synthesized using the Transcriptor First Strand cDNA Synthesis Kit (Roche Applied Science, Mannheim, Germany). $1 \mu \mathrm{g}$ of RNA was mixed with $2 \mu \mathrm{l}$ of random hexamers $(600 \mathrm{pmol} / \mu \mathrm{l})$ and with $\mathrm{dH}_{2} \mathrm{O}$ (PCR-grade) up to a volume of $13 \mu \mathrm{l}$ in a $200 \mu \mathrm{l}$ tube (Nerbe Plus, Winsen/Luhe, Germany). The mixture was incubated at $65^{\circ} \mathrm{C}$ for $10 \mathrm{~min}$ in a Mastercycler ep gradient $\mathrm{S}$ (Eppendorf, Hamburg, Germany) to denature the RNA into primary structure. After that $0.5 \mu 1$ of Reverse Transcriptase $(20 \mathrm{U} / \mu \mathrm{l}), 2 \mu \mathrm{l}$ of Deoxynucleotide Mix (10 mM each), $4 \mu 1$ of the $5 \mathrm{X}$ Transcriptor Reverse Transcriptase Reaction Buffer and $0.5 \mu 1$ Protector RNase Inhibitor $(40 \mathrm{U} / \mu \mathrm{l})$ were added to the tube. The final mixture was incubated at $25^{\circ} \mathrm{C}$ for $10 \mathrm{~min}$ followed by incubation at $55^{\circ} \mathrm{C}$ in the Mastercycler. After that the Reverse Trascriptase was inactivated by incubating at $85^{\circ} \mathrm{C}$ for $5 \mathrm{~min}$ in the same Mastercycler. The cDNA was stored at $4^{\circ} \mathrm{C}$.

qPCR: a) With UPL Probes: qPCR was performed in a Roche 480 Light Cycler (Roche Applied Science, Mannheim, Germany) using the primers from the Roche Universal Probe Library (UPL) together with specific fluorescence probes and a Light Cycler PCR Mix (Roche Applied Science, Mannheim, Germany). The primers and the corresponding UPL probes are listed in the table below. $10 \mu \mathrm{l}$ from each cDNA sample was diluted 10fold with PCR-grade water. And the rest $(10 \mu \mathrm{l})$ was pooled into one tube and then diluted serially to yield the following standard dilutions: $1: 1 ; 1: 2 ; 1: 4 ; 1: 8 ; 1: 16$. The 
standard dilutions were used to generate a standard curve of fluorescence intensity versus cDNA concentration. The 1:16 dilution was also used as a positive calibrator. The following were the protocol for PCR mix and the PCR program:

PCR-grade Water: $\mathbf{5 . 0 5} \boldsymbol{\mu l}$

Forward Primer $(20 \mu \mathrm{M}): \mathbf{0 . 1 5} \boldsymbol{\mu l}$

Reverse Primer $(20 \mu \mathrm{M}): \mathbf{0 . 1 5} \boldsymbol{\mu l}$

LC480 Probe Master Mix (2X): $7.5 \mu l$

UPL Probe: $\mathbf{0 . 1 5} \boldsymbol{\mu l}$

Template cDNA: $\mathbf{2} \boldsymbol{\mu l}$

Total Volume: $\mathbf{1 5} \boldsymbol{\mu l}$

$95^{\circ} \mathrm{C}-5 \mathrm{~min}$

$95^{\circ} \mathrm{C}-10 \mathrm{sec}$

$60^{\circ} \mathrm{C}-30 \mathrm{sec}$

$72^{\circ} \mathrm{C}-10 \mathrm{sec}$

$40^{\circ} \mathrm{C}-30 \mathrm{sec}$

The cDNA template and the master mix were pipetted into the wells in a 96-well plate. The plate was then sealed with the cellophane foil and spun at $1250 \mathrm{rpm}$ for $1 \mathrm{~min}$ in a centrifuge (Eppendorf, Hamburg, Germany) before being put into the Light Cycler in order to start the PCR reaction. The housekeeping gene hypoxantine guanine phosphoribosyltransferase 1 (Hprtl) was taken as an internal control.

qPCR with this protocol was performed in order to validate the results from DNA microarray and to detect Mll2 and Mll1 expression.

\begin{tabular}{|l|l|c|c|}
\hline \multicolumn{1}{|c|}{ Primer } & \multicolumn{1}{c|}{ Sequence (5'-3') } & Tm $^{\mathbf{0}}$ & UPL Probe \\
\hline Nkapl left & cacacctctcaagatgagaaacc & 63.9 & 38 \\
\hline Nkapl right & agccattgctgcaccttc & 64.1 & 38 \\
\hline Rab38 left & ccaaaacttctcctcgcact & 63.3 & 49 \\
\hline Rab38 right & tcatgtttccaaatctttcttgac & 62.9 & 49 \\
\hline Ap1s3 left & ggaccagcagcttcattgac & 64.8 & 17 \\
\hline Ap1s3 right & tgcacagcaaaaatataaactagca & 63.3 & 17 \\
\hline Acot4 left & atgcttcgacatccaaaggt & 63.4 & 17 \\
\hline Acot4 right & ggaagccatgatcagacagac & 63.7 & 17 \\
\hline Dusp2 left & gaagataaccagatggtggagataa & 63.0 & 79 \\
\hline Dusp2 right & cccactattcttcaccgagtctat & 63.3 & 79 \\
\hline Adcy5 left & cgggagaaccagcaacag & 64.6 & 18 \\
\hline Adcy5 right & ctccatggcaacatgacg & 64.1 & 18 \\
\hline Ptgr1 left & gactgagctccacccttaaa & 64.3 & 18 \\
\hline
\end{tabular}




\begin{tabular}{|l|l|l|l|}
\hline Ptgr1 right & gtaaggatccacagagaggaaca & 63.3 & 18 \\
\hline Prkra left & gcgagcaaggctttaacataa & 63.0 & 17 \\
\hline Prkra right & agacactgatactgtccgttgg & 63.0 & 17 \\
\hline Gabrg3 left & ggctcactggatcaccaca & 65.1 & 17 \\
\hline Gabrg3 right & ggcactctgcattgatagtgag & 63.7 & 17 \\
\hline Car4 left & aaaccaaggatcctagaagcagt & 63.0 & 1 \\
\hline Car4 right & gacaatgttgatgggggact & 63.6 & 1 \\
\hline Ckap4 left & ggaggaggtccagcaggt & 64.5 & 7 \\
\hline Ckap4 right & ttgcagggattggacctt & 63.1 & 7 \\
\hline Stxbp2 left & tcttgtcatcctgttgtaaaatgtc & 62.9 & 9 \\
\hline Stxbp2 right & ccgtttgttgatgtcttcca & 63.6 & 9 \\
\hline Syp12 left & tctatggggctggctaacct & 64.6 & 99 \\
\hline Syp12 right & cagcccacaggaagaagttg & 64.8 & 99 \\
\hline Gkap1 left & cagaaggagtcacgggaaga & 64.4 & 94 \\
\hline Gkap1 right & ttcaaacatttcagaggtcagc & 63.1 & 94 \\
\hline Tpm4 left & cgaccgcaagtatgaggag & 63.4 & 108 \\
\hline Tpm4 right & tcagatacctccgcctct & 63.8 & 108 \\
\hline E2f1 left & tgccaagaagtccaagaatca & 64.6 & 5 \\
\hline E2f1 right & cttcaagccgcttaccaatc & 63.5 & 5 \\
\hline
\end{tabular}

b) With SYBR Green: The qPCR was performed in a similar fashion to the one described above with the exception that in this case the reaction mix contained the SYBR Green dye (Roche Applied Science, Mannheim, Germany) was used to detect amplification instead of UPL probes. This protocol was used in order to confirm the loss of exon 2 in F/F CKII mice through recombination and for comparison of histone methylation in the promoter region (for some in the coding region - see 'Results') of the genes downregulated in F/F CKII mice, between the latter and F/F mice (see section "Chromatin Immunoprecipitation (ChIP)"). The following protocol and PCR program were used:

PCR-grade Water: $\mathbf{3} \boldsymbol{\mu l}$ Forward Primer: $0.75 \mu l$ Reverse Primer: $0.75 \mu l$ LC480 SYBR Green I Mastermix (2X): $7.5 \mu \mathbf{l}$ Template cDNA: $\mathbf{3} \boldsymbol{\mu l}$ 
$95^{\circ} \mathrm{C}-5 \mathrm{~min}$

$95^{\circ} \mathrm{C}-10 \mathrm{sec}$

$58^{\circ} \mathrm{C}-15 \mathrm{sec}$

$72^{\circ} \mathrm{C}-10 \mathrm{sec}$

$95^{\circ} \mathrm{C}-15 \mathrm{sec}$

$67^{\circ} \mathrm{C}-30 \mathrm{sec}$

$95^{\circ} \mathrm{C}-$ Acquisition

$40^{\circ} \mathrm{C}-10 \mathrm{sec}$

The following primers were used for detection of recombination of exon 2 of Mll2. The rest will be mentioned in the section "Chromatin Immunoprecipitation (ChIP)".

\begin{tabular}{|l|l|c|}
\hline \multicolumn{1}{|c|}{ Primer } & \multicolumn{1}{|c|}{ Sequence (5'-3') } & $\mathbf{T m}^{\mathbf{0}}$ \\
\hline Primer Exon 1 & cggaggaagagagcagtgagc & 58 \\
\hline Primer Exon 2 & gggaccgaagcgcagagc & 57 \\
\hline
\end{tabular}

\section{DNA Microarray}

DNA microarray was carried out as mono-color experiment. Total RNA was labeled with Cy3 according to Agilent's Low RNA Input Fluorescent Linear Amplification Kit and later hybridized to Agilent Whole Mouse Genome 4x44K G4122F microarrays according to the manufacturer's protocol. Quantity and Cy-dye incorporation rates of the generated target material were assessed using a NanoDrop ND-100. Washes were performed according to the Agilent Technologies SSPE protocol (v2.1) - wash solution 3 was replaced by acetonitrile. After that scanning was performed using an Agilent G2505B scanner. Intensity data were extracted using Agilent's Feature Extraction (FE) software, version 9.5.3.1, and analyzed using the Limma (Smyth, 2004) package of Bioconductor (Gentelman, 2004). The microarray data analysis consisted of three steps; (i) betweenarray normalization followed by (ii) fitting the data to a linear model and finally (iii) detection of differential gene expression. In order to assure that the intensities had similar distributions across arrays, VSN normalization (Huber et al., 2002) was applied to the intensity values as a method for between-array normalization. To estimate the average group values for each gene and assess differential gene expression, a simple linear model was fit to the data, and group-value averages and standard deviations for each gene were obtained. To find genes with significant expression changes between groups, empirical 
Bayes statistics were applied to the data by moderating the standard errors of the estimated values (Smyth, 2004). P-values were obtained from the moderated t-statistic and corrected for multiple testing with Benjamini-Hochberg method (Benjamini and Hochberg, 1995). For each gene, the null hypothesis, that there is no differential expression between degradation levels, was rejected when its adjusted p-value was lower than 0.05 .

\section{Chromatin Immunoprecipitation (ChIP)}

Chromatin immunoprecipitation was performed using the Low Cell ChIP Kit from Diagenode (Liege, Belgium). $11 \mu \mathrm{l}$ of magnetic beads were mixed together with $22 \mu 1$ of Buffer A into each tube. The tubes were then placed on a magnetic rack and were left there for $1 \mathrm{~min}$. After that the supernatant was removed and $22 \mu \mathrm{l}$ of Buffer A were added. The tubes were again left on a magnetic rack for 1 min and the supernatant was removed. The beads were then resuspended in $11 \mu 1$ Buffer A. After that $90 \mu 1$ of Buffer A was aliquoted into $0.2 \mathrm{ml}$ tubes (Nerbe Plus, Winsen/Luhe, Germany), and $10 \mu \mathrm{l}$ of bead + Buffer A solution and $4 \mu$ of antibody were added into each tube. The tubes were then placed in a $15 \mathrm{ml}$ falcon tube (Greiner Bio-One, Solingen, Germany) and incubated on a rotating wheel (Barloworld Scientific, Staffordshire, UK) at $4^{\circ} \mathrm{C}$ for $2 \mathrm{~h}$ at $20 \mathrm{rpm}$. In the meantime the tissue was homogenized $500 \mu$ distilled Dulbecco's PBS (DPBS henceforth; Pan Biotech, Aidenbach, Germany) + Protease Inhibitor (Roche Applied Science, Mannheim, Germany) on ice. After that $13.5 \mu 1$ of $37 \%$ Formaldehyde were added to the mixture and the tubes were incubated on a thermomixer (Eppendorf, Hamburg, Germany) at $22^{\circ} \mathrm{C}$ for $15 \mathrm{~min}$ at $500 \mathrm{rpm}$. Later $57 \mu 1$ of $1.25 \mathrm{M}$ Glycine were added and the tubes were incubated for an additional $5 \mathrm{~min}$ on the thermomixer at abovementioned conditions. After that the tubes were centrifuged for $10 \mathrm{~min}$ at $3000 \mathrm{rpm}$ at $4^{\circ} \mathrm{C}$, the supernatant was removed and the pellet was washed twice with $500 \mu \mathrm{LPBS}+\mathrm{PI}$ - i.e. centrifuged for $10 \mathrm{~min}$ at $3000 \mathrm{rpm}$. Then the pellet was resuspended in $130 \mu \mathrm{l}$ Buffer B + PI solution (200V Buffer B : 1V PI) and left on ice for 5 min. After that the samples were subjected to sonication in Bioruptor (Diagenode, Liege, Belgium) - $30 \mathrm{sec}$ $\mathrm{ON} / \mathrm{OFF}$, HIGH, 2x10 min $+3 \times 5$ min. After shearing, $870 \mu \mathrm{l}$ of Buffer A + PI (200V 
Buffer A : 1V PI) solution was added to the sheared chromatin. After that $100 \mu$ of the chromatin samples were added to the bead + Buffer A + antibody solutions $(200 \mu 1)$ that had been incubated for $2 \mathrm{~h}$ at $4^{\circ} \mathrm{C}$ on a rotating wheel. The samples were then incubated again on the rotating wheel under the same conditions overnight. At the same time additional $100 \mu \mathrm{l}$ of each chromatin sample were kept as input at $4^{\circ} \mathrm{C}$. The next day the beads were washed thrice with Buffer A - placed on magnetic rack for 1 min, supernatant removed and the pellet (beads) resuspended in $100 \mu 1$ of Buffer A. After these washes the beads were also washed once with Buffer $\mathrm{C}$ in the same way. Then the beads were resuspended in DNA Isolation Buffer (DIB) + Proteinase K $(100 \mu \mathrm{l}$ DIB $+1 \mu 1$ Proteinase K). The beads were transferred into $1.5 \mathrm{ml}$ tubes and $1 \mu 1$ of each input sample was transferred to $99 \mu \mathrm{DIB}+$ Proteinase $\mathrm{K}$ solution into a $1.5 \mathrm{ml}$ tube as well. From then on the input samples were treated in parallel with chromatin samples. The samples were incubated on the thermomixer at $55^{\circ} \mathrm{C}$ for $15 \mathrm{~min}$ and then at $99^{\circ} \mathrm{C}$ for an additional $15 \mathrm{~min}$. Finally, the samples were centrifuged at $14000 \mathrm{rpm}$ for $5 \mathrm{~min}$ at $4^{\circ} \mathrm{C}$ and the supernatant was collected.

qPCR analysis was performed using SYBR Green dye as described above (see "RNA Isolation and Quantitative Real Time PCR (qPCR)"). Here are the primers used:

\begin{tabular}{|l|l|l|}
\hline \multicolumn{1}{|c|}{ Primer } & \multicolumn{1}{c|}{ Sequence (5'- 3') } & Tm $^{\mathbf{}}$ \\
\hline Acot4 Promoter Forward & gctgtagcatccagggaagt & 63.2 \\
\hline Acot4 Promoter Reverse & ccacgtggtgttgtgaaagt & 63.6 \\
\hline Adcy5 Promoter Forward & gaggctctgttcgcctttc & 64.0 \\
\hline Adcy5 Promoter Reverse & cctgccagcattattttctt & 60.7 \\
\hline Ap1s3 Promoter Forward & gcgcaggtgtaagcactg & 63.4 \\
\hline Ap1s3 Promoter Reverse & tcctggcttcctcaaattgt & 63.5 \\
\hline Car4 Promoter Forward & catcttgccccaaatcaagt & 63.8 \\
\hline Car4 Promoter Reverse & cagggcttagaagcggagta & 63.3 \\
\hline Ckap4 Promoter Forward & ttcaaagcttttgcgaggat & 63.6 \\
\hline Ckap4 Promoter Reverse & ctctccacagctccagttc & 64.1 \\
\hline Dusp2 Promoter Forward & tagactccaggccgacactt & 63.8 \\
\hline Dusp2 Promoter Reverse & cgggtcaccatacaagggta & 64.5 \\
\hline E2f1 Promoter Forward & ggctctggctacgaaagaaa & 63.3 \\
\hline E2f1 Promoter Reverse & cttcaggctcacctccaaag & 63.9 \\
\hline Gabrg3 Promoter Forward & cgtgttaattggggaaactcc & 64.1 \\
\hline Gabrg3 Promoter Reverse & gctctcgggagcgatcag & 66.5 \\
\hline Gkap1 Promoter Forward & agttttaaaaatgttaatgccaatg & 60.8 \\
\hline Gkap1 Promoter Reverse & gggttggaggacagaggag & 63.8 \\
\hline
\end{tabular}




\begin{tabular}{|l|l|l|}
\hline Nkapl Promoter Forward & gctcaaggtggggaatgtaa & 63.8 \\
\hline Nkapl Promoter Reverse & cgcaggcgcactagagac & 65.1 \\
\hline Prkra Promoter Forward & tgactactgcaggcgaaaga & 63.7 \\
\hline Prkra Promoter Reverse & ggcattgtcctacgtcacaa & 63.6 \\
\hline Ptgr1 Promoter Forward & ggcctcacgacaggaagtag & 63.8 \\
\hline Ptgr1 Promoter Reverse & gaggggtgtgtgtgtgtgtt & 63.6 \\
\hline Rab38 Promoter Forward & cagcttgacaggcagtagca & 64.4 \\
\hline Rab38 Promoter Reverse & ccttcctactctgcggactc & 62.9 \\
\hline Stxbp2 Promoter Forward & gtccgttcgagtccctgtc & 64.9 \\
\hline Stxbp2 Promoter Reverse & cgtggctctacgcgtcat & 65.2 \\
\hline Syp12 Promoter Forward & gttctagtggggacccatcc & 64.4 \\
\hline Syp12 Promoter Reverse & gtcaaacaatctggcggact & 64.0 \\
\hline Tpm4 Promoter Forward & aaaggctcccaggtaagtgc & 64.3 \\
\hline Tpm4 Promoter Reverse & ctgtcaggcccatgaggt & 64.0 \\
\hline Adcy5 Exon Forward & tttctccctctgcagcttgt & 64.0 \\
\hline Adcy5 Exon Reverse & ggctcctcttcactcacctg & 64.1 \\
\hline Dusp2 Exon Forward & gacctcttggctaatcataccc & 62.5 \\
\hline Dusp2 Exon Reverse & tgcacaatgaagacaacaaattc & 63.7 \\
\hline Tpm4 Exon Forward & tctgactttccaggctgaag & 62.2 \\
\hline Tpm4 Exon Reverse & accctgtcttccacaggtc & 65.4 \\
\hline
\end{tabular}

\section{Protein Isolation and Western Blotting}

Subcellular Fractionation: Nuclear proteins were isolated with the Subcellular Fractionation Kit (Proteo Extract) from Calbiochem. The samples were dissolved in $1 \mathrm{ml}$ Extraction Buffer I with $5 \mu$ l Protease Inhibitor Mix and were incubated at $4^{\circ} \mathrm{C}$ for 10 min and after that centrifuged at $4^{\circ} \mathrm{C}$ at $1000 \mathrm{~g}$. The collected supernatant was the cytosolic fraction. The pellet was dissolved in $1 \mathrm{ml}$ Extraction Buffer II with $5 \mu \mathrm{l}$ Protease Inhibitor Mix. The samples were incubated at $4^{\circ} \mathrm{C}$ for $30 \mathrm{~min}$ and later were centrifuged at the same temperature at $6000 \mathrm{~g}$ for $10 \mathrm{~min}$. The collected supernatant was protein/organelle extract. The remaining pellet was dissolved in $500 \mu 1$ Protein Extraction Buffer III with $5 \mu 1$ Protease Inhibitor Mix and $1.5 \mu$ l Benzoase. The resulting solution was incubated at $4^{\circ} \mathrm{C}$ for $10 \mathrm{~min}$ and after that centrifuged at $10000 \mathrm{~g}$. The collected supernatant was nuclear protein extract. The remaining pellet was dissolved in $500 \mu \mathrm{l}$ Extraction Buffer with $5 \mu$ Protease Inhibitor Mix and was the cytoskeletal fraction. 
Protein Isolation with TRIZOL: For protein isolation using TRIZOL Reagent the organic phenol-chloroform phase was mixed with 120 (300) $\mu 1$ of $100 \%$ ethanol and centrifuged at $2000 \mathrm{~g}$ at $4^{\circ} \mathrm{C}$ for $5 \mathrm{~min}$. The resulting supernatant was mixed with 240 (600) $\mu \mathrm{l}$ of isopropanol and incubated at RT for $10 \mathrm{~min}$. Then the samples were centrifuged at $12000 \mathrm{~g}$ at $4^{\circ} \mathrm{C}$ for $10 \mathrm{~min}$. 400 (1000) $\mu \mathrm{l}$ of $0.3 \mathrm{M}$ guanidine hydrochloride/95\% EtOH was added to the pellet. Then the samples were incubated at RT for $20 \mathrm{~min}$ and were centrifuged for $5 \mathrm{~min}$ at $7600 \mathrm{~g}$ at $4^{\circ} \mathrm{C}$. The supernatant was discarded and $1.5 \mathrm{ml}$ of $100 \%$ EtOH was added to the pellet. The samples were incubated on a rocking shaker for 30 $\min$. Then they were centrifuged again for $5 \mathrm{~min}$ at $7600 \mathrm{~g}$ at $4{ }^{\circ} \mathrm{C}$, and were left to dry at RT for $10 \mathrm{~min}$. Finally, the pellet was dissolved in $300 \mu 1$ of $9.5 \mathrm{M}$ Urea/1\% SDS.

Protein Isolation with TX Buffer: Alternatively, proteins were also isolated by TX extraction buffer (50 mM Tris-HCl, pH 7.4; $150 \mathrm{mM} \mathrm{NaCl}$; 1mM EDTA; 1\% Nonidet P40 (IPTGAL); $0.05 \%$ SDS). A protease inhibitor tablet (Roche) was added to the extraction buffer before use and dissolved by vortexing. The tissue was homogenized in $400 \mu 1$ of extraction buffer and subjected to sonication in Bioruptor (Diagenode, Liege, Belgium) (15 min, HIGH, $30 \mathrm{sec}$ ON/OFF). The samples were incubated at $4^{\circ} \mathrm{C}$ for $5 \mathrm{~min}$ with gentle agitation. Afterwards, the samples were centrifuged at $4^{\circ} \mathrm{C}$ for $10 \mathrm{~min}$ at $14000 \mathrm{rpm}$. The supernatant was collected and used for further experiments.

Determination of Protein Concentration: Protein concentration was determined by Bradford assay (Bradford, 1976). Roti-Quant reagent (Carl Roth GmbH, Karlsruhe, Germany) was diluted 5-fold in 0.01 M PBS pH 7.0 (called Bradford Reagent henceforth). Bovine serum albumin (Sigma, Deisenhofen, Germany) was chosen to prepare the standard concentrations to generate a standard curve representing protein concentrations versus optical density. $60 \mu \mathrm{l}$ solutions of the following dilutions were prepared: $1.0,2.0,4.0,8.0,16.0,32.0$ and $64.0 \mu \mathrm{g} / \mu 1.2 \mu \mathrm{l}$ of the protein lysate and $50 \mu \mathrm{l}$ of each standard dilution were dissolved in Bradford Reagent to a total volume of 1 mlin a glass spectrophotometer cuvette $(10 \mathrm{~mm}$ x $4 \mathrm{~mm}$ x $45 \mathrm{~mm}$, Sarstedt, Nuembrecht, Germany). The Bradford Reagent was used as a blank reference. Optical density, and 
through that the protein concentration, was measured using a BioPhotometer (Eppendorf, Hamburg, Germany) at a wavelength of $595 \mathrm{~nm}$.

Alternatively, since Bradford method is not reliable at high detergent concentrations, the concentration of the protein samples isolated by TRIZOL protocol was measured by NanoDrop Spectrophotometer (PeqLab Biotechnologie GmbH, Erlangen, Germany) at a wavelength of $280 \mathrm{~nm}$.

Preparation of Protein Lysates for SDS-PAGE and Immunoblot: Protein lysates were diluted to $3 \mu \mathrm{g} / \mu \mathrm{l}$ in TX Buffer and SDS loading dye. Afterwards, the samples were incubated on a thermomixer (Eppendorf, Hamburg, Germany) at $95^{\circ} \mathrm{C}$ for $5 \mathrm{~min}$ at 1000 $\mathrm{rpm}$, and spun down shortly. The protein solution was stored at $4^{\circ} \mathrm{C}$.

SDS-Page: Proteins were resolved by SDS-PAGE (BIO-RAD Laboratories, Munich, Germany). The polymerization unit consisted of two glass plates, a spacer plate $(1.5 \mathrm{~mm})$ on the outer side and a plane plate on the inner side. The acrylamide gel consisted of a separating gel $(12 \%)$ and a stacking gel $(5 \%)$. The preparation of the gels is outlined in the table below. After the gel polymerized, the unit was put into an electrophoresis chamber which was after that filled with SDS electrophoresis buffer (25 mM Tris, 250 $\mathrm{mM}$ Glycine and $0.1 \%$ SDS in $\mathrm{ddH}_{2} \mathrm{O}$ ). $30 \mu \mathrm{g}$ of proteins were pipetted into the wells and $5 \mu$ PageRuler ${ }^{\mathrm{TM}}$ Prestained Protein Ladder was loaded into one of the wells. The proteins were resolved through a current at $60 \mathrm{~V}$ for $40 \mathrm{~min}$ and then at $120 \mathrm{~V}$ until bromophenol blue front reached the bottom of the gel.

\begin{tabular}{|l|c|c|c|}
\hline Components & 12\% Separating Gel & $6 \%$ Separating Gel & 5\% Stacking Gel \\
\hline $\mathrm{ddH}_{2} \mathrm{O}$ & $3.3 \mathrm{ml}$ & $5.3 \mathrm{ml}$ & $2.1 \mathrm{ml}$ \\
\hline $30 \%$ Acrylamide & $4.0 \mathrm{ml}$ & $2.0 \mathrm{ml}$ & $0.5 \mathrm{ml}$ \\
\hline $1.5 \mathrm{M}$ Tris $\mathrm{pH} 8.8$ & $2.5 \mathrm{ml}$ & $2.5 \mathrm{ml}$ & - \\
\hline $1.0 \mathrm{M}$ Tris $\mathrm{pH} 6.8$ & - & - & $0.38 \mathrm{ml}$ \\
\hline $10 \%$ SDS & $0.1 \mathrm{ml}$ & $0.1 \mathrm{ml}$ & $0.03 \mathrm{ml}$ \\
\hline $10 \%$ APS & $0.1 \mathrm{ml}$ & $0.1 \mathrm{ml}$ & $0.03 \mathrm{ml}$ \\
\hline TEMED & $0.004 \mathrm{ml}$ & $0.008 \mathrm{ml}$ & $0.003 \mathrm{ml}$ \\
\hline
\end{tabular}

Protein Transfer: After electrophoresis, the proteins were transferred to a nitrocellulose membrane. Transfer-Blot Cell tank transfer system (BIO-RAD Laboratories, Munich, Germany) was filled with transfer buffer (192 mM Glycine, $25 \mathrm{mM}$ Tris-HCl $\mathrm{pH} 8.3$, 
20\% Methanol) and the transfer chamber (BIO-RAD Laboratories, Munich, Germany) with the gel and the membrane attached to each other was put into it.

Immunoblot: Following transfer, the membranes were incubated in $3 \%$ milk in $0.01 \mathrm{M}$ PBS with $0.1 \%$ Triton X-100 at RT for 1 h to block non-specific binding sites. Primary antibodies were dissolved in $0.3 \%$ milk in $0.01 \mathrm{M}$ PBS with $0.1 \%$ Triton X-100. The membranes were incubated with the antibodies overnight at $4^{\circ} \mathrm{C}$. Then, the membranes were washed thrice with $0.01 \mathrm{M}$ PBS with $0.1 \%$ Triton X-100 and after that were incubated in fluorescent secondary antibody dissolved in $0.3 \%$ milk in $0.01 \mathrm{M}$ PBS with $0.1 \%$ Triton $\mathrm{X}-100$ for $2 \mathrm{~h}$ at RT in the dark. After that the membranes were washed twice with $0.01 \mathrm{M}$ PBS with $0.1 \%$ Triton X-100 and then transferred into $0.01 \mathrm{M}$ PBS and were kept in it until and throughout scanning. The membranes were scanned and the results quantified using the Odyssey Imager (Licor). The antibodies are listed in the table below:

\begin{tabular}{|c|c|c|}
\hline Primary Antibody & Manufacturer & Dilution \\
\hline Anti-Ac-H3K9 & Millipore & $1: 1000$ \\
\hline Anti-Ac-H3K14 & Millipore & $1: 1000$ \\
\hline Anti-Ac-H4K5 & Millipore & $1: 1000$ \\
\hline Anti-Ac-H4K8 & Millipore & $1: 1000$ \\
\hline Anti-Ac-H4K12 & Millipore & $1: 1000$ \\
\hline Anti-H3K4me1 & Millipore & $1: 1000$ \\
\hline Anti-H3K4me2 & Millipore & $1: 1000$ \\
\hline Anti-H3K4me3 & Millipore & $1: 1000$ \\
\hline Anti-/3-Actin & Santa-Cruz & $1: 1000$ \\
\hline & & Dilution \\
\hline Secondary Antibody & Manufacturer & $1: 15000$ \\
\hline Anti-rabbit IgG 680CW & Li-Cor & $1: 15000$ \\
\hline Anti-rabbit IgG 800CW & Li-Cor & $1: 15000$ \\
\hline Anti-mouse IgG 680CW & Li-Cor & $1: 15000$ \\
\hline Anti-mouse IgG 800CW & Li-Cor & \\
\hline
\end{tabular}

\section{Immunostaining}

Immunostainig was done on frozen sections. Brain tissue was fixed in 4\% PFA and was cut using a cryostat into $40 \mu \mathrm{m}$ sections that were stored in PBS with 
Penicillin/Streptomycin $(1: 100)$ at $4^{\circ} \mathrm{C}$. Sections were washed in $0.01 \mathrm{M}$ PBS containing $0.1 \%$ Triton $\mathrm{X}-100$. Blocking of non-specific binding sites was achieved through incubating the sections in blocking buffer $(5 \%$ goat serum $+0.3 \%$ Triton $\mathrm{X}-100$ in 0.01 M PBS) for $90 \mathrm{~min}$ at room temperature (RT). Immunostaining was performed by incubation in primary antibody diluted in blocking buffer at $4^{\circ} \mathrm{C}$ overnight. After that the sections were washed in wash buffer $(1 \%$ goat serum $+0.2 \%$ Triton X-100 in $0.01 \mathrm{M}$ PBS) thrice for $10 \mathrm{~min}$ each at RT. The sections were incubated in secondary antibody diluted in blocking buffer at RT in the dark for $2 \mathrm{~h}$. After that the sections were washed thrice for $10 \mathrm{~min}$ each in $0.01 \mathrm{M}$ PBS, and then incubated in 4',6-diamidino-2phenylindole (DAPI, $10 \mu \mathrm{g} / \mathrm{ml}$ ) for $30 \mathrm{~min}$. Finally the sections were washed twice in 0.01 M PBS at RT. The sections were then mounted on SuperFrost glass slides and dried in the dark at RT. Finally, the sections were covered with glass cover slips by adding Mowiol anti-fade medium. The sections were stored at $4{ }^{\circ} \mathrm{C}$ in the dark. The antibodies used for immunostaining are listed in the table below:

\begin{tabular}{|c|c|c|}
\hline Primary Antibody & Manufacturer & Dilution \\
\hline Anti-NeuN & Chemicon & $1: 1000$ \\
\hline Anti-MAP2 & Synaptic Systems & $1: 1000$ \\
\hline Anti-Synaptophysin & Sigma-Aldrich & $1: 1000$ \\
\hline & & Dilution \\
\hline Secondary Antibody & Manufacturer & $1: 500$ \\
\hline Cy3 anti-rabbit IgG & Jackson Immunoresearch & $1: 500$ \\
\hline Alexa488 anti-rabbit IgG & Jackson Immunoresearch & $1: 500$ \\
\hline Cy3 anti-mouse IgG & Jackson Immunoresearch & $1: 500$ \\
\hline Alexa488 anti-mouse IgG & Jackson Immunoresearch & \\
\hline
\end{tabular}

Confocal Microscope: Immunostained mouse brain sections were imaged on Leica AOBS SP2 confocal microscope (Leica Microsystems, Wetzlar, Germany). Alexa488 and Cy3 conjugated secondary antibodies were excited using $488 \mathrm{~nm}$ and $561 \mathrm{~nm}$ lasers respectively. 


\section{Statistical Analysis}

Data were analysed by unpaired Student's t-test and two-way ANOVA (Analysis of Variance) when appropriate. Errors are displayed as standard error of mean (SEM). Graphs were generated using GraphPad Prism. 


\section{RESULTS:}

\section{PART 1: CHARACATERIZATION OF FOREBRAIN SPECIFIC MLL2 CONDITIONAL KNOC-OUT MICE}

\section{Mll2 Expression in Different Brain Regions}

First, I analyzed the endogenous levels of Mll2 expression in different regions of the brain with qRT-PCR. Interestingly, I observed that Mll2 expression is slightly higher in dentate gyrus (DG) compared to CA region and prefrontal cortex (PFC) (Figure 1.1). Though, the difference failed to reach significance.

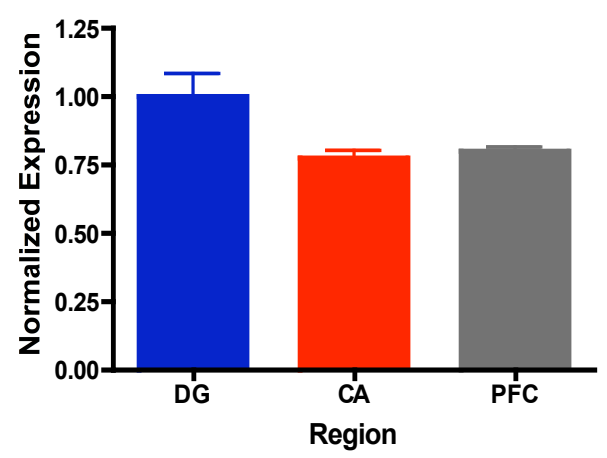

Figure 1.1: Mll2 mRNA expression in different brain regions $(n=4)$.

\section{Creating forebrain specific MII2 conditional knock-out mice}

The mice heterozygous for the floxed exon 2 of Mll2 were a kind gift of the group of Francis Stewart and Andrea Kranz (Biotechnology Centre - Technical University Dresden). They were crossed to the mice bearing a Cre transgene under a CamKII promoter in order to confer forebrain specificity for Cre expression. The heterozygous mice bearing the Cre transgene that originated from this cross were then crossed to each other in order to give rise to homozygous floxed mice without the Cre transgene (F/F) and homozygous floxed mice with the Cre transgene (F/F CKII) which were the conditional knock-out mice. The F/F mice were used as controls in all the experiments 
that followed. In the homozygous mice bearing the Cre transgene under CamKII promoter $2^{\text {nd }}$ exon of Mll2 was recombined. The presence of FRT sequence between the $1^{\text {st }}$ and $2^{\text {nd }}$ exon ensured that this recombination resulted in a premature stop codon through frameshift mutation. The scheme used for obtaining the conditional knock-out is outlined in the figure below.

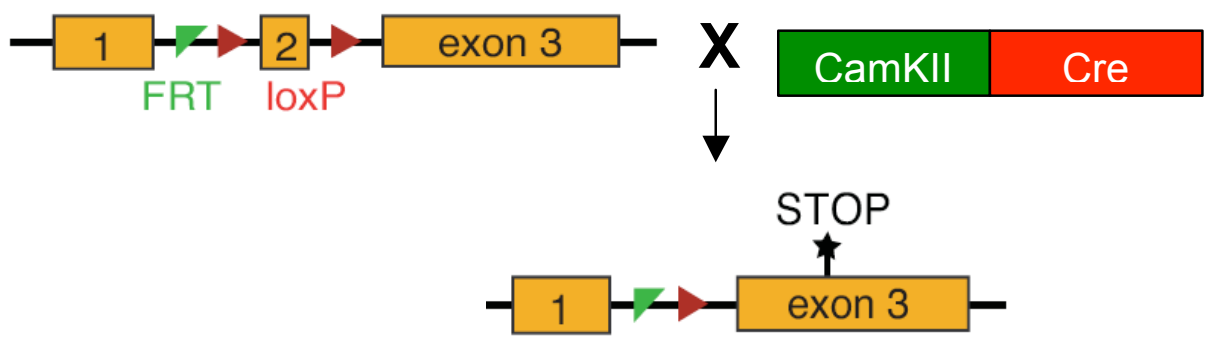

Figure 1.2: The exon 2 of Mll2 gene is contains loxP sites at its borders. The removal of exon 2 through Cre recombination results in a frameshift mutation which induces a premature stop codon.

The expression of the full-length transcript of $M I l 2$ is decreased in the forebrain of F/F CKII mice

Next, I compared the expression of the full-length transcript of Mll2 in DG, CA, prefrontal cortex and cerebellum in F/F and F/F CKII mice. For this purpose I used a primer pair targeting exon 1 and exon 2 of Mll2 gene. As expected, I saw a downregulation of the full-length transcript of Mll2 in the hippocampus, prefrontal cortex but not in the cerebellum of the CKII mice (Figure 1.3A).
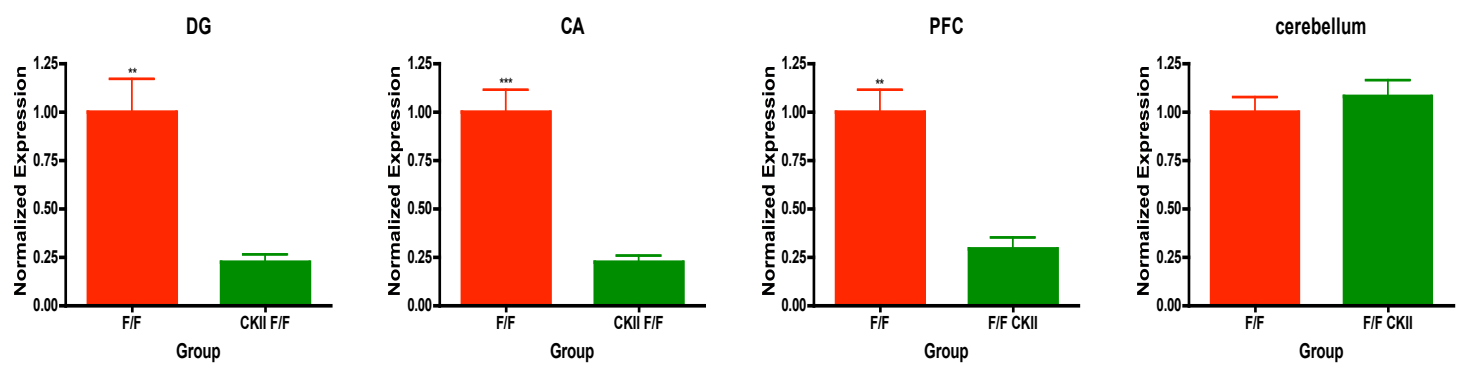

Figure 1.3: Recombination of exon 2 in the Mll2 gene occurs in hippocampus (DG and CA) and prefrontal cortex but not in cerebellum. $(\mathrm{n}=9, * * p<0.01, * * * p<0.001)$. 
Moreover, the knock-down of M112 in the forebrain does not lead to any change in M111 and Ml13 - its close homologs (Figure 1.4).
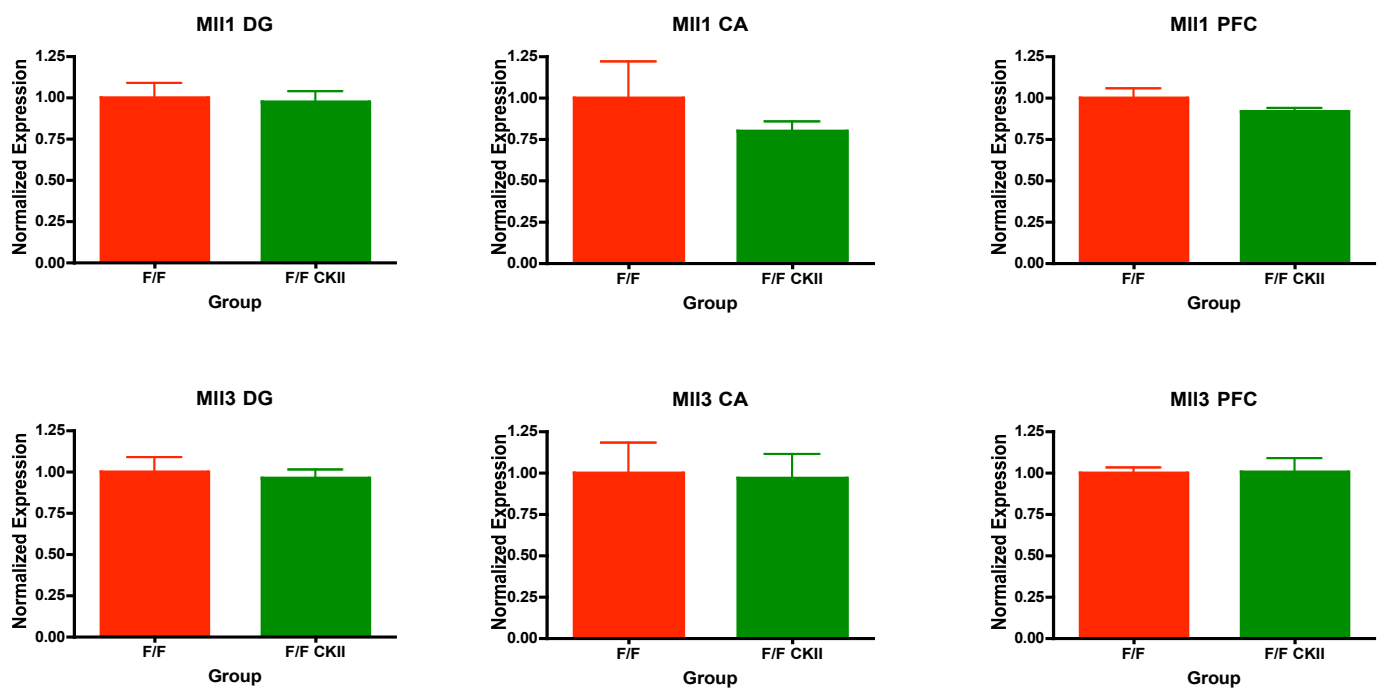

Figure 1.4: There is no up or down regulation of Mll1 or Mll3 in response to Mll2 knock-down $(\mathrm{n}=5)$.

\section{Brain morphology and body size are normal in F/F CKII mice}

Brain and body size of F/F CKII mice were comparable to those of the controls (Figure 1.5). Although female F/F CKII mice showed a slightly increased body weight compared to their control counterparts (Figure 1.5).
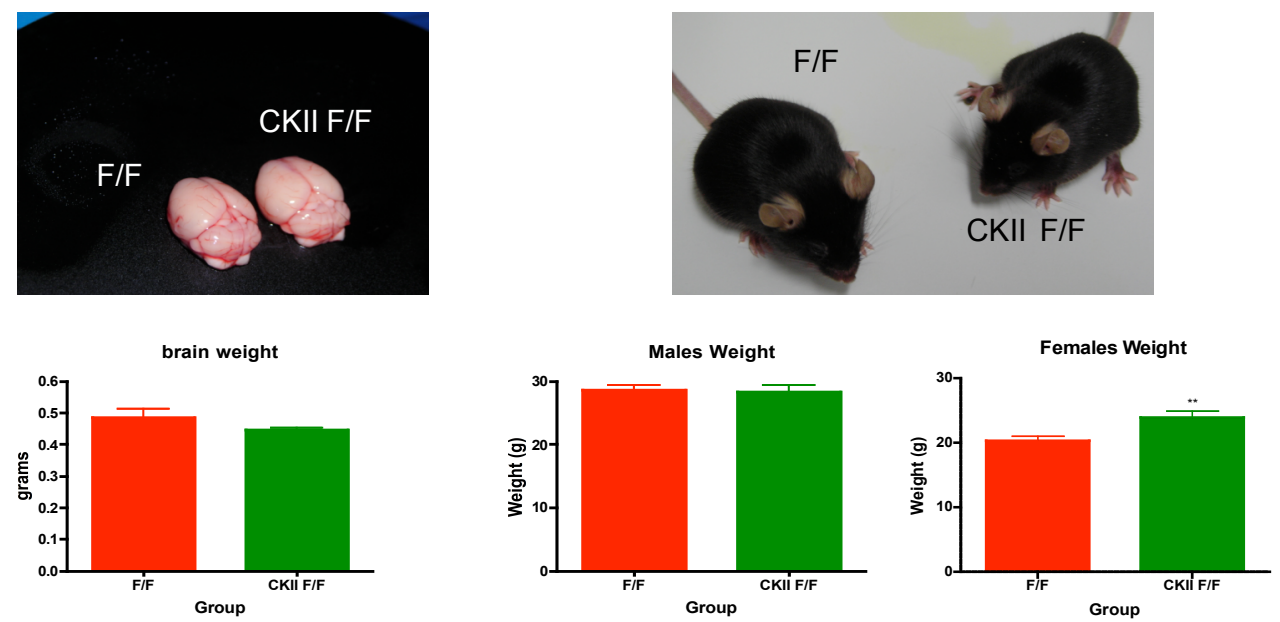

Figure 1.5: The brain $(n=8)$ and body weight $(n=10)$ of F/F CKII mice do not differ from those of the controls. Although female knock-out mice show a slightly increased body weight $(p<0.01)$. 
Next, the neuronal and synaptic integrity was tested in F/F CKII mice. Staining for MAP2 indicates that there is no difference in dendrite density between F/F and CKII

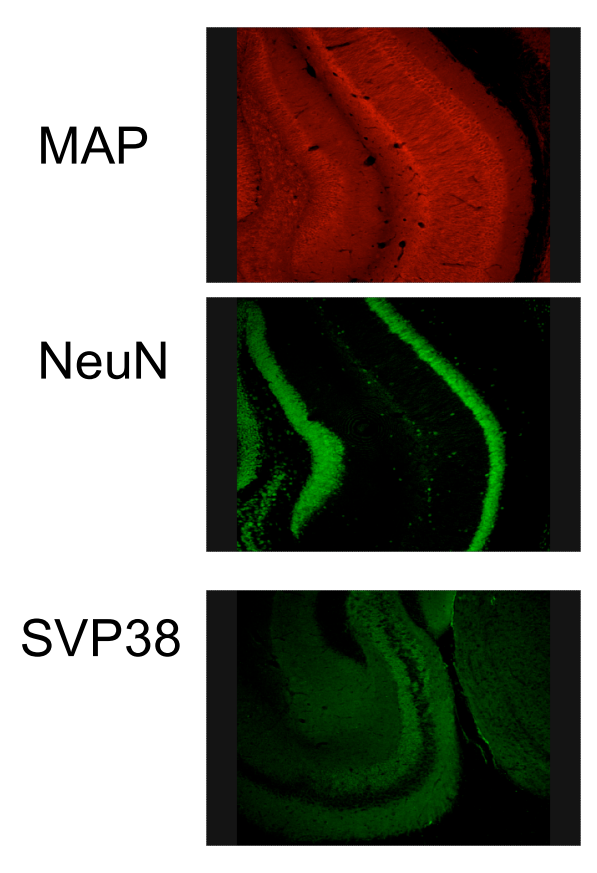

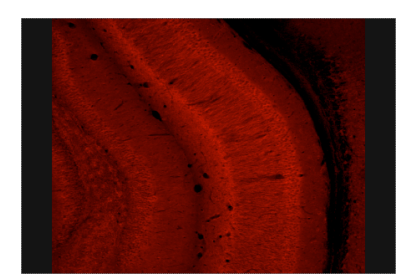
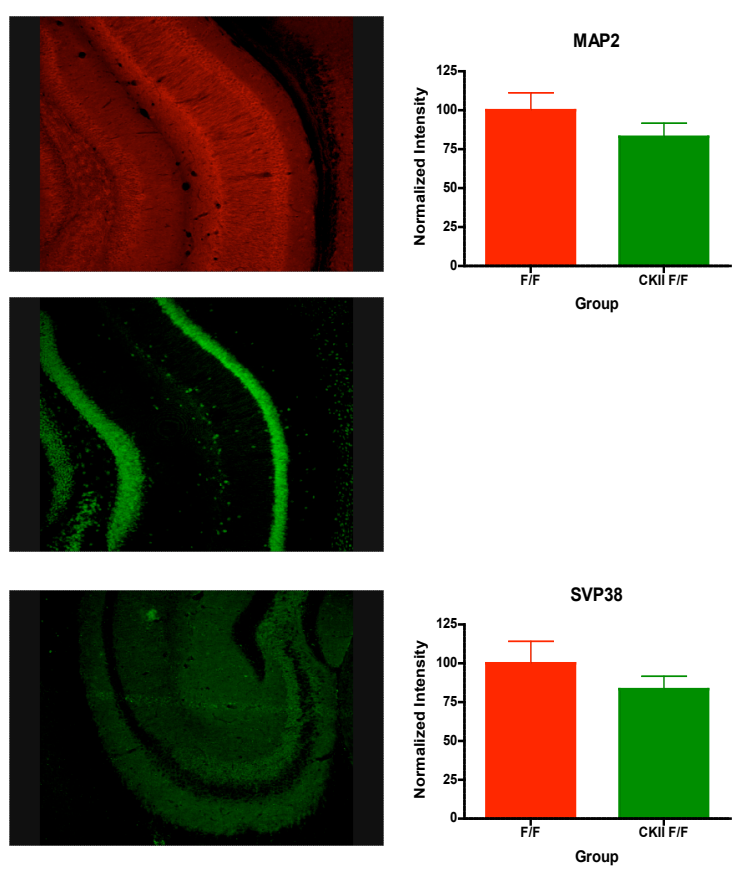

Figure 1.6: F/F CKII mice have a normal neuronal and synaptic morphology as observed by MAP2, NeuN and Synaptophysin staining $(n=4)$.

F/F mice. Synaptophysin staining showed an uncompromised integrity of the presynaptic terminals in F/F CKII mice. And staining for NeuN did not reveal any obvious differences between groups (Figure 1.6).

\section{Knock-down of MIl2 in the forebrain leads to increased anxiety and learning deficits}

It has previously been shown that Mll1 heterozygous knock-out mice show impaired synaptic plasticity and memory impairment in fear conditioning (Kim et al., 2007; Gupta et al., 2010). Therefore I set out to study the effect of Ml12 knock-out in the forebrain on behaviour in mice. 
Increased Anxiety: Firstly, I observed that F/F CKII mice show increased anxiety compared to the controls. This manifested itself in significantly decreased time spent in the center of an open field and in the open arms of an elevated plus maze (Figure 1.7A). However, total distance travelled in the open field and total object exploration did not differ between F/F and F/F CKII mice (Figure 1.7B).

A.

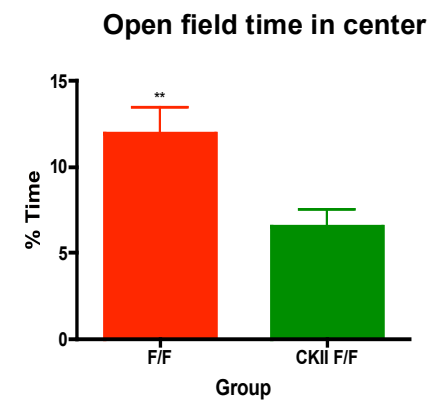

B.

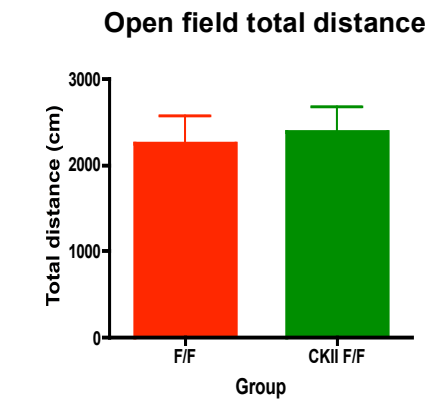

Elevated plus maze (open arms)

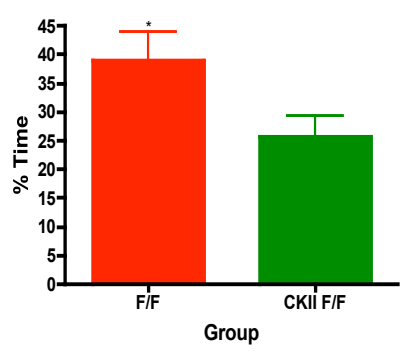

Figure 1.7: (A) M112 knock-down in the forebrain leads to increased anxiety as measured by Open Field (F/F: $n=30$; F/F CKII: $\mathrm{n}=31)$ and Elevated Plus Maze paradigms (F/F: $\mathrm{n}=19 ; \mathrm{F} / \mathrm{F} \mathrm{CKII} \mathrm{n}=26)(* p<0.05, * * p<0.01)$.

(B) M112 knock-down does not lead to a change in total activity (F/F: $\mathrm{n}=24 ; \mathrm{F} / \mathrm{F}$ CKII: $\mathrm{n}=26)$.

Learning Impairment: F/F CKII mice also showed impaired performance in diverse learning paradigms - i.e. novel object recognition (NOR), Pavlovian fear conditioning and Morris water maze (Figure 1.8). This reflected itself in no preference for the new object above $50 \%$ chance level in novel object recognition paradigm, both $5 \mathrm{~min}$ and $24 \mathrm{~h}$ after exposure to similar objects (Figure 1.8A); in significantly lower freezing upon reexposure to the same context $24 \mathrm{~h}$ after receiving the footshock (Figure 1.8B); and in no preference to the target quadrant above chance level in the probe test of Morris water maze task (Figure 1.8C). Interestingly however, when mice were subjected to a more 
A. NOR short-term memory

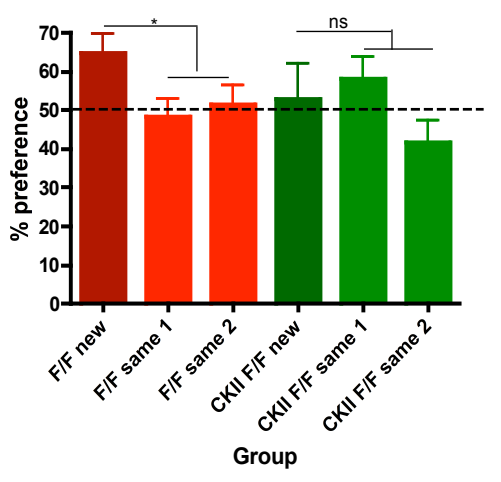

B.

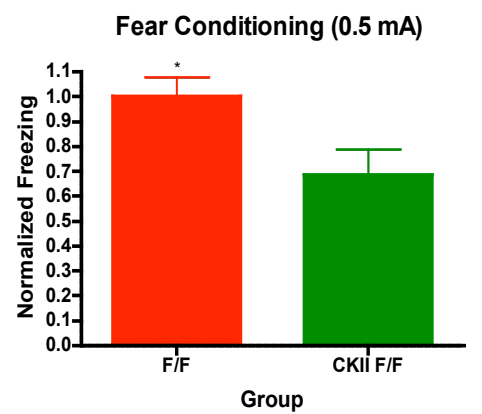

C.

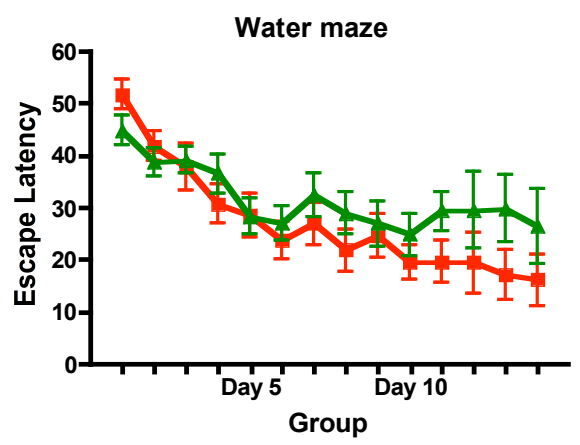

NOR long-term memory

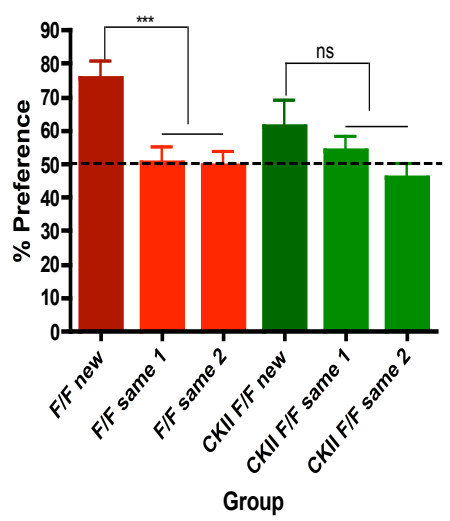

Fear Conditioning $(0.7 \mathrm{~mA})$

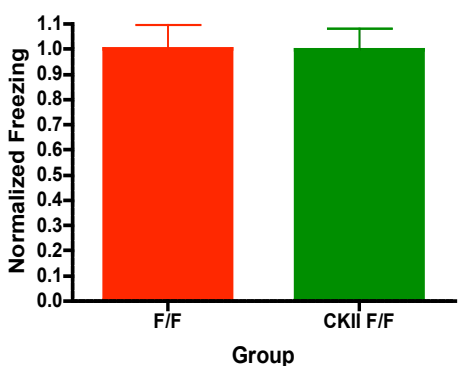

$\rightarrow F / F$ $\rightarrow$ CKII F/F

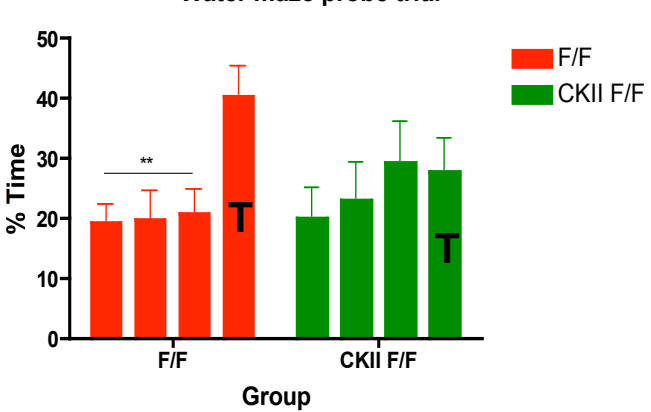

Figure 1.8: (A) Ml12 knock-down leads to a deficiency in short-term and long-term object memory manifested in F/F CKII mice showing no preference to a new object over the old one (F/F: $\mathrm{n}=14 ; \mathrm{F} / \mathrm{F}$ CKII: $\mathrm{n}=10 ;{ }^{*} p<0.05, * * * p<$ $0.001)$.

(B) Ml12 F/F CKII mice display a lower freezing level upon reexposure to the context $24 \mathrm{~h}$ after the $0.5 \mathrm{~mA}$ footshock ( $F / F: n=20 ; F / F$ CKII: $n=15)$, however their freezing level is comparable to that of controls if $0.7 \mathrm{~mA}$ footshock is applied (F/F: $\mathrm{n}=22 ; \mathrm{F} / \mathrm{F}$ CKII: $\mathrm{n}=25 ; * p<0.05)$.

(C). M112 F/F CKII mice show and impaired spatial memory in Morris Water Maze task with no preference to the target quadrant during probe trial (F/F: $\mathrm{n}=19 ; \mathrm{F} / \mathrm{F} \mathrm{CKII:} \mathrm{n}=23 ; * * p<0.01)$. 
stringent protocol of the fear conditioning paradigm ( $0.7 \mathrm{~mA}$ footshock) the F/F CKII mice did not show any impairment.

In all of the behavioural graphs shown (except for novel object recognition test) males and females were grouped together. No sex specific effects and no sex-genotype interaction with respect to behavioural performance were revealed by two-way ANOVA. Only male mice were included in the graphs showing the performance in novel object recognition task; the reason for this was that females of both groups barely approached the objects during the training session. There was however no difference between the genotypes in total object exploration time during testing (data not shown).

These results clearly indicate that Mll2 functioning is crucial for a wide range of memory types (object recognition memory, associative memory, spatial memory) and anxiety behaviour.

\section{Confirmation of differences in dorso-ventral gene expression pattern in hippocampus}

There exists a dorso-ventral specificity of function within hippocampus, with dorsal hippocampus responsible for learning \& memory (esp. spatial memory) and ventral hippocampus involved in anxiety (Moser and Moser, 1998). And since Mll2 F/F CKII mice show increased anxiety together with learning impairment it was important to study dorsal and ventral parts of hippocampus separately in molecular terms in order to dissociate involvement of M112 in anxiety phenotype from its involvement in learning \& memory.

I decided to analyse gene expression in dorsal and ventral hippocampus individually. In order to confirm that our dissection method is reliable I selected some genes, which have previously been shown to have dorso-ventral specificity in hippocampus (Thompson et al., 2008; Dong et al., 2009; Fanselow and Dong, 2010), and compared their relative expression in dorsal and ventral hippocampus by qRT-PCR. I observed that genes, which were shown to be dorsal specific, were expressed at a higher level in dorsal hippocampal samples, and those known to be ventral specific had a higher expression in ventral hippocampal samples (Figure 1.9). 

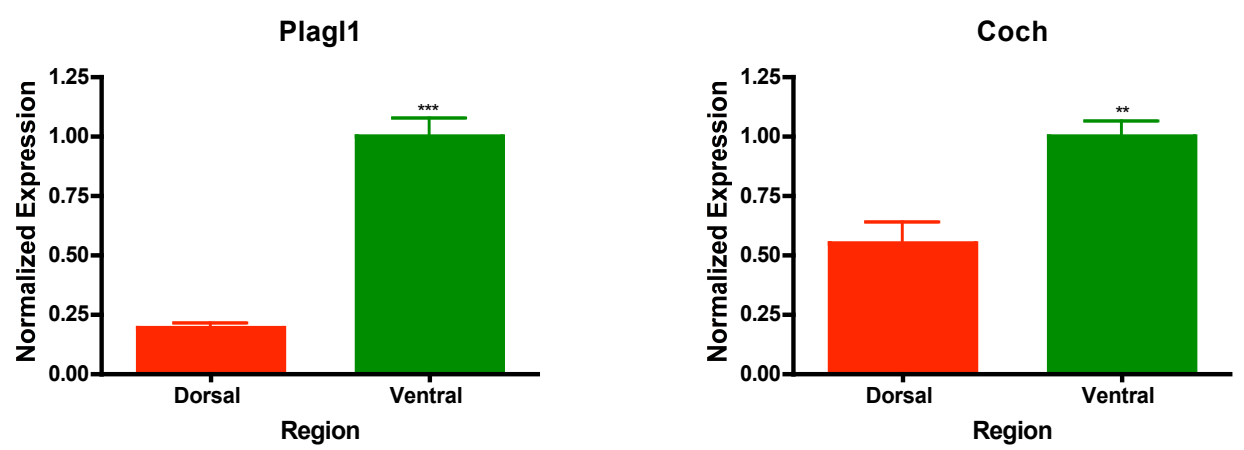

Wfs1

Fmo1
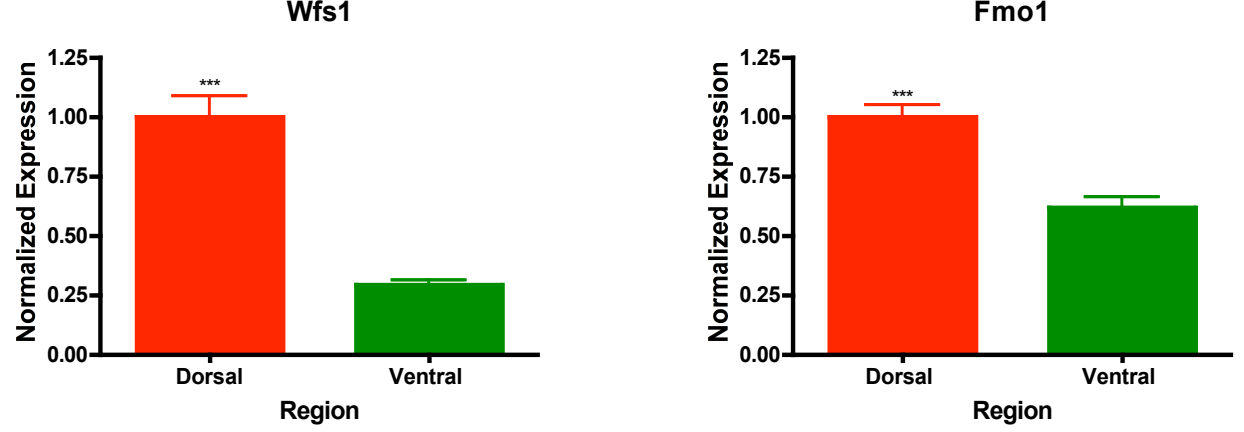

Figure 1.9: Differential expression of genes showing dorso-ventral specificity was confirmed by qRT-PCR in ventral (A) and in dorsal (B) hippocampus $(\mathrm{n}=4, * * p<0.01, * * * p<0.001)$.

\section{Knock-down of MIl2 leads to deregulation of gene expression in dentate gyrus}

The next thing I wondered was whether M112 knock-down leads to changes in gene expression profile that could lead to the observed learning impairment. For this purpose a DNA microarray analysis was carried out from dorsal dentate gyrus and dorsal CA regions of $\mathrm{F} / \mathrm{F}(\mathrm{n}=3)$ and $\mathrm{F} / \mathrm{F} \mathrm{CKII}(\mathrm{n}=5)$ mice. It was observed that knock-down of Mll2 lead to a significant reduction in expression levels of 153 genes (fold change $=1.5$; FDR $=0.05$ ) in the dorsal dentate gyrus (Figure 1.10) which is consistent with the fact that M112, as a histone 3 methyltransferase, is a transcriptional activator (Demers et al., 2007; Glaser et al., 2009). Moreover, there were also 10 genes, which were upregulated the dorsal dentate gyrus of F/F CKII mice, which may be attributed to secondary effects of M112 knock-down (see the full list of differentially regulated genes in Appendix 1). 16 of the downregulated genes were chosen for further qPCR confirmation (Figure 1.11). 
Interestingly however, such a drastic downregulation of gene expression was not observed in the dorsal CA region (Figure 1.10).

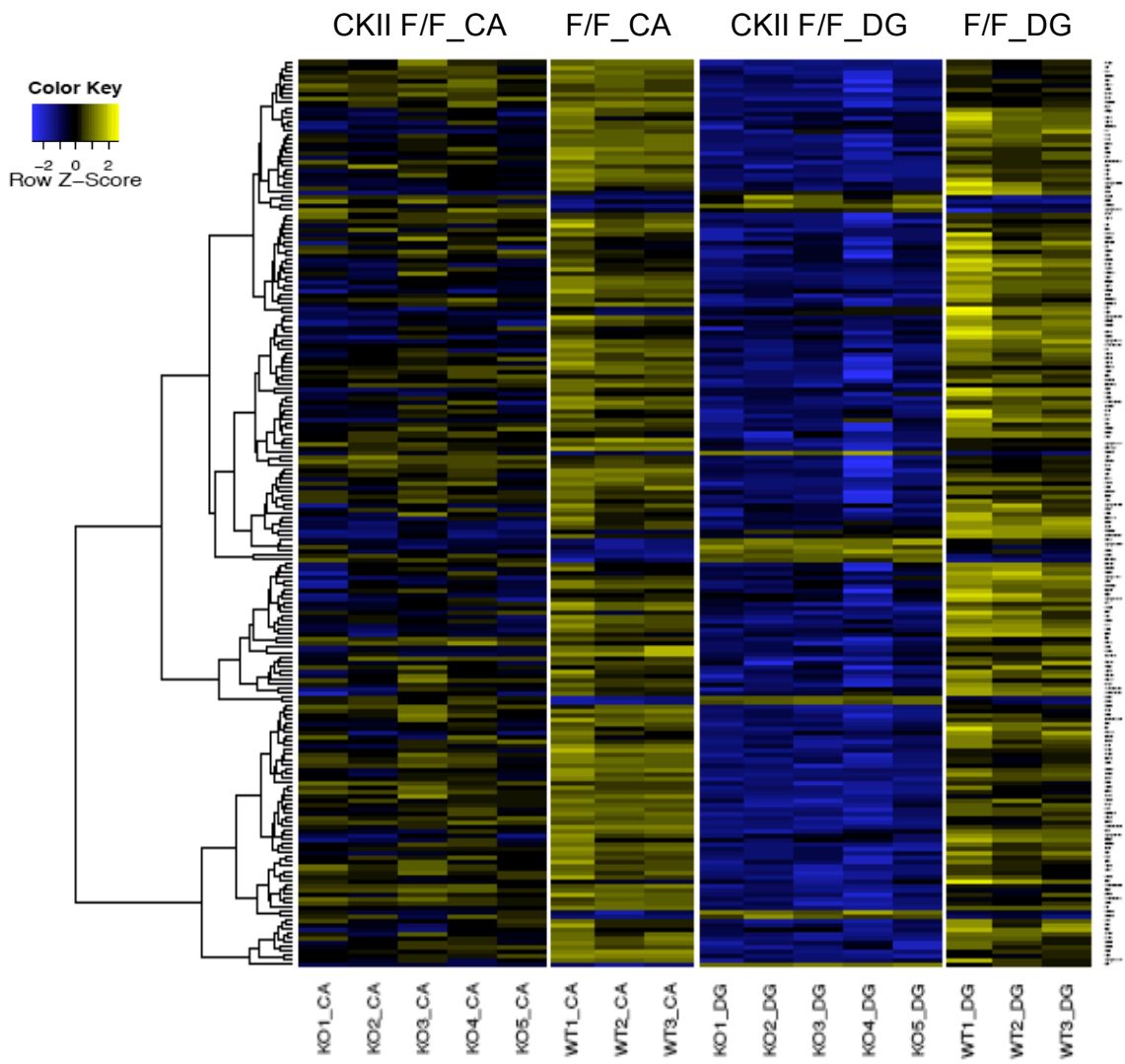

Dorsal DG

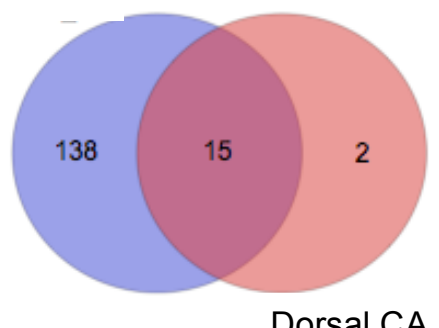

Figure 1.10: M1l2 knock-down leads to extensive downregulation of gene expression in dorsal dentate gyrus but not in dorsal CA (F/F: $n=3$; F/F CKII: $n=5)$. The Venn diagram shows the number of downregulated genes in DG and CA regions of M112 F/F CKII mice. 

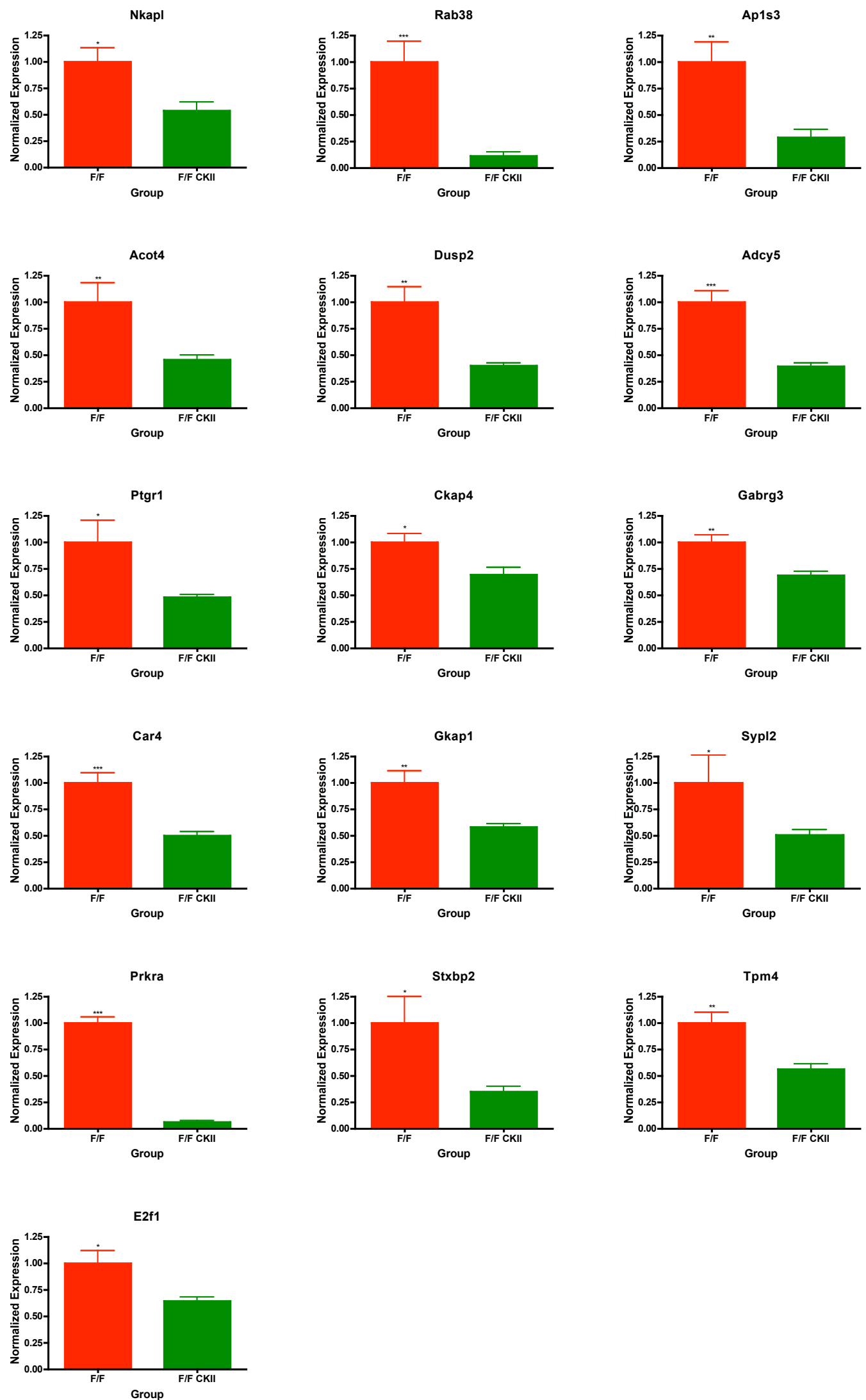

Figure 1.11: 16 genes that were found to be donwregulated in dorsal dentate gyrus of $\mathrm{F} / \mathrm{F}$ CKII mice were chosen for qRT-PCR confirmation. All of them were confirmed (F/F: $\mathrm{n}=3$; F/F CKII: $\mathrm{n}=5 ; * p<0.05, * * p<0.01, * * * p<0.001$ ). 

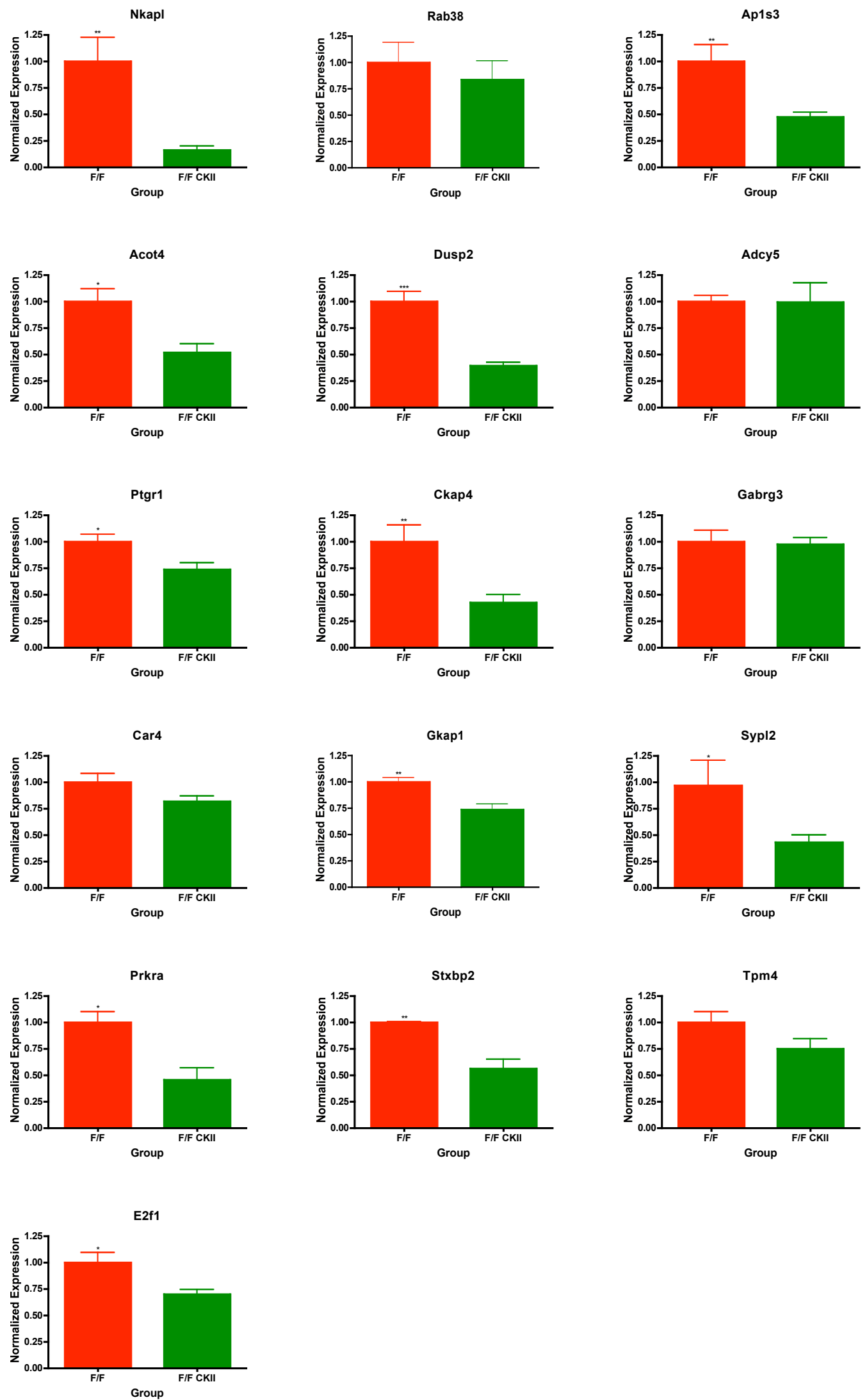

Figure 1.12: Most of the genes that were downregulated in dorsal dentate gyrus in F/F CKII mice are also downregulated in the ventral dentate gyrus. However, in spite of being downregulated in dorsal dentate gyrus, Rab38, Adcy5, Gabrg3, Car4 and Tpm4 do not show downregulation in ventral part (F/F: $\mathrm{n}=3$; F/F CKII: $\mathrm{n}=5 ; * p<0.05, * * p<0.01, * * * p<$ $0.001)$. 

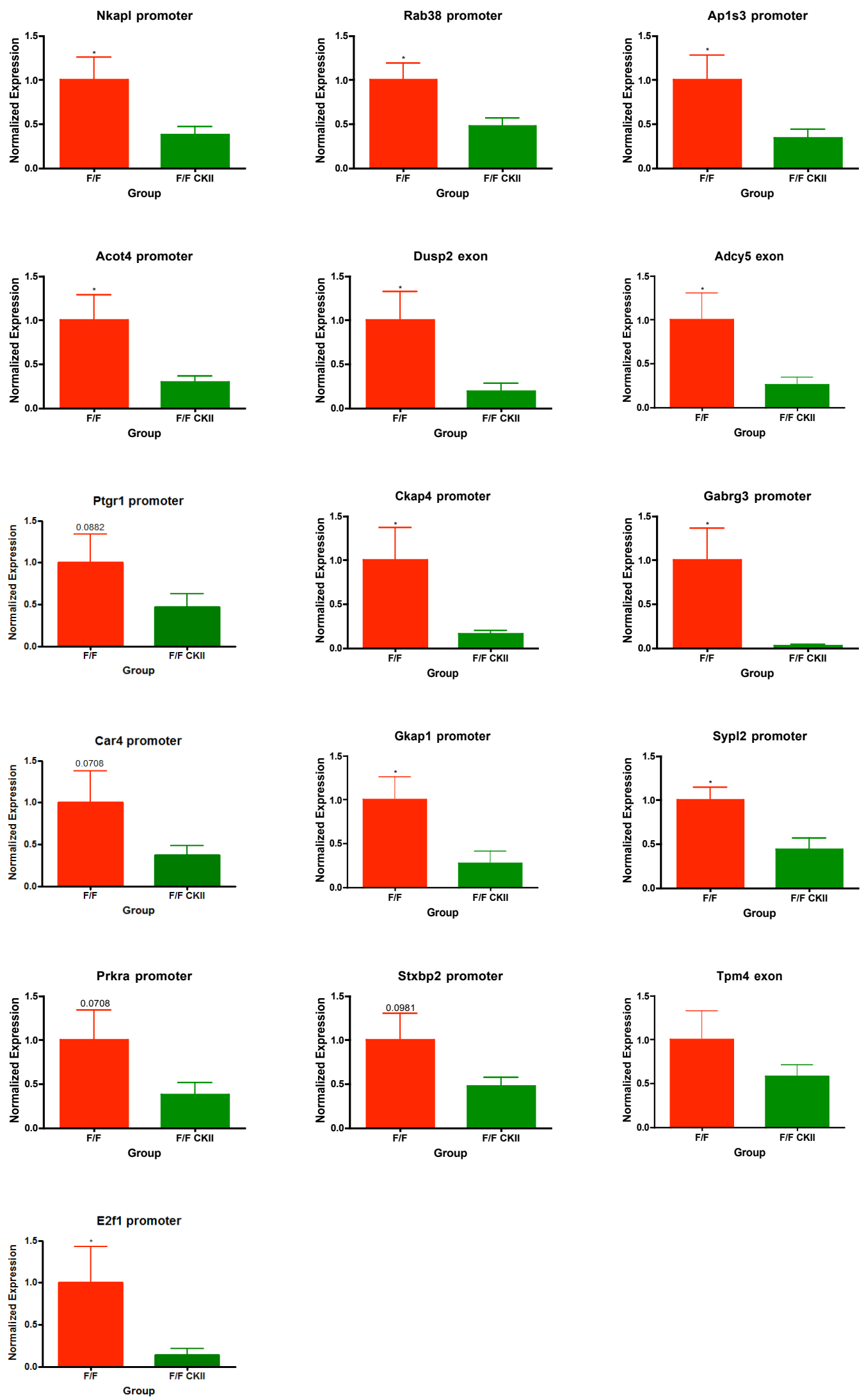

Figure 1.13: The genes that are downregulated in the dorsal dentate gyrus of F/F CKII mice also show decreased level of H3K4 trimethylation at their promoters $(\mathrm{n}=5 ; * p<0.05)$. 
The genes chosen for qPCR confirmation were also reconfirmed in a separate batch of mice, which were not used for the microarray. Interestingly, 4 of those genes (Rab38, Adcy5, Gabrg3, Car4, Tpm4), which were downregulated in the dorsal dentate gyrus, were not downregulated in the ventral dentate gyrus of the knock-out mice (Figure 12), which indicates that there is some dorso-ventral specificity of M112 action in dentate gyrus.

\section{MIl2 knock-down leads to decreased histone 3 Lysine 4 methylation (H3K4me) at gene promoters}

Since there was an extensive downregulation of gene expression in dentate gyrus as a result of M112 knock-down, my next step was to study the level of H3K4 methylation at the promoters of those genes. In line with the findings from the DNA microarray ChIP analysis revealed downregulation in $\mathrm{H} 3 \mathrm{~K} 4 \mathrm{me} 3$ (Figure 1.13) and $\mathrm{H} 3 \mathrm{~K} 4 \mathrm{me} 2$ (Figure 1.14), but not in $\mathrm{H} 3 \mathrm{~K} 4 \mathrm{me} 1$ levels (Figure 1.15) at the gene promoters (primers for the promoters of Dusp2, Adcy5 and Tpm4 did not lead to an amplification, therefore primers targeting the coding region were used for these genes). At some gene promoters (i.e. Ptgr1, Car4, Prkra, Stxbp2) H3K4me3 levels showed a strong tendency towards downregulation in the F/F CKII mice, which nevertheless failed to reach significance. These results further confirm that histone methyltransferase activity of M112 plays an important role in regulating gene expression in the dentate gyrus, and through this in affecting the cognitive and behavioural phenotype in mice.

\section{Injection of HDAC inhibitor SAHA does not change the expression of the genes neither in $\mathrm{F} / \mathrm{F}$ nor in $\mathrm{F} / \mathrm{F}$ CKII mice}

It has been indicated in a previous study that the Histone 3 Lysine 4 methyl mark serves as a prerequisite for the binding of histone acetyltransferases (HATs) at the promoter regions (Wang et al., 2009); in other words Histone 3 Lysine 4 methylation serves a key to enable histone acetylation at the same promoter. It has already shown in our lab that 

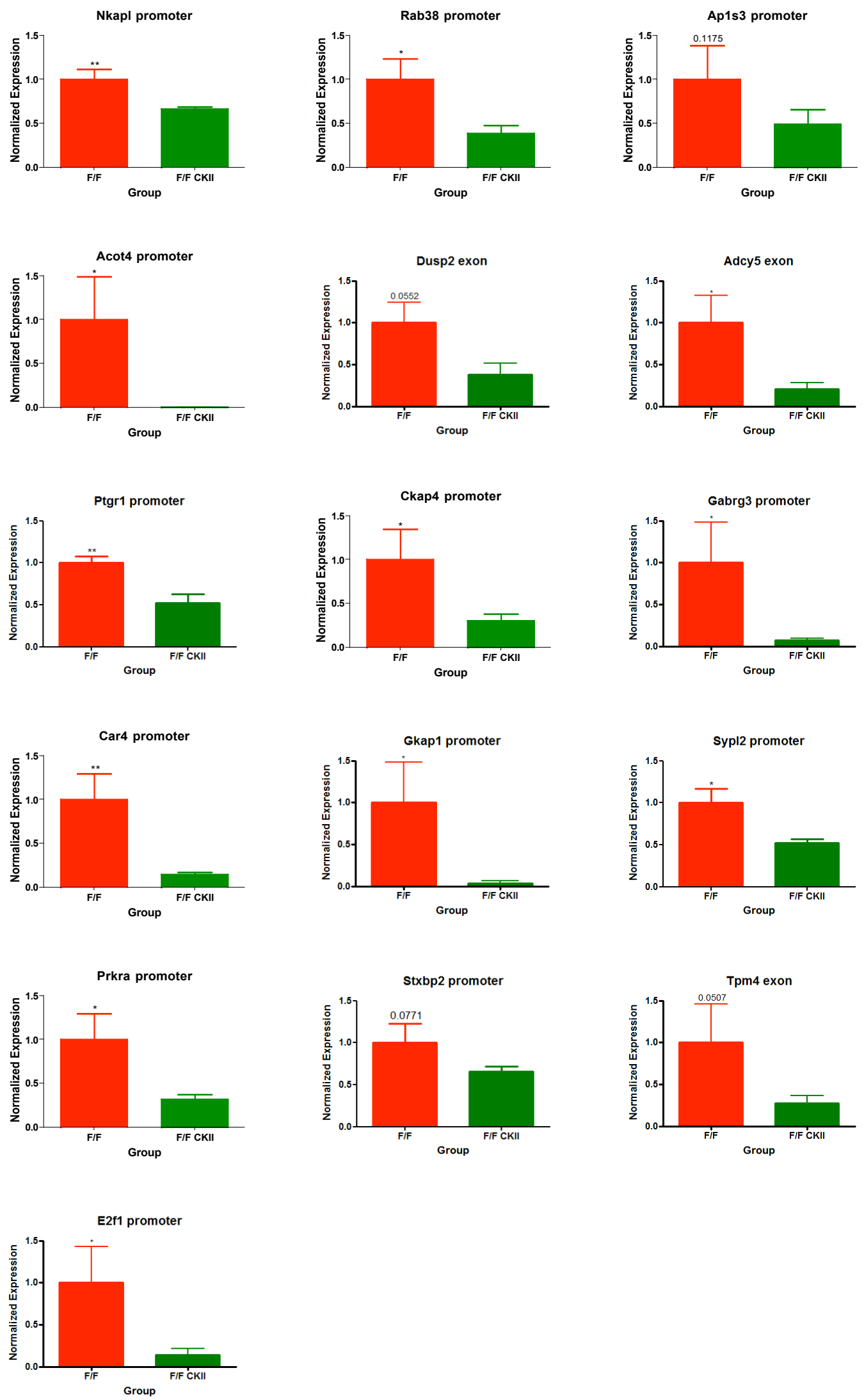

Figure 1.14: The genes that are downregulated in the dorsal dentate gyrus of $F / F$ CKII mice also show decreased level of H3K4 dimethylation at their promoters $\left(\mathrm{n}=5 ;{ }^{*} p<0.05, * * p<0.01\right)$. 

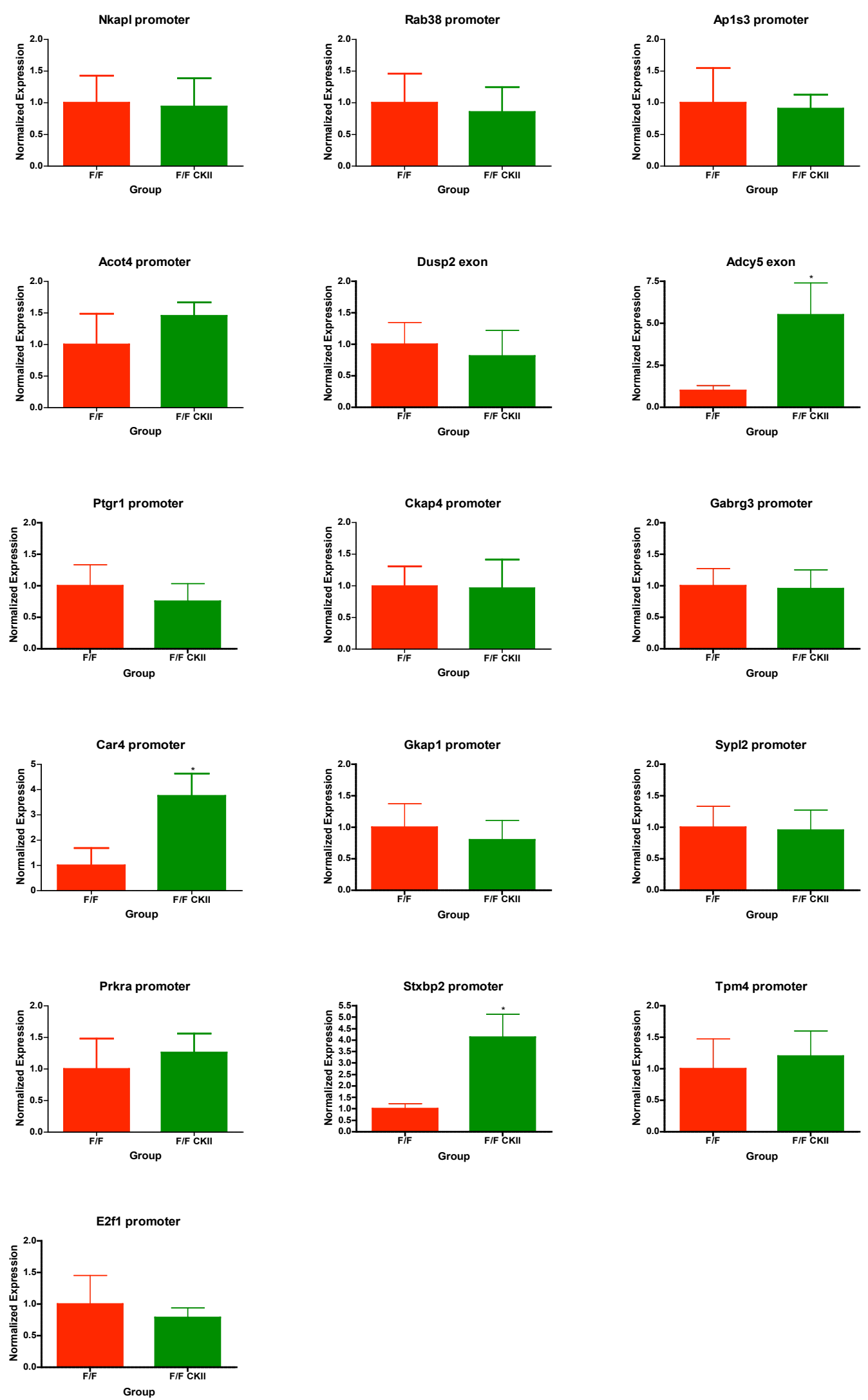

Figure 1.15: The genes that are downregulated in the dorsal dentate gyrus of F/F CKII mice do not show a decrease in the level of H3K4 monomethylation at their promoters $\left(\mathrm{n}=5 ;{ }^{*} p<0.05\right)$. 
acute intraperitoneal injection of SAHA leads to an increase in bulk histone acetylation in hippocampus of wild type B6J mice, which peaks at 1 hour after injection. Therefore, before proceeding with the analysis of gene expression I tested the effect of SAHA in hippocampus of F/F and F/F CKII mice 1 hour after injection. Both F/F and F/F CKII mice had an increase in bulk histone acetylation 1 hour after SAHA treatment (Figure 1.16). Next, I set out to investigate whether treatment of mice with an HDAC inhibitor would increase the expression of the genes, which were downregulated in F/F CKII mice. For this purpose another group of $\mathrm{F} / \mathrm{F}$ and $\mathrm{CKII} \mathrm{F} / \mathrm{F}$ mice was injected intraperitoneally either with SAHA or vehicle. The expression of selected genes was tested in the dorsal dentate gyrus of $\mathrm{F} / \mathrm{F}$ and $\mathrm{F} / \mathrm{F}$ CKII mice $1 \mathrm{~h}$ after they received the intraperitoneal SAHA injection. Interestingly however, it was observed that SAHA injection did not lead to an increase in the expression of the genes previously shown to be downregulated as a result of Mll2 knock-down, neither in F/F nor in F/F CKII mice (Figure 1.17).

A.
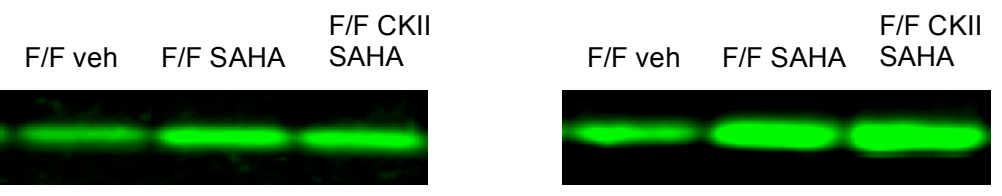

$\mathrm{H} 4 \mathrm{~K} 8 \mathrm{ac}$

$\mathrm{H} 3 \mathrm{~K} 9 \mathrm{ac}$

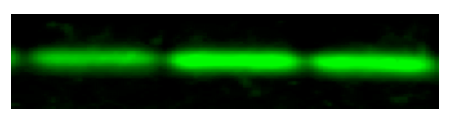

(1)
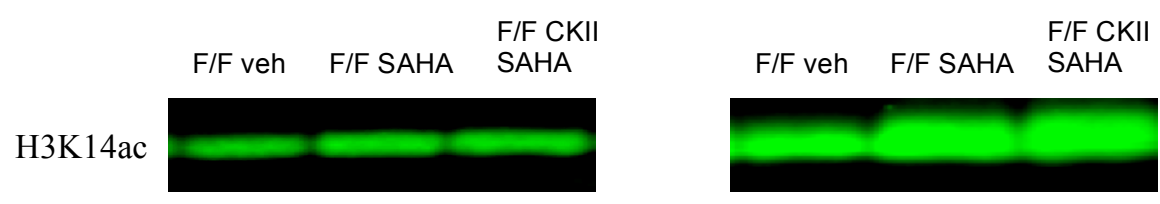

$\mathrm{H} 4 \mathrm{~K} 12 \mathrm{ac}$

B.
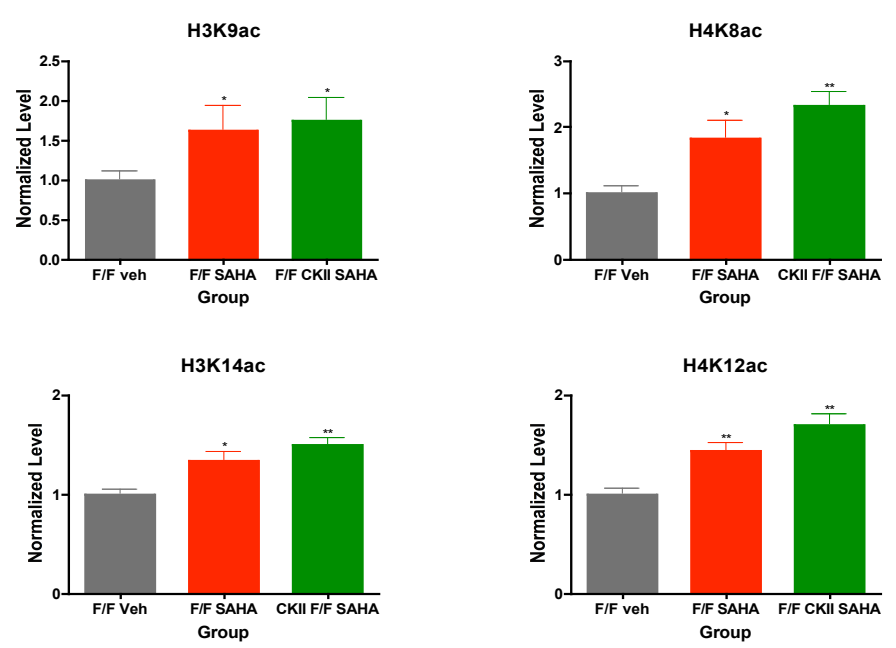

Figure 1.16: (A) Acute SAHA injection increases bulk histone acetylation in hippocampus of $\mathrm{F} / \mathrm{F}$ and $\mathrm{F} / \mathrm{F}$ CKII mice 1 hour after injection.

(B) Quantification of the immunoblots in (A) (F/F veh: $\mathrm{n}=4$; F/F SAHA: $\mathrm{n}=5$; F/F CKII SAHA: $\mathrm{n}=5$; $* p<0.05, * * p<0.01)$. 

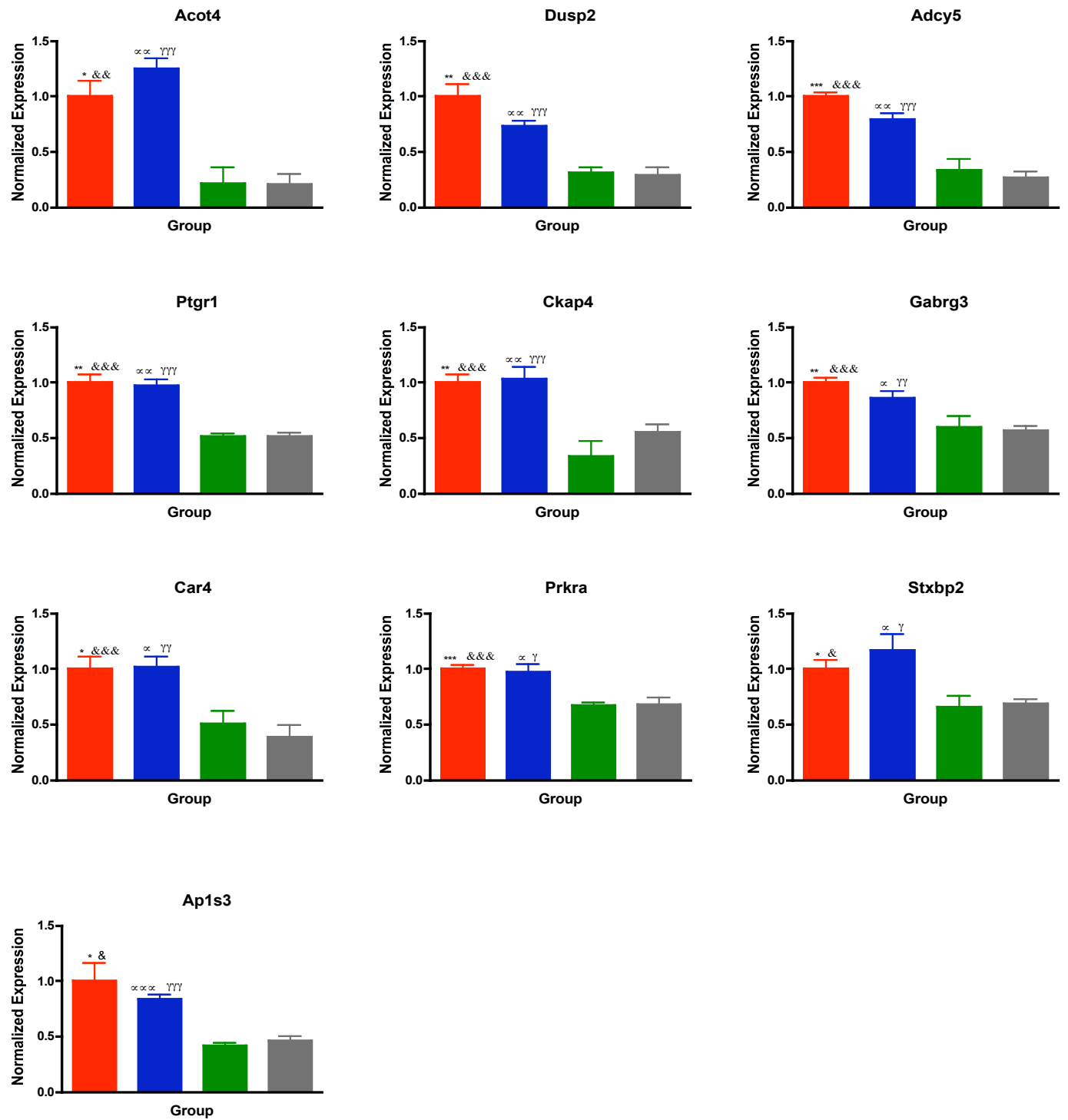

Figure 1.17: The genes found to be downregulated after Mll2 knock-down do not show any change in expression after SAHA injection in either group $(*=\mathrm{F} / \mathrm{F}$ Vehicle vs CKII F/F Vehicle; $\&=\mathrm{F} / \mathrm{F}$ Vehicle vs CKII F/F SAHA; $\alpha=\mathrm{F} / \mathrm{F}$ SAHA vs CKII F/F Vehicle; $\gamma=$ F/F SAHA vs CKII F/F SAHA; *, \&, $\alpha, \gamma p<0.05 ; * *, \& \&, \alpha \alpha, \gamma \gamma p<0.01 ; * * *, \& \& \&$, $\alpha \alpha \alpha, \gamma \gamma \gamma p<0.001)$. 


\section{PART 2: EFFECTS OF DIFFERENT DURATIONS OF ENVIRONMENTAL ENRICHMENT ON LEARNING AND MEMORY}

\section{2 weeks of EE are enough to facilitate learning whereas 1 week of EE is not}

My first aim was to establish the minimum amount of EE in our experimental setting, which would be enough to facilitate learning. Up to this day there is no standardized environmental enrichment protocol. That is why before proceeding with further experiments it was necessary to establish what amount of EE is enough to facilitate learning in our laboratory. I observed that 2 weeks of EE were enough to facilitate learning in Pavlovian fear conditioning paradigm, whereas 1 week of EE was not (Figure 2.1). This finding obviously does not exclude the possibility that environmental enrichment performed for an amount of time between $1-2$ weeks will also be enough to facilitate learning, however for the sake simplicity in all further experiments I performed environmental enrichment for at least 2 weeks. And for the sake of simplicity in presentation and discussion of results I will treat 2 weeks of EE as the minimum amount of EE required to facilitate learning henceforth.

A.

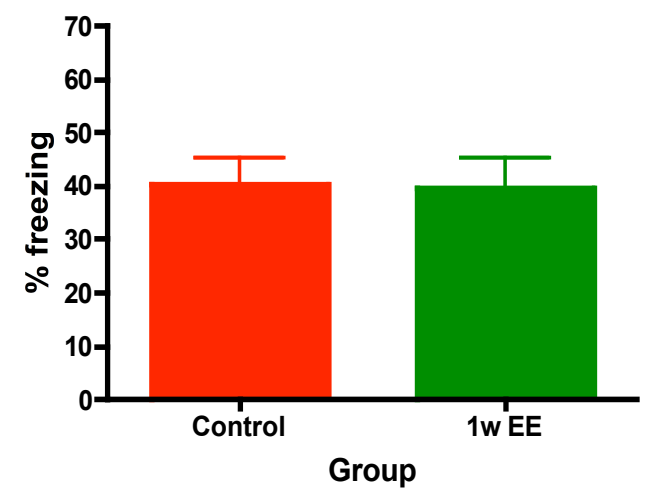

B.

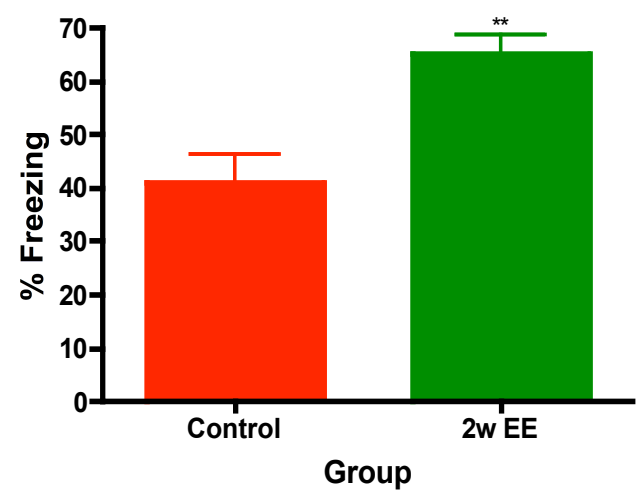

Figure 2.1: Environmental enrichment of a duration of 1 week is not enough to facilitate learning (A) in wild-type B6J mice, whereas it does facilitate learning when performed for 2 weeks (B) $(\mathrm{n}=8 ; p<0.01)$. 


\section{Long-term EE has persistent effects on learning whereas short-term EE does not}

Once that I established the minimal amount of EE required to exert positive effects on learning and memory, my next aim was to find out whether that effect is persistent and if yes to what extent; i.e. will the mice who were enriched for 2 weeks still perform better on learning tasks after enrichment is terminated and they are housed in standard home cages? To investigate this, two different groups of mice were subjected to Morris water maze and Pavlovian fear conditioning paradigms; (i) mice that were kept in standard home cages (HC) and (ii) mice that were enriched for 2 weeks and were left in standard home cages for 1 week before being subjected to learning experiments ( $2 \mathrm{w} E \mathrm{EE}-1 \mathrm{w} \mathrm{HC}$ ). It turned out that the effects of 2 weeks of EE were only partly persistent; i.e. the mice in

A.

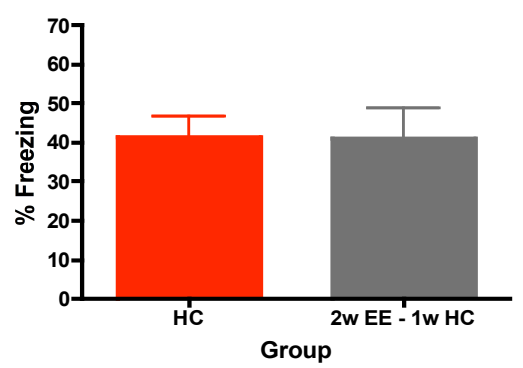

B.
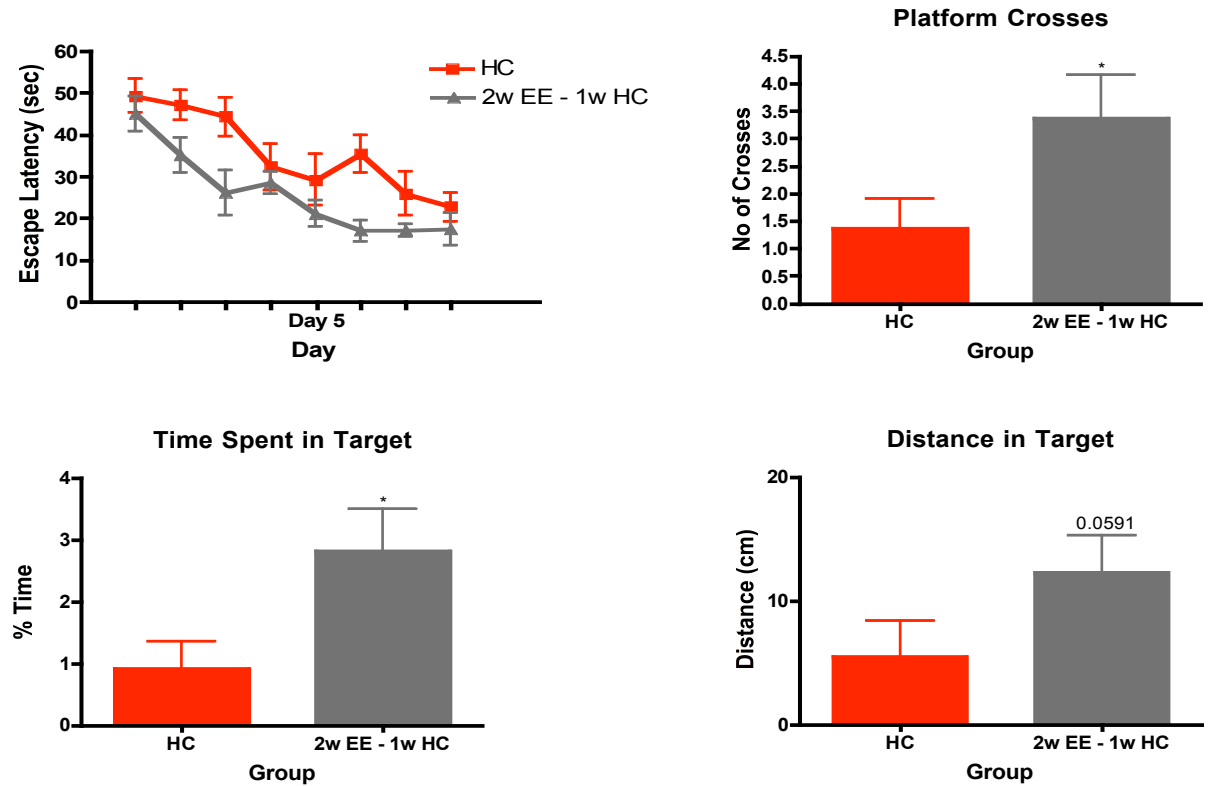

Figure 2.2: (A) 2w of EE do not have persistent effects on learning in Pavlovian fear conditioning $(\mathrm{n}=8)$.

(B) Mice that undergo $2 \mathrm{w}$ of EE continue to have facilitated memory even after being kept in home cage for 1 week (n $=8, p<0.05)$. 
the " $2 \mathrm{w}$ EE - 1w HC" group performed better than the home caged mice in the Morris water maze (Figure 2.2B), however unlike the mice which were enriched for 2 weeks and subjected to learning experiments right after that, the mice in the " $2 \mathrm{w} E E-1 \mathrm{w} \mathrm{HC}$ " group did not have increased memory in Pavlovian fear conditioning anymore (Figure 2.2A).

These findings made me wonder whether 2 weeks of EE would still have its positive effects on learning $\&$ memory in the water maze paradigm if the retention period would be extended far beyond 1 week. For this reason, the same mice that were used in previous experiment were still kept in home cages for up to 2 months, and then subjected to a novel water maze experiment. However, in this case it was necessary to ensure that the mice are really subjected to a completely novel task without any possible influence from the memory acquired in the previous water maze learning. Therefore, for this experiment the spatial cues were rearranged and the platform was put to another location. It was observed that when kept in home cage for 2 months after 2 weeks of EE the mice did not have enhanced memory anymore (Figure 2.3). Along with the result obtained from
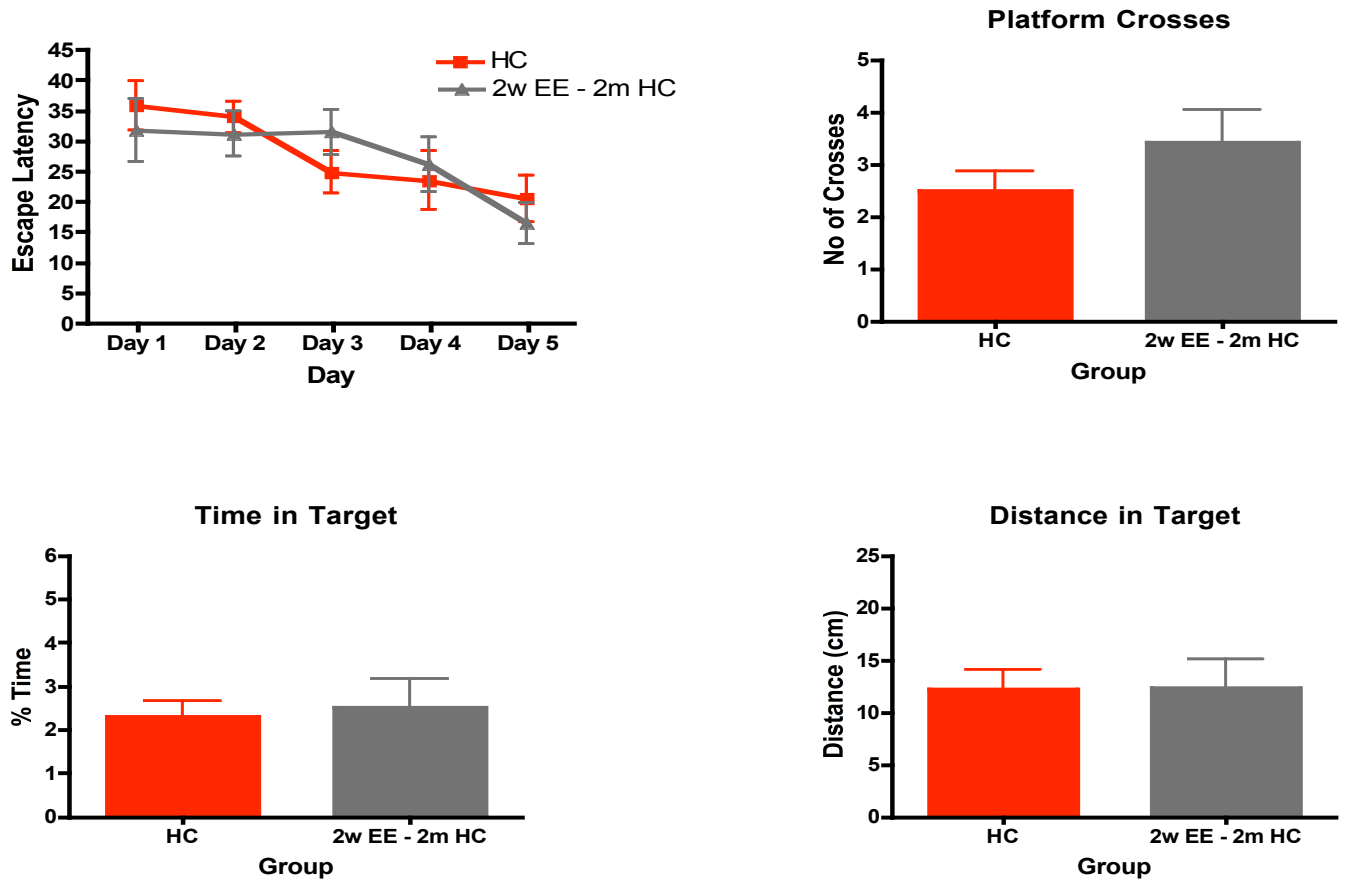

Figure 2.3: The positive effects of 2 weeks of EE on learning fade away when the retention period becomes much longer - i.e. 2 months $(n=8)$. 
Pavlovian fear conditioning this finding once again indicates that 2 weeks of environmental enrichment, although being enough to facilitate learning, do not have long-lasting effects on cognition.

A.

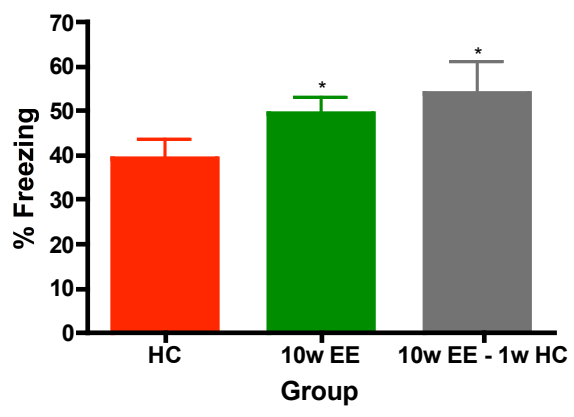

B.
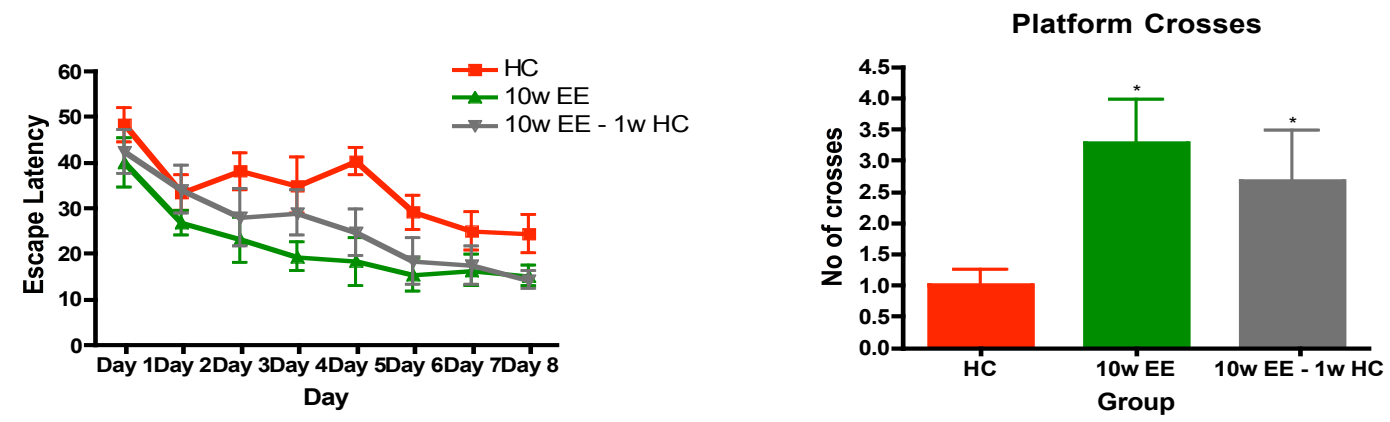

Time Spent in Target
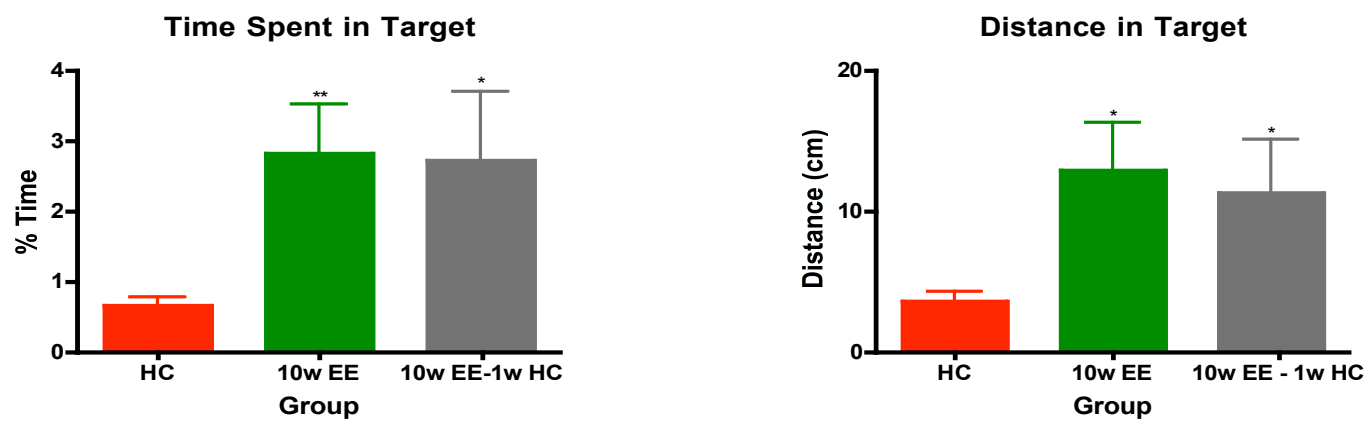

Figure 2.4: (A) 10 weeks of EE retain their positive effects on learning in fear conditioning task even after 1 week of retention $(\mathrm{n}=8, p<0.05)$.

(B) 10 weeks of EE have persistent effects on learning in water maze test as well $(\mathrm{n}=8, p<0.05)$. 
Next, I wondered whether a longer period of enrichment could exert fully long-lasting effects on cognition. For this purpose a group of mice was enriched for 10 weeks and then kept at home cage 1 week before being subjected to learning experiments. Another group was housed in enriched environment for 10 weeks and subjected to learning tests straight after that. A control group that was housed in standard home cages throughout the experiments was included as well. The first interesting observation was that the mice that underwent 10 weeks of $\mathrm{EE}$ and were kept in home cage for 1 week afterwards performed significantly better in the fear conditioning (Figure 2.4A) and water maze tasks (Figure 2.4B) compared to the home cage controls.

These observations already provided the first hint that in contrast to 2 weeks of EE, 10 weeks of EE should have long-lasting effects on cognition. Therefore, the same mice that were used for the experiments mentioned above were kept in home cages for up to two months. For this particular experiment the "10w EE" and "10w EE - 1w HC" groups were grouped together into one group, which was named " $10 \mathrm{w} \mathrm{EE}-2 \mathrm{~m} \mathrm{HC}$ ". The mice were then subjected to the modified water maze experiment mentioned above (see Figure 2.3). Interestingly, it was observed that even after being in the home cage for 2 months, the mice that underwent EE for 10 weeks still displayed increased learning (Figure 2.5). This indicates that long-term environmental enrichment exerts lasting effects on learning performance.
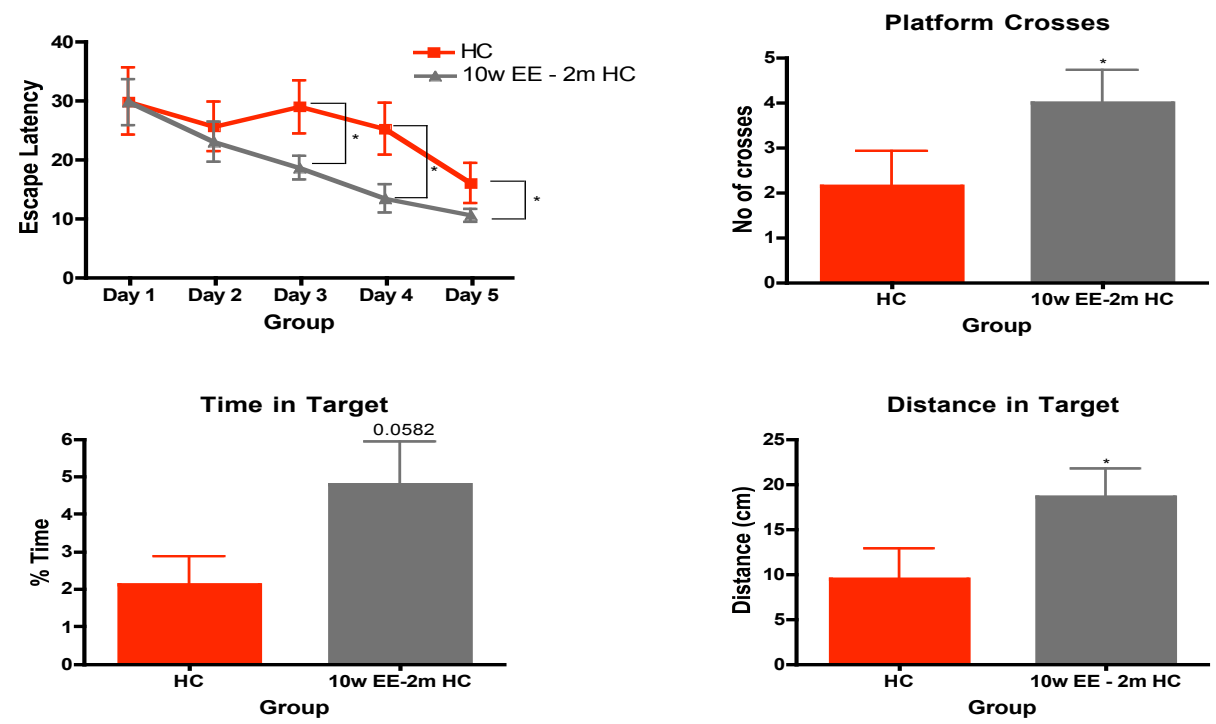

Figure 2.5: The positive effects of 10 weeks of EE on learning persist at least up to 2 months (HC: $\mathrm{n}=8 ; 10 \mathrm{w}-2 \mathrm{~m}$ $\mathrm{HC}: \mathrm{n}=16 ; p<0.05)$. 


\section{Long-term EE induces a substantial change in gene expression in hippocampus:}

The next question was whether long-term and short-term enrichment elicit their effects through different mechanisms, such that the effects of the former are long lasting and the effects of the latter are not. In order to dwell into this question gene expression in hippocampus of mice enriched for 10 weeks was compared to those housed in standard home cages, by DNA microarray. It was observed that 10 weeks of EE induced an extensive change in gene expression pattern in hippocampal tissue (Figure 2.6). There were 62 genes that were upregulated and 69 genes that were downregulated as a result of 10 weeks of EE (FDR: 0.05, fold change: 1.5). 16 genes were chosen for qPCR confirmation (Figure 2.7); for the qPCR experiment also another group of mice, the ones that underwent 10 weeks of $\mathrm{EE}$ and then were kept in home cage for 2 months, was included. Surprisingly, it was observed that among those 16 genes tested only one of them $(\mathrm{Ccl})$ retained its expression level induced by enrichment for up to 2 months after the cessation of EE (Figure 2.7).

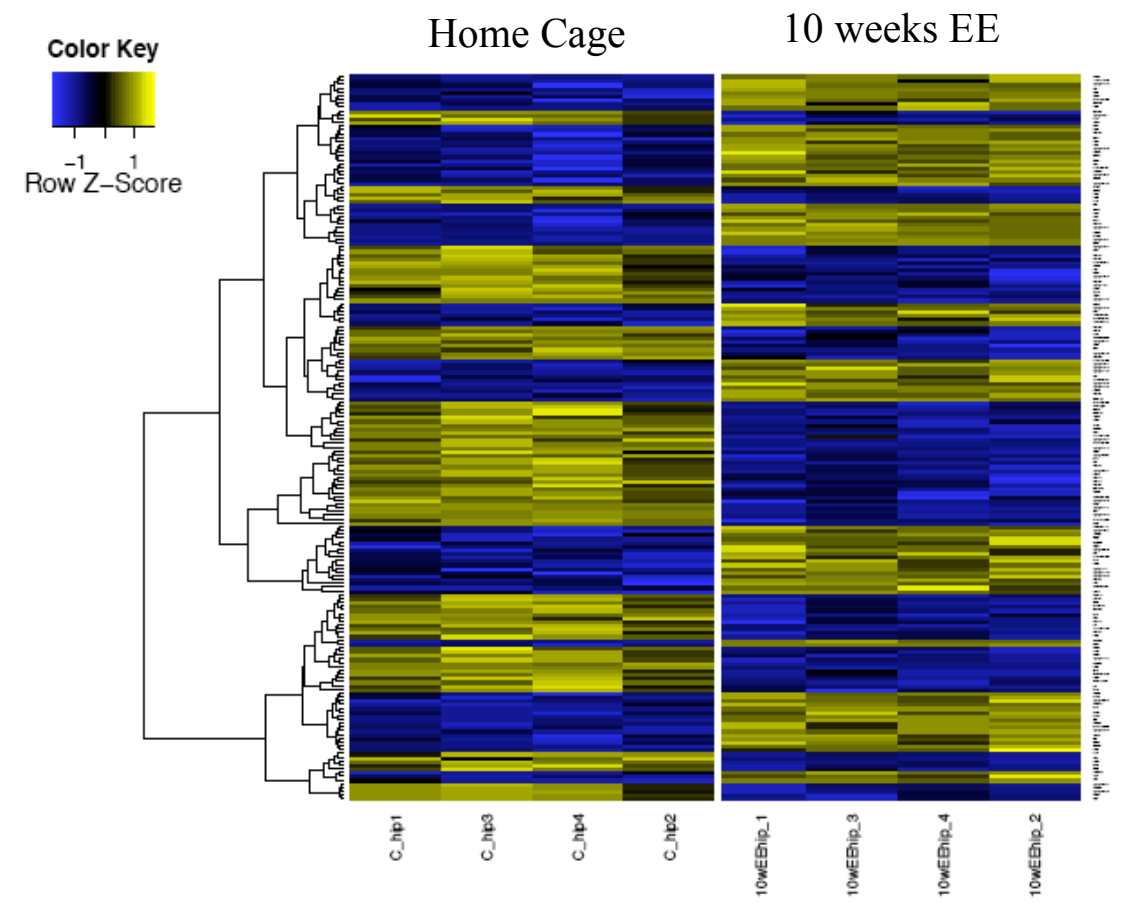

Figure 2.6: 10 weeks of EE induce upregulation in 62 and downregulation in 69 genes $(\mathrm{FDR}=0.05$; fold change $=1.5$; $\mathrm{n}=4)$. 


\section{Short-term enrichment induces only a subtle change in gene expression:}

The genes that were confirmed to be differentially regulated in response to 10 weeks of EE were also tested in the hippocampus of mice that underwent only 2 weeks of EE. It was observed that the effect on 2 weeks of $\mathrm{EE}$ on gene expression was very mild. Only 3 out of those 16 genes had a different expression level in hippocampus after 2 weeks of EE when compared to the control levels - Plbd1, Pde6h and Klf1 (Figure 2.8). 

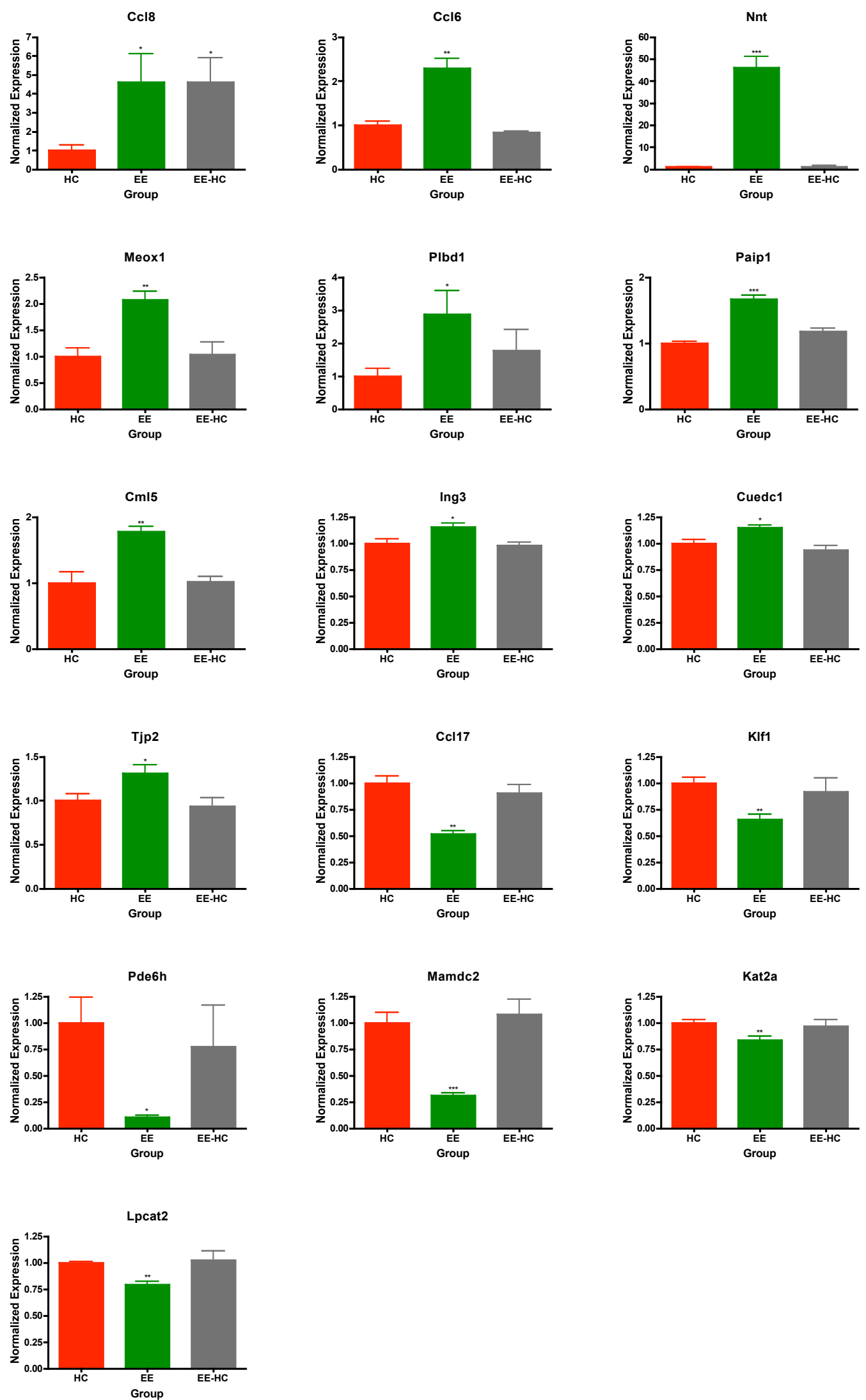

Figure 2.7: 16 of the genes that were differentially regulated in hippocampus after 10 weeks of enrichment ("EE" group) were chosen for qPCR confirmation. All of them except one $(\mathrm{Ccl} 8)$ returned to basal levels after the mice were transferred to home cages ("EE - HC" group) $\left(\mathrm{n}=4,{ }^{*} p<0.05, * * p<0.01\right)$. 

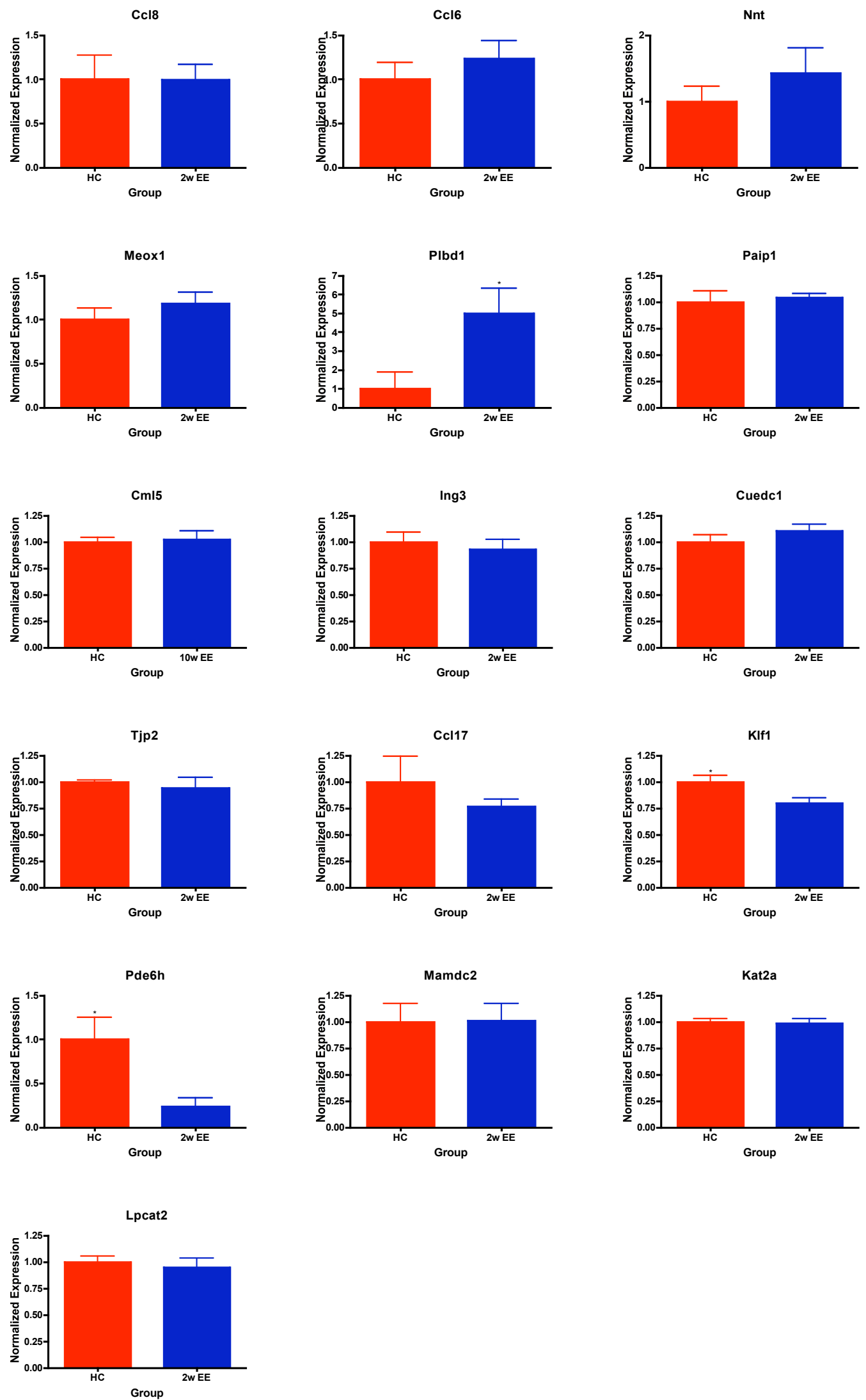

Figure 2.8: 15 of the genes that were differentially regulated in hippocampus after 10 weeks of enrichment ("EE" group) were chosen for qPCR confirmation. All of them except one $(\mathrm{Ccl} / 8)$ returned to basal levels after the mice were transferred to home cages ("EE - HC" group) ( $\mathrm{n}=8, * p<0.05)$. 


\section{DISCUSSION}

My thesis work consisted of two major directions. In one of them I analyzed the effects of knock-down of a histone methyltransferases (M112, in this case) in the forebrain on behavioural performance in mice and the molecular mechanisms underlying this. With this project I aimed to shed more light on the role of histone methylation in behaviour and specifically on learning \& memory.

In my other project I investigated the role of environmental enrichment in improving learning \& memory, with an emphasis on investigating to what extent its effects remain persistent after mice are removed from enriched environment.

Both of the projects yielded interesting results and venues for further research in the quest for deciphering the molecular mechanisms underlying learning \& memory.

\section{PART 1: CHARACATERIZATION OF FOREBRAIN SPECIFIC MLL2 CONDITIONAL KNOCK-OUT MICE}

\section{MIl2 knock-down in the forebrain and general characterization}

Knock-down of Ml12 was achieved through Cre - loxP recombination of the $2^{\text {nd }}$ exon of the gene. The exon2 of Mll2 gene was flanked by loxP sites and in addition an FRT sequence was inserted into the first intronic region $(F / F)$. Recombination of the "floxed" $2^{\text {nd }}$ exon through the action of Cre protein lead to a frameshift mutation which in turn gave rise to a premature stop codon on the $3^{\text {rd }}$ exon and hence prevented the translation of the mRNA into a functional protein. The occurrence of the frameshift mutation was in turn rendered possible by the presence of the above-mentioned FRT sequence. However, my aim was to induce the knock-down of Mll2 in the adult stage and specifically in the forebrain, and then to study the consequences of this manipulation on behaviour and molecular mechanisms underlying it. There are several reasons for this strategy rather than studying behavioural phenotype in mice with straight M112 knock-out. Firstly and most importantly, complete Ml12 knock-down was shown to be lethal after embryonic day 11.5 (E11.5) (Glaser et al., 2009). Secondly, having the gene of interest knocked down in the whole body would provide less information and even raise further questions 
with regards to its role in the observed behavioural phenotypes which are subserved only by certain brain parts (see 'Introduction'). Therefore the best way to study the effect of a gene, or of any manipulation for that matter, on cognition and behaviour is to restrict the knock-down of that gene to regions directly responsible for the cognitive and/or behavioural task under investigation. To achieve this aim conditional knock-out strategy has long been employed in various studies (Orban et al., 1992; Tsien et al., 1996; Zörner et al., 2003; Valor et al., 2011; McQuown et al., 2011).

In this case the conditional knock-down of M112 was achieved by crossing the mouse line having the exon 2 of Mll2 gene "floxed" to the mouse line expressing Cre recombinase under CamKII promoter. CamKII promoter in turn is only active in the forebrain glutamatergic (i.e. excitatory neurons) after postnatal day 19 (P19) (Mayford et al., 1996a; Mayford et al., 1996b; Tsien et al., 1996; Minichiello et al., 1999). As a result, loxP recombination of exon2 of Mll2 gene (i.e. Ml12 knock-down) is achieved only in forebrain excitatory neurons at the end of the third week after birth (F/F CKII). This strategy has previously been used successfully in our lab (Kuczera et al., 2010).

Before proceeding with the knock-out mice I first set out to determine the relative expression level of Mll2 mRNA in different brain regions; relative mRNA levels were analyzed in three main forebrain regions important for learning \& memory - dentate gyrus, CA and prefrontal cortex. The mRNA analysis showed that the expression of Mll2 is slightly lower in the CA region and prefrontal cortex compared to the dentate gyrus. This may indicate that M112 might play a more important function in the dentate gyrus compared to the other two brain regions. However, the difference in expression was not so high and did not reach significance to rule out the importance of M1l2 function for CA and prefrontal cortex as well at this stage. Next, I set to confirm the loxP recombination of Mll2 and also the knock-down of the protein. For the confirmation of recombination a qRT-PCR was performed with one of the primers targeting the $2^{\text {nd }}$ exon (the "floxed" one) and the other one targeting the $1^{\text {st }}$. The qRT-PCR results showed a 4 to 5 fold downregulation of the wild type transcript of Mll2 in dentate gyrus, CA and prefrontal cortex but not in cerebellum in F/F CKII mice.

M112 protein has a very big size $(400 \mathrm{kDa})$ and hence it has been difficult to find an antibody which would recognize it in brain samples. Therefore, the staining for M112 
protein - both in wild-type samples comparing its level in different brain regions and also in knock-out samples to confirm the knock-down of the protein - in different brain regions has not been performed up to now. However, as of now it is in progress and the staining protocol is being optimized by Dr. Andrea Kranz from Technical University of Dresden. Indeed, the specific antibody against M112, which is currently being optimized for immunoblot in hippocampal, prefrontal cortical and cerebellar samples from $\mathrm{F} / \mathrm{F}$ and F/F CKII mice, has been used successfully before by the same group (Glaser et al., 2006; Glaser et al., 2009). However in those studies the staining was done in embryonic stem (ES) cells, testis and whole brain lysates. The reason why Mll2 staining with the same antibody was observed in testis and whole brain lysates but not in separate brain regions could simply be that the former two are of much bigger size compared to the latter and hence simply contain more proteins in the first place. And the reason for observing M112 staining in ES cells could be that endogenous Mll2 expression is higher in ES cells than in separate brain regions.

One thing that needs to be mentioned is that there was no complete knock-down of Mll2 in neither of the forebrain regions examined. One reason for it is that the knock-down of M112 was achieved only in excitatory neurons, which means the inhibitory neurons and glial cells were spared. Another reason could be that this knock-out technology does not achieve the knock-down of the gene of interest in all the excitatory neurons (Valor et al., 2011). Nevertheless, the extent of downregulation was still large enough in order to observe a behavioural and molecular phenotype.

Next, it was necessary to see whether the knock-down of Mll2 induces some compensatory changes in expression of its close homologues Mll1 and Mll3. However, there was no such change in neither of the regions examined (dentate gyrus, CA and prefrontal cortex). These results strongly suggest that the behavioural and/or molecular phenotype that will be observed in these mice will not be due to interference from the function of Mll1 and/or Mll3.

The brain size and weight of the F/F CKII mice were comparable to those of controls. This is expected in view of the fact that in F/F CKII mice knock-out of Mll2 occurs not earlier than the $3^{\text {rd }}$ week after birth. Although in rodents development of certain brain parts continues for a certain amount of time after birth (Akazawa et al., 1995; Clinton et 
al., 2000; Zhang et al., 2005), nothing of a potentially gross effect on whole brain morphology occurs from the $3^{\text {rd }}$ week after birth. Consistent with this observation there were also no gross abnormalities in hippocampal morphology observed by NeuN staining. There was however a slight decrease in MAP2 staining in stratum radiatum and synaptophysin staining in stratum lucidum of F/F CKII mice which nevertheless failed to reach significance. MAP2 (microtubule associated 2) is a cytoskeletal protein present in the dendrites, and playing a role in stabilizing dendritic shape during synapse development (Caceres et al., 1992; Johnson and Jope, 1992; Gamblin et.al., 1996; Lim et al., 2000). Synaptophysin in turn is an integral membrane protein of synaptic vesicles (Jahn et al., 1985; Wiedenmann and Franke, 1985; Südhof et al., 1987; McMahon et al., 1996). These observations could therefore indicate that although they do not have any gross defects in brain morphology, synapse development and/or fine-tuning of synaptic connections may be slightly impaired in F/F CKII mice.

The body weight of the male F/F CKII mice was comparable to that of their control counterparts. However, female knock-out mice showed a slightly but nevertheless significantly increased body weight.

\section{MII2 knock-down in the forebrain leads to increased anxiety and learning deficits}

Once the knock-down of M112 in the forebrain was confirmed the next step was to investigate the effect of this on behaviour. The mice were subjected to a behavioural battery that consisted of paradigms testing for anxiety and learning \& memory. The Mll2 F/F CKII mice were observed to spend significantly less time in the centre of an open field arena compared to F/F mice. However, the total distance travelled by those two groups during a 5 min exploration was similar. Open field test is a widely used paradigm to test anxiety. The rationale behind the test is that the centre of the open field appears less safe compared to the periphery; and therefore a relatively anxious mouse will tend to spend more time in the centre compared to a less anxious one. In other words, the readout for anxiety in this test is the relative amount of time spent in the centre of the open field arena - the more anxious the mouse the less time it will spend in the centre. In view of this it becomes apparent that the knock-down of Mll2 in the forebrain leads to elevated 
anxiety in mice without affecting general explorative behaviour. The same phenotype was observed in male and female mice separately and there was no sex specific effect or sex-genotype interaction. In order to confirm this finding, the mice underwent another test for anxiety namely elevated plus maze test. The rationale of this test is similar to the one employed in the previous one - utilizing the aversion of mice from open and less protected spaces. The elevated plus maze test is performed on a plus maze with four arms which are elevated above the ground. Two of the arms are surrounded by walls and two of the arms are open. And the readout for anxiety in this case is the relative amount of time spent in the open arms. Since the open arms are less safe a relatively anxious mouse will tend to spend less time in the open arms of an elevated plus maze compared to a less anxious one. And in accordance with the previous finding from the open field test the F/F CKII mice spend relatively less time in the open arms of the elevated plus maze compared to their control counterparts during a $5 \mathrm{~min}$ exploration. And in this case as well, males and females showed the same trend when examined separately and there was no sex specific effect or sex-genotype interaction.

Next, I set out to study the effect of selective Mll2 knock-down in the forebrain on learning \& memory. The first test that I used for this purpose was novel object recognition (NOR). This paradigm utilizes the natural proclivity of rodents to explore objects that are not familiar to them - i.e. the objects that they have not encountered before. During the training phase mice are put into an open field arena and are introduced to two similar objects (i.e. of the same size, shape and colour). They are left to explore them for a certain amount of time (it was 5 min in this study) and then are put back into the home cages, retained there and then are put back into the open field with the objects for the actual memory test. But this time one of the objects is exchanged to a new one; and the readout for memory is the relative preference that the mouse shows to exploring the new object over the old one (i.e. the one encountered during the training phase) (see 'Materials and Methods' for details). The amount of time that the mouse is retained in the home cage between the training phase and the memory test depends on the type of memory being investigated (see 'Introduction'). It has been observed that when the mice are introduced to the novel object after $5 \mathrm{~min}$ of retention the F/F CKII mice fail to show a preference to the novel object over the old one. This clearly indicates that knock-down 
of M112 leads to impairment in memory which does not depend on gene transcription but rather utilizes already existing synapses an signalling machinery (e.g. pre and postsynaptic proteins, signalling molecules etc.). This is supported by my previous observation that F/F CKII mice show a decrease in MAP2 and Syp staining in stratum radiatum and stratum lucidum respectively compared to their control counterparts. 24 hours later the mice were put back into the same open field arena and this time presented with the same old object and with another novel object different from the previous one. And in this case as well, the mice having their Mll2 gene knocked-down in the forebrain glutamatergic neurons do not show preference to the novel object over the old one. This indicates that M112 knock-down leads to impairment in long-term memory which is dependent on novel protein synthesis and gene expression. As a result it is tempting to speculate that Mll2 plays an important role in activity-induced transcription as well. However, a simpler and more straightforward explanation can also be provided to this phenomenon. Since there already seems to be an impairment in pre and/or post-synaptic signalling mechanisms and since synaptic signalling constitutes the initial stage of the whole signalling machinery which eventually converges onto the nucleus and mediates gene expression in response to a learning stimulus, it should come as no surprise that having the upstream portion of the machinery already impaired would lead to the impairment of the machinery as a whole. In this case the role of Mll2 in gene expression dependent memory would be only secondary. However, this explanation still does not exclude the possibility of M112 also having a role in additional gene expression induced by learning-related stimuli. A possible future strategy to dissociate the role of M112 in constitutive expression of genes involved in synaptic and neuronal function from its role in mediating learning-related stimulus dependent transcription would be to subject $\mathrm{F} / \mathrm{F}$ mice to training and short-term memory test but shortly after that to induce M112 knockdown through Cre-recombination by injecting adenovirus bearing CamKII-Cre construct, and then 24 hours later test the mice for long-term memory. However one caveat with this approach would be that it is quite difficult to find an adenovirus which would be expressed and have functional consequences 24 hours after injection.

The next learning test the mice were subjected to was Pavlovian fear conditioning. This paradigm tests for associative memory. Mice are put into a chamber with the floor 
consisting of metal grids, and are allowed to explore it for 3 min. After that they receive a mild foot-shock (see 'Materials and Methods' for details). And when they are put back into the same chamber 24 hours later without receiving any electric foot-shock this time, they associate the context with the unpleasant experience that they had in this same context 24 hours ago (i.e. the foot-shock) and as a result they show freezing which is the sign of fear. Freezing behaviour is an inborn behaviour which rodents manifest when they are confronted with a potentially dangerous situation. Therefore, in the Pavlovian fear conditioning paradigm the readout for memory strength is the level of freezing that rodents show when they are subjected to the same context. Simply put, the stronger the memory the more the freezing. In view of this fact it becomes obvious that F/F CKII mice have a slight impairment in associative memory, since they show significantly lower freezing when introduced into the same context 24 hours later after receiving the a $0.5 \mathrm{~mA}$ foot-shock. Again the same phenotype is observed in males and females separately. One interesting observation is that when they were subjected to a stronger foot-shock $(0.7 \mathrm{~mA})$ the memory impairment was absent in F/F CKII mice. This indicates that M112 knock-down in the forebrain leads to an impairment in associative memory only when the task becomes more challenging. Another explanation for the mild phenotype that was observed in F/F CKII mice in fear conditioning may come from the observation that they also manifest anxiety (see above). The freezing level in the fear conditioning paradigm is not dependent only on the strength of associative memory but also on the level of anxiety (Sadler et al., 2011; Sartori et al., 2011); with increased anxiety there will be an increase in the freezing level. Therefore it is quite possible that the results from the fear conditioning experiment underestimate the level of memory impairment in F/F CKII mice. There are two interesting experiments that could be performed in the future. One is to test the short-term memory in Mll2 F/F CKII mice in contextual fear conditioning - i.e. to test their freezing level when they are reintroduced into the same context 1 hour later. Another one is to test their associative memory in cued fear conditioning paradigm. In this test rodents are again allowed to explore the fear conditioning chamber for 3 minutes under the accompaniment of a sound, and after that they receive a foot-shock. During the memory test they are put into a different context but provided with the same sound that was present during the training phase, and the level of 
freezing that they manifest shows their associative memory towards the cue (i.e. the sound). Cued fear conditioning and contextual fear conditioning are subserved by different brain regions - the former by amygdala and the latter by hippocampus.

The last memory test to which the mice were subjected was Morris water maze. It tests the ability of rodents to find a platform hidden beneath opaque water using the spatial cues in the room. It tests for spatial memory which is highly dependent on hippocampus. At the end of the training, which in this study lasted for 2 weeks, the mice are subjected to the probe test in which their persistence to swim in the area where the platform was previously located indicates the strength of the memory (see 'Materials and Methods' for details). During the training phase of 2 weeks F/F CKII mice already showed a higher escape latency to reach the platform compared to the F/F mice, which especially became obvious during the last days of training. And during the probe test F/F CKII mice failed to show a preference for the quadrant where the platform was previously located (i.e. target quadrant designated as "T"). This indicates that apart from inducing anxiety, impairing object memory and associative memory, Mll2 knock-down also impairs spatial memory.

Stress and anxiety are mediated by an intricate circuitry utilizing hippocampus (ventral hippocampus in particular as will be discussed below), amygdala and medial prefrontal cortex (mPFC) (Campeau and Davis, 1995; Müller et al., 1997; Oddie and Bland, 1998; Coutureau et al., 2000; Gray and McNaughton, 2000; LeDoux, 2000; Petrovich et al., 2001; Bannermann et al., 2004; Degroot and Treit, 2004; Bertoglio et al., 2006; Pentkowski et al., 2006; Nascimento Häckl and Carobrez, 2007; Etkin et al., 2011). All of those three structures are connected to each other through bilateral connections (Maren and Fanselow, 1995; Gray and McNaughton, 2000; Vertes, 2006; LeDoux, 2000; Price, 2007; Canteras et al., 2010), and each of them in turn projects to hypothalamus the main structure of the brain involved in neuroendocrine functions mediating defensive behaviour, which comprises anxiety behaviour as well (Risold and Swanson, 1996; Canteras et al., 1997; Risold et al., 1997; LeDoux, 2000; Dielenberg et al., 2001; Canteras, 2002; Blanchard et al., 2003; Markham et al., 2004; Blanchard et al., 2005; Gabbott et al., 2005). Several studies have shown that lesion and/or inactivation of 
ventral hippocampus or amygdala leads to a decrease in anxiety (Henke, 1990; Campeau and Davis, 1995; Müller et al., 1997; LeDoux, 2000; Kjelstrup et al., 2002; Gale et al., 2004; Pentkowski et al., 2006; Nascimento Häckl and Carobrez, 2007). In other words, this indicates that hippocampal and amygdalar activity facilitates anxiety behaviour and therefore inhibition of these structures reduces anxiety. Apart from lesion studies also studies on knock-out mice which have impairment in hippocampal function showed repeatedly that they have decreased anxiety (Abeliovich et al., 1993; Izquierdo and Medina, 1995; Masugi et al., 1999; Contarino et al., 1999; Wall and Messier, 2000; Bowers et al., 2000; Otto et al., 2001a; Otto et al., 2001b; Bontekoe et al., 2002; D’Adamo et al., 2004; Greco and Carli, 2006). One thing that needs to be borne in mind is that decreased hippocampal dysfunction also leads to decreased learning and memory, and therefore it has until lately been perceived as a conceived wisdom that learning impairment should correlate with decreased anxiety (Izquierdo and Medina, 1995) which would contradict my findings where Mll2 F/F CKII mice have increased anxiety together with decreased learning. However, things did not turn out so simple; prior to my findings contradicting this conceived wisdom several other studies showed that transgenic mice having impairments in learning and memory had increased anxiety (Müller et al., 1994; Parks et al., 1998; Ross et al., 2000; Sarnyai et al., 2000; Sibille et al., 2000; Dirks et al., 2001; Guadano-Ferraz et al., 2003; Bannerman et al., 2004; Venero et al., 2005). In other words, memory and anxiety phenotypes do not always follow a linear pattern but rather co-occur in a more complex manner (Ribeiro et al., 1999; El Hage et al., 2004; Bierman et al., 2005; El Hage et al., 2006; Kalueff, 2007). Moreover, things are exactly the opposite with regards to the involvement of medial prefrontal cortex (mPFC) in anxiety behaviour. Inhibition of mPFC function was shown to increase anxiety and exposure to anxiogenic stimuli (e.g. elevated plus maze, open field) was shown to elicit and increase in Fos expression in that region (Sewards and Sewards, 2002; Bishop, 2007; Canteras et al., 2010). Therefore the reason for increased anxiety in Mll2 F/F CKII mice could lie in dysfunction of mPFC. For this reason a further venue of research on the way of discerning the molecular mechanisms underlying the increased anxiety in Mll2 F/F CKII mice would be analysing changes in gene expression in mPFC by DNA microarray or by 
RNA-Seq. Of special interest would be genes that would be downregulated specifically in $\mathrm{mPFC}$ of F/F CKII mice but not in dentate gyrus.

\section{MII2 activates transcription of genes important for neuronal function in dentate gyrus}

Although ventral part of hippocampus is involved in anxiety behaviour, the dorsal part of hippocampus is involved in learning \& memory (esp. spatial memory) (Henke, 1990; Jung et al., 1994; Moser et al., 1995; Moser and Moser, 1998; Pothuizen et al., 2004). The afferent and efferent connections that these two parts of hippocampus make with other brain regions are distinct from each other (Swanson and Cowan, 1977; Fanselow and Dong, 2010). Moreover, apart from differences in anatomical connections hippocampus also manifests differences in gene expression along dorso-ventral axis (Thompson et al., 2008; Dong et al., 2009; Fanselow and Dong, 2010). Since Ml12 knock-down leads at the same time to learning and anxiety phenotypes it was important to dissociate dorsal and ventral parts of hippocampus when proceeding with molecular analysis. Surprisingly, dissection of dorsal and ventral parts of mouse hippocampus has never been performed by hand; the data that exist concerning the differences in gene expression along dorso-ventral axis come from in situ hybridization (Thompson et al., 2008; Dong et al., 2009; Fanselow and Dong, 2010). That is why before proceeding further it was important for me to test whether we can faithfully isolate dorsal and ventral parts of hippocampus by dissection by hand. For this purpose I selected some genes which have been shown before to show dorso-ventral specificity in hippocampus (Thompson et al., 2008; Dong et al., 2009; Fanselow and Dong, 2010) and compared their expression levels in dorsal versus ventral hippocampal samples by qRT-PCR. I managed to confirm the dorsal and ventral specific genes in the tissue samples, which indicated that our way of dissecting dorsal and ventral hippocampus is reliable.

Then, I decided to concentrate on learning impairment caused by M112 knock-down and therefore proceeded with dorsal part of dentate gyrus and CA. DNA microarray was performed from dorsal dentate gyrus and dorsal $\mathrm{CA}$ of $\mathrm{F} / \mathrm{F}$ and $\mathrm{F} / \mathrm{F}$ CKII mice in order to see whether knock-down of M112 lead to changes in gene expression in those regions. 
Quite intriguingly, it was observed that M112 knock-down lead to an extensive donwregulation of gene expression in dorsal dentate gyrus which is expected in view of the fact that Mll2 mediates Histone 3 Lysine 4 methylation which is a mark activating gene expression; however there was no such drastic downregulation in dorsal CA region. This can partly be explained by slightly higher expression of Mll2 in the dentate gyrus, however still cannot account for such a drastic difference in the number of donwregulated genes. This finding indicates that M112 might play a special role confined to dentate gyrus, whereas not being that important for regulation of gene expression in CA region. There were also some upregulated genes in dorsal dentate gyrus of F/F CKII mice which can be accounted for by the secondary effects resulting from Ml12 knock-down.

Among downregulated genes there were many which are involved in molecular processes and/or pathways known to be involved in synaptic signalling and synaptic plasticity. For example, there were many genes (Nkapl, Adcy5, Dusp2, Prkra) that code for proteins that are involved in G-protein - cAMP and MAPK/ERK pathways, which have many times been shown to be crucial for synaptic plasticity in hippocampus and learning \& memory (Frey et al., 1993; English and Sweatt, 1996; English and Sweatt, 1997; Atkins et al., 1998; Impey et al., 1998; Roberson et al., 1999; Sweatt, 2001; Levenson et al., 2004; Chwang et al., 2006; Wood et al., 2006; Reissner et al., 2006; Sindreu et al., 2007; Brami-Cherrier et al., 2007; Ehninger et al., 2008; Brami-Cherrier et al., 2009; Reul et al., 2009). Two genes that deserve special attention here is NF- $\mathrm{kB}$ activating protein like (Nkapl) and adenylate cyclase 5 (Adcy5). NF- $\mathrm{\kappa B}$ signalling has repeatedly been shown to be crucial for synaptic plasticity and learning \& memory (Meberg et al., 1996; Albensi and Mattson, 2000; Yeh et al., 2002; Freudenthal et al., 2004; Lubin and Sweatt, 2007; Ahn et al., 2008), and adenylate cyclases are the enzymes involved in the synthesis of cAMP whose role in synaptic plasticity and learning \& memory is also well established (see above and 'Introduction'). An interesting observation here is that dual specificity phosphatase 2 (Dusp2) is among the genes that are downregulated in F/F CKII mice. Dual specificity phosphatases dephosphorylate MAP kinases and function to inactivate MAP/ERK signalling pathway (Charbonneau and Tonks, 1992). Indeed, it has been shown that another dual specificity phosphatase - MCF-1, also known as Dusp1 - is rapidly transcribed both in fibroblasts after stimulation with serum growth factor (Lau 
and Nathans, 1985; Sun et al., 1993; Charles et al., 1993), and also in hippocampus or specifically in granule cells of dentate gyrus after induction of LTP (Qian et al., 1993; Qian et al., 1994; Davis et al., 2000). At first it may come as a surprise that firstly something that actually inactivates a pathway involved in synaptic plasticity is induced after neuronal stimulation, and secondly that something else of a similar type is downregulated in the dentate gyrus of mice that have learning impairment. However, the fact that a dual specificity phosphatase is rapidly transcribed upon induction in order to inactivate $\mathrm{MAP} / \mathrm{ERK}$ signalling may indicate that it is important in regulating the feedback loop which in turn renders the pathway responsive to the next stimulus. Indeed, it has been suggested that activity of phosphatases confers flexibility to the MAPK/ERK signalling pathway making it respond to a stimulus acutely (Bhalla and Iyengar, 1999; Bhalla et al., 2002). Seen in this light it makes perfect sense that activity of MAPK phosphatases are important for the proper functioning of MAPK/ERK pathway and for occurrence of phenomena induced by it (e.g. long-term potentiation). It is highly likely that dephosphorylating the MAPK is necessary to make it ready to respond to the next stimulus and hence a failure in the molecular mechanisms responsible for MAPK dephosphorylation may lead to its unresponsiveness and lack of flexibility and may result in deficits in synaptic plasticity. Therefore induction of expression of gene(s) coding for MAPK dephosphotase(s) after LTP induction might serve to revert MAPK to the original state ready to respond to later stimuli. However, this might be achieved not only through online induction but also through constitutive expression of MAPK dephosphatases, a decrease in expression of which might as well lead to deficits in synaptic plasticity and learning \& memory. And this is what we observe in the case of M112 F/F CKII mice; downregulation of Dusp2 expression in dentate gyrus is highly likely to leave certain MAP kinase(s) hyperphosphorylated already at the basal level hence rendering them incapable of responding to upcoming stimuli.

Another set of genes that were downregulated in dorsal dentate gyrus as a result of Mll2 knock-down code for proteins involved in synaptic vesicle transport and fusion (e.g. Stxbp2, Sypl2, Apls3 and Ckap4). Others code for transcription factors involved in transcriptional initiation (e.g. E2fl) and transcriptional elongation (e.g. Tceall). Different transcriptional activators (e.g. CREB, Fos, Egr1, Egr2, Jun, Arc) have previously been 
shown to be involved in synaptic plasticity and LTP induction (Impey et al., 1996; Deisseroth et al., 1996; Impey et al., 1998; Taubenfeld et al., 1999; Tischmeyer and Grimm, 1999; Albensi and Mattson, 2000; Lee et al., 2004; Frankland et al., 2004; Thomas and Huganir, 2004; Ramanan et al., 2005).

Another set of downregulated genes previously shown to facilitate synaptic plasticity and learning \& memory (Sun and Alkon, 2001) comprises carbonic anhydrases (Car4 and Car10). What is more interesting in the findings of Sun and Alkon is that presence of carbonic anhydrase activators facilitated spatial learning by inducing a switch in GABAergic responses from inhibitory to excitatory. Indeed it has previously been shown that GABAergic synaptic responses can be switched from inhibitory to excitatory (Alkon et al., 1992; Collin et al., 1995; Kaila et al., 1997; Sun et al., 2000; Sun et al., 2001). All these become quite intriguing given the fact that the gene coding for GABA receptor subunit $\gamma 3$ (Gabrg3) is also downregulated in dorsal dentate gyrus of F/F CKII mice. It is highly likely that decrease in expression of carbonic anhydrases and also the GABA receptor lead to the inability of F/F CKII mice to utilize GABAergic inputs for learning purposes - i.e. there most probably is a decrease in GABAergic signalling in the first place but also the remaining GABAergic signals are most probably not being converted to excitatory ones hence leading to deficits in learning \& memory. Moreover, neither Car4 nor Gabrg3 is downregulated in the ventral part of dentate gyrus in F/F CKII mice (see below), which makes perfect sense in light of findings indicating that GABAergic switch is important for spatial memory (see above). Therefore involvement of Mll2 in hippocampus dependent memory (esp. spatial memory) can at least partly be attributed to its regulating the expression of Car4 and Gabrg3 specifically in dorsal part of dentate gyrus.

There were also genes coding for proteins involved in lipid metabolism - Ptgr1, Ptgr2, Acot1 and Acot4. Another gene that deserves attention is Oxr1 which is involved in protecting cells against oxidative stress (Volkert et al., 2000; Elliott and Volkert, 2004; Durand et al., 2007). Moreover, in contrast to many others this gene was downregulated in dorsal CA as well. Increased oxidation has been shown to impair neuronal function and synaptic plasticity and in contrast reducing agents have been shown to facilitate LTP (Gozlan et al., 1995; Cai et al., 2008; Yang et al., 2010). Moreover, a recent study has 
implicated Oxr1 in protecting neuronal cells from oxidative stress and neurodegeneration (Oliver et al., 2011). That is why an interesting venue for future research would be to test whether Mll2 F/F CKII mice show increased neurodegeneration as they age compared to the controls. Moreover, since M1l2 apparently plays a more important role in dentate gyrus and appears to be dispensable for CA, it is tempting to speculate that M1l2 may also play a role in adult neurogenesis. One piece of evidence pointing to this possibility is that E2f1, which is downregulated in dorsal dentate gyrus of F/F CKII mice, has been shown to be involved in neurogenesis in adult mice (Cooper-Kuhn et al., 2002).

Another interesting observation was that among 16 genes confirmed to be downregulated in dorsal dentate gyrus by qRT-PCR most but not all were downregulated in the ventral part. The genes that were not downregulated in ventral dentate gyrus of F/F CKII mice are Adcy5, Gabrg3, Car4, Rab38 and Tpm4. This in turn points to the possibility that apart from its specificity for dentate gyrus, Mll2 also may show some specificity of function along dorso-ventral axis of hippocampus.

\section{Histone 3 Lysine 4 methylation is decreased at promoters and coding regions of genes downregulated in dentate gyrus as a result of MIl2 knock-down}

Since Ml12 methylates Histone 3 at Lysine 4 residue the next obvious step was to see whether there is a decrease in $\mathrm{H} 3 \mathrm{~K} 4$ methylation at the promoters of the genes downregulated in dorsal dentate gyrus in F/F CKII mice. And as expected I observed a decrease in $\mathrm{H} 3 \mathrm{~K} 4$ di and trimethylation, which are markers for active transcription, at the promoters of those genes. SET1 domain histone methyltransferases have repeatedly been shown to be responsible for $\mathrm{H} 3 \mathrm{~K} 4$ tri and dimethylation inside the cell (Miller et al., 2001; Santos-Rosa et al., 2002; Krogan et al., 2003; Ng et al., 2003; Bannister and Kouzarides, 2004; Hughes et al., 2004; Schneider et al., 2005; Tenney and Shilatifard, 2005; Dou et al., 2006; Sims and Reinberg, 2006; Bhaumik et al., 2007; Wood et al., 2007; Malik and Bhaumik, 2010; Ansari and Mandal, 2010). An interesting phenomenon observed previously is that promoters and coding regions of constitutively active genes are marked both by H3K4me3 and H3K4me2 (Santos-Rosa et al., 2002). In other words, it follows that both $\mathrm{H} 3 \mathrm{~K} 4$ tri and dimethylation marks are responsible for mediating 
transcription of genes at the "naïve" state. And my findings regarding the effect of knockdown of M112 on gene expression changes and H3K4 methylation in the dentate gyrus are perfectly consistent with this notion. The mice, which were used for DNA microarray and chromatin immunoprecipitation analyses were not subjected to any stimulus prior to being sacrificed. Therefore the genes downregulated in Mll2 F/F CKII mice are expected to have at least an appreciable level of transcription at the basal (i.e. "naïve") state. And since H3K4 tri and dimethylation are responsible for mediating the transcription of constitutively active genes (Santos-Rosa et al., 2002), it is absolutely expected that genes downregulated in "naïve" mice as a result of M1l2 knock-down will have reduced trimethyl and dimethyl marks at their promoters and/or coding regions. However, another interesting finding from the study of Santos-Rosa et al. was that only H3K4 trimethylation but not dimethylation was responsible for transcriptional activation of genes whose activity is regulated in response to a stimulus (Santos-Rosa et al., 2002). In light of this observation it would be interesting to compare the transcription profile of control and M112 F/F CKII mice in response to a learning stimulus. However, in this case the analysis would be more complicated since F/F CKII mice show downregulation in many genes, which are actually involved in mediating transcriptional activation in response to a stimulus - either directly like in the case of transcription initiation/elongation factors (e.g. E2fl and Tceall) or through being part of signalling cascades that lead to changes in gene expression (e.g. Nkapl, Adcy5, Dusp2, Prkra). That is why the differences in regulated gene expression that could possibly be observed between control and Mll2 F/F CKII mice may not be only due to impaired H3K4 trimethylation but also due to secondary effects resulting from already existing differences in gene regulation machinery. One possible way to disentagle this caveat could be to analyze $\mathrm{H} 3 \mathrm{~K} 4 \mathrm{me} 3$ level throughout the genome after a learning stimulus by ChIP-Seq analysis, and compare this in control and F/F CKII mice.

Another interesting finding was that in contrast to decrease in H3K4 trimethyl and dimethyl marks there was no change in monomethyl mark at gene promoters in F/F CKII mice, and for some genes (Car4, Adcy5, Stxbp2 and Acot4) it was even increased or tended to increase in the knock-outs. In fact it was shown by Andreu-Vieyra et al. that Ml12 knock-down is required for bulk H3K4 trimethylation and dimethylation, but not 
monomethylation in oocytes (Andreu-Vieyra et al., 2010). My findings from dorsal dentate gyrus are in line with the results obtained from that study. These all indicate that either in the mammals $\mathrm{H} 3 \mathrm{~K} 4$ monomethylation is carried out by some other histone methyltransferase(s) and that M112 is not responsible for that at all, or that a possible monomethylase activity of M112, but not dimethylase and trimethylase activity, can be compensated by other enzymes inside the cell.

\section{Injection of Histone Deacetylase (HDAC) inhibitor does not increase expression of genes downregulated as a result of MII2 knock-down}

It has previously been shown that $\mathrm{H} 3 \mathrm{~K} 4$ methylation serves as a mark that facilitates the recruitment of Histone Acetyltransferases (HATs) at gene promoters (Wang et al., 2009). Therefore it is likely that impairment of $\mathrm{H} 3 \mathrm{~K} 4$ methylation may lead to impairment in mediating histone acetylation as well. For this purpose I decided to compare the response of $\mathrm{F} / \mathrm{F}$ and $\mathrm{F} / \mathrm{F}$ CKII mice to injection of Suberoylanilide hydroxamic acid (SAHA), a potent inhibitor of histone deacetylases. It has been shown previously in our lab that intraperitoneal SAHA injection leads to an increase in bulk histone acetylation levels in hippocampus, with the peak of this increase being at 1 hour after injection (our unpublished observations). Firstly, I confirmed this observation in the Mll2-Cre mouse line, and observed that bulk histone acetylation increases in hippocampus 1 hour after SAHA injection both in F/F and also in F/F CKII mice. This indicates that F/F CKII mice at least do not have impairment in regulating bulk histone acetylation, and that 1 hour after injection is the best time point to further study the effects of SAHA in more detail. Still, the fact that Ml12 knock-down does not lead to impairment in increasing bulk levels of histone acetylation after SAHA treatment does not mean that there will not be any impairment in regulation of expression of genes already shown to be downregulated in dentate gyrus of F/F CKII mice. Therefore, I set out to investigate whether SAHA injection leads to an increase in expression of those genes in dorsal dentate gyrus in $\mathrm{F} / \mathrm{F}$ and F/F CKII mice. Interestingly, it turned out that those genes are not upregulated in dorsal dentate gyrus 1 hour after SAHA injection neither in F/F CKII nor in F/F mice. This was quite an unexpected finding; the expectation was that those genes will be 
upregulated at least in the control mice after SAHA injection but not in F/F CKII mice. At this stage this finding may indicate two things, not necessarily mutually exclusive. Firstly, it is possible that in the brain genes regulated by histone methylation are not regulated by an additional increase in histone acetylation. However, one more crucial experiment is necessary in order to definitely prove this hypothesis; it should be tested by chromatin immunoprecipitation (ChIP) whether acute SAHA injection increases histone acetylation at the promoters of those genes in the first place. Another interesting possibility, which would be more intriguing, is that by itself acute SAHA injection and the resulting increase in histone acetylation do not lead to any changes in gene expression in the brain at all, but rather only create a permissive state at the chromatin so that genes are transcribed faster and/or at a higher level only when a necessary stimulus arrives. Interestingly, all the previous studies demonstrating the effect of acute injection of HDAC inhibitors on gene expression in hippocampus always coupled it to another stimulus - e.g. fear conditioning (Vecsey et al., 2007; Peleg et al., 2010). The only obvious way to shed light on this hypothesis is to compare hippocampal transcriptome of mice treated with vehicle to those acutely treated with SAHA, either by DNA microarray or by RNA-Seq.

\section{Summary and Future Directions}

In the first part of my work I characterized the transgenic mouse line that had a gene coding for histone methyltransferase, Mll2, knocked-out in forebrain excitatory neurons, in order to gain a closer understanding of the role of histone methylation in behaviour and cognition. The knock-out mice had increased anxiety and impaired learning assessed by several paradigms. This was accompanied by extensive downregulation of gene expression in dorsal dentate gyrus but not in dorsal CA. Many of downregulated genes code for proteins that play role in pathways/mechanisms involved in synaptic signalling and plasticity. Downregulation of gene expression as a result of Mll2 knock-down was also accompanied by a decrease in Histone 3 Lysine 4 tri and dimethylation, but not monomethylation, at gene promoters. Together with several other recent studies (Schaefer et al., 2009; Balemans et al., 2010; Jiang et al., 2010; Gupta et al., 2010) 
findings of this study will make a profound contribution to understanding the role of histone methylation in behaviour and learning \& memory in particular. One of the further experiments that is currently being performed on M112 forebrain-specific conditional knock-out mice are analysing gene expression in ventral dentate gyrus and CA by RNASeq in order to elucidate any specific effect that Ml12 knock-down may have on ventral hippocampus differently from the dorsal part of it. Another experiment that will soon be performed is analysing if acute SAHA injection leads to an increase in histone acetylation at gene promoters, downregulated as a result of Mll2 knock-down, in control and in F/F CKII mice (see 'Discussion').

M112 is not the only H3K4 methyltransferase in mammalian cells (see 'Introduction'). Therefore in order to gain a deeper and more complete understanding of the role of histone methylation in learning and memory the involvement of other methyltransferases in learning and memory should be investigated. And one further step that I am currently making in this direction is characterizing Mll1 F/F CKII mice. These mice also show learning impairment in fear conditioning and water maze paradigms. However, differently from Mll2 F/F CKII mice they do not show impairment in novel object recognition, and in contrast to Mll2 conditional knock-out mice they tend to show a decrease in anxiety behaviour. The molecular analysis of Mll1 F/F CKII mice is currently underway. 


\section{PART 2: EFFECTS OF DIFFERENT DURATIONS OF ENVIRONMENTAL ENRICHMENT ON LEARNING AND MEMORY}

\section{A certain minimum duration of environmental enrichment is required to facilitate learning}

Although a plethora of studies exist documenting the beneficial effect of environmental enrichment on learning \& memory, yet there exists no standardized protocol by which mice can be provided an enriched environment in different laboratories. As a result enriched environments provided to mice in different laboratories could differ from each other in their level of stimulation. Therefore before proceeding further with enrichment experiments it is always necessary to find out the duration that is enough to facilitate learning in given laboratory conditions. I found out that in our conditions 1 week of environmental enrichment was not yet enough to facilitate learning whereas 2 weeks of EE already were. This indicates that providing a novel and stimulating environment per se is not enough to facilitate learning. It has to be provided continuously for a certain amount of time. In the future it would be interesting also to find out how different components of enrichment affect the outcome. For example, in this study the mice were provided new toys everyday, and also everyday the existing toys were rearranged inside the cage (see 'Materials and Methods'). It would therefore be interesting to see to what extent changing and rearranging the toys contributes to the positive effects of enrichment on cognitive function. Would 2 weeks of EE still be enough to facilitate learning if during this time period the toys were not changed at all? Or changed only once, twice? And so on... Or, will environmental enrichment be effective at all without providing the novelty? Another interesting question that can be addressed is what roles physical and cognitive components of enrichment play on memory enhancement. Would providing mice only with toys without running wheels attenuate the beneficial effect of enrichment? These are all interesting questions that can be addressed in future studies. 


\section{The effects of long-term enrichment are persistent whereas those of short-term enrichment fade away soon after enrichment ceases}

Although, as mentioned above, a lot of studies exist documenting the beneficial role of environmental enrichment on cognition the number of studies investigating the persistence of its effects once enriched environment does not exist anymore are very scarce - almost non-existent. The only study known so far investigated the persistence of effect of EE on habituation to the open field (Amaral et al., 2008), where the authors showed that when enriched environment is presented for 8 weeks starting from weaning its effects persist up to at least 2 months after cessation of enrichment. However, this does not happen when mice are enriched for 4 weeks starting from weaning or from the tenth week of age (P70). Nevertheless, in my mind this study was incomplete since they did not test the persistence of effects of 8 weeks of EE starting from P70. Moreover, what interests me more is the effect of environmental enrichment on learning \& memory, which has not been investigated in that study. Furthermore, it would be highly beneficial for therapeutical purposes if the beneficial effects of environmental enrichment on cognition were persistent even when it is provided at adulthood. It would also be highly interesting for purely theoretical purposes to find out whether a living being, be it mouse or human, remains smart even after the enriched environment to which it was previously subjected to is removed. Therefore I set out to investigate this.

My first finding was that although 2 weeks of EE are enough to facilitate cognition, the effect of barely lasts up to 1 week. Mice that were enriched for 2 weeks and then kept in standard home cages for an additional 1 week did not have improved memory in fear conditioning test anymore compared to their control counterparts. However, in water maze test the learning-facilitating effects of EE persisted at least up to 1 week after cessation of enrichment - i.e. the mice that underwent 2 weeks of EE and then were kept in standard home cages for an additional 1week still managed to perform better in the water maze task compared to the ones which were kept in standard housing all the time. Next, I wondered to what extent the facilitated learning performance in the water maze task will persist in mice enriched for 2 weeks - i.e. whether the mice will remain smart for a much longer period of time after cessation of enrichment. In order to figure that out 
I kept the same mice in home cages for an additional 2 months. After that they were subjected to a modified water maze test, in which the platform was removed to another place and the visual cues around the water maze were rearranged. These were done in order to ensure that the mice are subjected to a completely novel learning test without any possible interference from the memory acquired in the original water maze test. On the first training day mice that were previously enriched and the ones always kept in home cages had similar escape latencies, from which it can be concluded that the enhanced memory that the enriched mice acquired in the original water maze task did not interfere with the current performance. It was observed that when kept in home cages for a much longer time (i.e. 2 months), mice that were enriched for 2 weeks do not perform better in the memory task than their control counterparts anymore. This indicates that the effects of short-term enrichment are not really persistent and fade away soon after enrichment ceases.

The next question to be addressed was whether the effects of enrichment on learning \& memory could be persistent if it is applied for a much longer duration. In other words, is environmental enrichment at all able to exert long-lasting effects on cognition? To find this out a group of mice was enriched for 10 weeks and then kept in standard home cages before being subjected to fear conditioning and water maze experiments. Two other groups included mice that were kept in home cages all the time and another one where mice were subjected to learning experiments immediately after 10 weeks of EE. First of all, differently from mice that underwent only 2 weeks of EE, mice that underwent 10 weeks of EE had increased memory in the fear conditioning task even after being kept in home cages for an additional 1 week. And as expected they also performed better than the ones continuously kept home cages in the water maze task as well. This indicates that when exerted for a much longer period, environmental enrichment is able to exert more persistent effects of cognition. However, still the crucial question was whether 10 weeks of EE would continue to exert its positive effects on learning \& memory for much longer time. Therefore, as in the experiment with mice enriched for 2 weeks, mice were kept in home cages for additional 2 months and then subjected to the modified water maze experiment explained above. Interestingly, they still had improved memory compared to the ones that were kept in home cages continuously. This indicates that environmental 
enrichment, when performed for a sufficiently long period of time does exert persistent effects on learning, which continue to last long after its cessation. This indeed is in line with findings of Amaral et al., where they demonstrated that mice enriched for 8 weeks starting from weaning continue to show facilitated habituation to the open field even 6 months after being kept in home cages. It is still likely that due to their more plastic brain juvenile mice may have a more facilitated response to environmental enrichment than the adult ones, and as a result enrichment performed at juvenile stage may have a stronger and probably more long-lasting effect which could last up to 6 months. However, given my findings that the effects of long-term enrichment in adult mice last at least up to 2 months it would still be interesting to see whether the adult mice enriched for 2-3 months would still have facilitated learning performance when tested even 6 months after being put back into standard home cages.

These findings strongly argue that there are qualitatively three levels of environmental enrichment; (i) the first level at which the amount of enrichment is still not enough to facilitate learning at all, (ii) the second level where the amount of enrichment is enough to facilitate learning but its effects fade away soon after its cessation, and (iii) the third level at which the beneficial effects of enrichment on learning last for a long-time after its cessation. This hierarchy strongly resembles the hierarchy that exists in synaptic plasticity; with simple synaptic transmission not being able to lead to any changes at the synapse, with stimulation of a certain strength leading to short-term changes at the synapse that account for early LTP which fades away quite soon after being initiated, and finally with stronger stimulation exerting a qualitatively different effect where changes at synapses undergo late LTP which lasts for much longer time (see 'Introduction' for details). It is interesting to note that the most important aspect that differentiates late LTP from early LTP and simple synaptic transmission is the involvement of novel gene expression in the former, and its absence in the latter two. Therefore, it was very tempting to speculate that the differences in the effects of different durations of environmental enrichment may lie in their differential ability to exert changes in gene expression - with enrichment of longer duration leading to changes in gene expression whereas enrichment of shorter duration not. 


\section{Long-term EE leads to substantial changes in gene expression whereas such changes are absent after short-term EE}

Hippocampal gene expression profile of mice kept in standard cages was compared to those that underwent 10 weeks of EE, by DNA microarray. It has been observed that 10 weeks of EE induced changes in expression of many genes (see the complete list in Appnedix 2). Quite a lot of them are involved in transcriptional activation, especially through histone acetylation. Some of them were upregulated (Cml5, Meoxl, Ing3) and some were downregulated (Klfl, Kat2a). This may indicate that environmental enrichment mobilizes some additional transcriptional machinery, which has been latent before, when responding to novel stimuli. The increase in the level of some transcription factors may not in turn lead to an additional wave of changes in gene expression after enrichment as long as the mice remain in the "naïve" state. However, those novel transcription factors may come into play and make a difference when the mice are subjected to a learning stimulus. Therefore, it would be interesting to compare gene expression changes after a learning stimulus (e.g. fear conditioning) in home caged mice and mice enriched for 10 weeks.

Another group of genes differentially regulated after enrichment and which also deserve special attention are chemokine ligands (Ccl8, Ccl6 and Ccl17). Chemokine ligands are

secretory molecules that are secreted from glial cells and act on neurons through binding to chemokine receptors. The involvement of chemokine signalling in synaptic plasticity, and in neuronal function and survival has been repeatedly documented (Vlkolinsky et al., 2004; Ben Achour and Pascual, 2010; Nicolai et al., 2010; Nelson et al., 2011). An interesting observation was that among the genes that were confirmed by qRT-PCR only the expression of Ccl8 persisted at the same level up to 2 months after cessation of enrichment. The expression of all the other genes went back to the basal (i.e. home cage) levels when the mice were kept in home cages for two months after 10 weeks of enrichment. This is a very intriguing finding, which deserves further study. It might be possible that persistently elevated levels of Ccl8 in the hippocampus may serve to facilitate synaptic plasticity even after the enriched environment is not provided anymore. The mechanism ensuring the persistence in the expression level of Ccl8 may in turn act 
through changes in DNA methylation at its promoter. DNA methylation is known to be a more persistent epigenetic mark than histone modifications (Day and Sweatt, 2010), and has also previously been shown to be responsible for long-term changes in behaviour even throughout generations (Miller and Sweatt, 2007; Lubin et al., 2008; Miller et al., 2008; Feng et al., 2010; Miller et al., 2010; Franklin et al., 2010; Franklin and Mansuy, 2010). Moreover, the fact that environmental enrichment induces an increase also in the expression of genes coding for proteins secreted from glial cells indicates that EE affects not only neurons but glial cells as well, and that the role of glial cells in learning \& memory improvement should not be disregarded.

Another group of genes differentially regulated after 10 weeks of EE are involved in cGMP signalling. The one which is upregulated is Tjp2, and it possesses guanylate kinase activity which in turn facilitates cGMP signalling pathway. Another one, which is downregulated, is Pde6h and codes for phosphodiesterase. Phosphodiesterases catalyze cGMP catabolism thereby inhibiting cGMP-signalling pathway. Indeed, application of phosphodiesterase inhibitors has been shown to facilitate LTP and improve memory (Barad et al., 1998; Navakkode et al., 2004), hence it is quite expected that environmental enrichment would facilitate learning by removing a brake on synaptic plasticity.

Two more genes that deserve attention are Paipl and Plbd1. Both of them are upregulated after 10 weeks of EE. The protein encoded by Paipl facilitates translational initiation. Apart from regulation of transcription, regulation of mRNA translation has also been shown to be involved in synaptic plasticity and LTP induction (Kelleher et al., 2004a; Kelleher et al., 2004b). Especially, regulation of mRNA translation at the dendrites and spines is known to be a crucial factor in synaptic plasticity (Kelleher et al., 2004a; Kelleher et al., 2004b). It is therefore highly likely that environmental enrichment facilitates learning not only through mediating changes in gene expression but also through regulating mRNA translation. The other gene is Plbdl and codes for a phospholipase. Phospholipases promote synaptic plasticity through cleavage of membrane phospholipids, and the cleavage products (i.e. lipid messengers) that occur as a result of phospholipase action act as second messengers in intracellular signalling 
cascades involved in LTP induction (Dumuis et al., 1988; Pontzer et al., 1990; Sanfeliu et al., 1990; Wolf et al., 1995; Hawthorne, 1996; Gottschalk et al., 1999; Yang et al., 2001).

However, the most intriguing finding was that 2 weeks of enrichment induced only mild changes in gene expression. Among 16 genes which have been confirmed in hippocampus of mice that underwent 10 weeks of EE only three were regulated in the same way after 2 weeks of EE. These were Pde6h, Plbdl and Klfl. However, in order to reach more general conclusions a DNA microarray study comparing hippocampal transcription profile of home caged mice and mice enriched for 2 weeks has to be performed. Nevertheless, even at this stage it can be fairly argued that one of the crucial differences between short-term and long-term enrichment is in the extent of gene expression changes that they can mediate. And this differential ability to induce changes in gene expression is most probably the main cause behind the qualitatively different outcomes that different durations of enrichment lead to. This in turn acts in favour of the hypothesis that induction of synaptic plasticity and the actions of environmental enrichment are exact phenocopies of each other, being governed by comparable processes.

\section{Summary and Future Directions}

In the second part of my PhD work I studied the effect of environmental enrichment (EE) on learning \& memory and different effects that different durations of EE exert. It turned out that the process of environmental enrichment can be broadly classified into three different stages: (i) duration of EE that is not enough to facilitate learning at all (e.g. 1 week), (ii) short-term EE that is enough to lead to learning enhancement but whose effects fade away soon after enrichment ceases, and (iii) long-term EE which is both enough to facilitate learning and also exert long-lasting effects which persist for a long time after its cessation. One mechanism underlying this difference in in the level of persistence between shot-term and long-term EE is the differential ability to lead to changes in gene expression - long-term EE leading to changes in expression of many 
genes whereas short-term EE resulting only in limited change in gene expression. One more interesting observation was that among the genes confirmed by qRT-PCR (16 genes) only Ccl8 was shown to retain its level of expression induced by EE even after cessation of the latter. Further analyses need to be performed in order to elucidate the mechanism(s) mediating the persistent expression of $C c l 8$ gene - DNA methylation being the strongest candidate.

One further interesting question that may be addressed is whether the memory enhancement mediated by short-term and long-term enrichment depends on the same mechanisms in the first place. In other words, given the obvious difference in the level of persistence of the effects of those two kinds of enrichment it would be wise to ask whether the learning enhancement itself mediated by short-term enrichment is mechanistically different from that mediated by long-term enrichment - are these two "learning enhancements" of a different type in the first place. Therefore, an interesting experiment would be to compare the changes in hippocampal gene expression in "Home Cage", "2 weeks EE" and "10 weeks EE" mice after fear conditioning. It has previously been shown that fear conditioning leads to profound changes in expression of many genes involved in synaptic plasticity (Peleg et al., 2010). Hence it would be interesting to see whether short-term and long-term enrichment would lead to a different response to fear conditioning in terms gene expression - different from home caged mice and also different from each other. 


\section{REFERENCES}

Abeliovich, A., R. Paylor, et al. (1993). "PKC gamma mutant mice exhibit mild deficits in spatial and contextual learning." Cell 75(7): 1263-1271.

Ahn, H. J., C. M. Hernandez, et al. (2008). "c-Rel, an NF-kappaB family transcription factor, is required for hippocampal long-term synaptic plasticity and memory formation." Learning \& memory 15(7): 539-549.

Akazawa, C., M. Ishibashi, et al. (1995). "A mammalian helix-loop-helix factor structurally related to the product of Drosophila proneural gene atonal is a positive transcriptional regulator expressed in the developing nervous system." The Journal of biological chemistry 270(15): 8730-8738.

Alarcon, J. M., G. Malleret, et al. (2004). "Chromatin acetylation, memory, and LTP are impaired in $\mathrm{CBP}+/-$ mice: a model for the cognitive deficit in Rubinstein-Taybi syndrome and its amelioration." Neuron 42(6): 947-959.

Albensi, B. C. and M. P. Mattson (2000). "Evidence for the involvement of TNF and NF-kappaB in hippocampal synaptic plasticity." Synapse 35(2): 151-159.

Alkon, D. L., J. V. Sanchez-Andres, et al. (1992). "Long-term transformation of an inhibitory into an excitatory GABAergic synaptic response." Proceedings of the National Academy of Sciences of the United States of America 89(24): 11862-11866.

Amaral, O. B., R. S. Vargas, et al. (2008). "Duration of environmental enrichment influences the magnitude and persistence of its behavioral effects on mice." Physiology \& behavior 93(1-2): 388394.

Andreu-Vieyra, C. V., R. Chen, et al. (2010). "MLL2 is required in oocytes for bulk histone 3 lysine 4 trimethylation and transcriptional silencing." PLoS biology 8(8).

Ansari, K. I. and S. S. Mandal (2010). "Mixed lineage leukemia: roles in gene expression, hormone signaling and mRNA processing." The FEBS journal 277(8): 1790-1804.

Ansari, K. I., B. P. Mishra, et al. (2008). "Human CpG binding protein interacts with MLL1, MLL2 and hSet1 and regulates Hox gene expression." Biochimica et biophysica acta 1779(1): 66-73.

Artola, A., J. C. von Frijtag, et al. (2006). "Long-lasting modulation of the induction of LTD and LTP in rat hippocampal CA1 by behavioural stress and environmental enrichment." The European journal of neuroscience 23(1): 261-272. 
Atkins, C. M., J. C. Selcher, et al. (1998). "The MAPK cascade is required for mammalian associative learning." Nature neuroscience 1(7): 602-609.

Baddeley, A. (2003). "Working memory: looking back and looking forward." Nature reviews. Neuroscience 4(10): 829-839.

Bailey, C. H. and M. Chen (1983). "Morphological basis of long-term habituation and sensitization in Aplysia." Science 220(4592): 91-93.

Bailey, C. H. and E. R. Kandel (1993). "Structural changes accompanying memory storage." Annual review of physiology 55: 397-426.

Balemans, M. C., M. M. Huibers, et al. (2010). "Reduced exploration, increased anxiety, and altered social behavior: Autistic-like features of euchromatin histone methyltransferase 1 heterozygous knockout mice." Behavioural brain research 208(1): 47-55.

Bannerman, D. M., R. M. Deacon, et al. (2004). "A comparison of GluR-A-deficient and wild-type mice on a test battery assessing sensorimotor, affective, and cognitive behaviors." Behavioral neuroscience 118(3): 643-647.

Bannerman, D. M., J. N. Rawlins, et al. (2004). "Regional dissociations within the hippocampus-memory and anxiety." Neuroscience and biobehavioral reviews 28(3): 273-283.

Bannister, A. J. and T. Kouzarides (2004). "Histone methylation: recognizing the methyl mark." Methods in enzymology 376: 269-288.

Barad, M., R. Bourtchouladze, et al. (1998). "Rolipram, a type IV-specific phosphodiesterase inhibitor, facilitates the establishment of long-lasting long-term potentiation and improves memory." Proceedings of the National Academy of Sciences of the United States of America 95(25): 1502015025 .

Barco, A., C. H. Bailey, et al. (2006). "Common molecular mechanisms in explicit and implicit memory." Journal of neurochemistry 97(6): 1520-1533.

Becker, J. T. and R. G. Morris (1999). "Working memory(s)." Brain and cognition 41(1): 1-8.

Bell, O., C. Wirbelauer, et al. (2007). "Localized H3K36 methylation states define histone H4K16 acetylation during transcriptional elongation in Drosophila." The EMBO journal 26(24): 4974-4984.

Ben Achour, S. and O. Pascual (2010). "Glia: the many ways to modulate synaptic plasticity." Neurochemistry international 57(4): 440-445. 
Benaroya-Milshtein, N., N. Hollander, et al. (2004). "Environmental enrichment in mice decreases anxiety, attenuates stress responses and enhances natural killer cell activity." The European journal of neuroscience 20(5): 1341-1347.

Benjamini, Y. and Y. Hochberg (1995). "Controlling the False Discovery Rate - a Practical and Powerful Approach to Multiple Testing." Journal of the Royal Statistical Society Series BMethodological 57(1): 289-300.

Bennett, E. L., M. R. Rosenzweig, et al. (1969). "Rat brain: effects of environmental enrichment on wet and dry weights." Science 163(3869): 825-826.

Bennett, J. C., P. A. McRae, et al. (2006). "Long-term continuous, but not daily, environmental enrichment reduces spatial memory decline in aged male mice." Neurobiology of learning and memory 85(2): 139-152.

Bergado, J. A., W. Almaguer-Melian, et al. (2003). "Behavioral reinforcement of long-term potentiation in rat dentate gyrus in vivo is protein synthesis-dependent." Neuroscience letters 351(1): $56-58$.

Bertoglio, L. J., S. R. Joca, et al. (2006). "Further evidence that anxiety and memory are regionally dissociated within the hippocampus." Behavioural brain research 175(1): 183-188.

Bhalla, U. S. and R. Iyengar (1999). "Emergent properties of networks of biological signaling pathways." Science 283(5400): 381-387.

Bhalla, U. S., P. T. Ram, et al. (2002). "MAP kinase phosphatase as a locus of flexibility in a mitogenactivated protein kinase signaling network." Science 297(5583): 1018-1023.

Bhaumik, S. R., E. Smith, et al. (2007). "Covalent modifications of histones during development and disease pathogenesis." Nature structural \& molecular biology 14(11): 1008-1016.

Bierman, E. J., H. C. Comijs, et al. (2005). "Effects of anxiety versus depression on cognition in later life." The American journal of geriatric psychiatry : official journal of the American Association for Geriatric Psychiatry 13(8): 686-693.

Bishop, S. J. (2007). "Neurocognitive mechanisms of anxiety: an integrative account." Trends in cognitive sciences 11(7): 307-316.

Bito, H., K. Deisseroth, et al. (1996). "CREB phosphorylation and dephosphorylation: a Ca(2+)- and stimulus duration-dependent switch for hippocampal gene expression." Cell 87(7): 1203-1214. 
Blackstone, C., T. H. Murphy, et al. (1994). "Cyclic AMP and synaptic activity-dependent phosphorylation of AMPA-preferring glutamate receptors." The Journal of neuroscience : the official journal of the Society for Neuroscience 14(12): 7585-7593.

Blanchard, D. C., N. S. Canteras, et al. (2005). "Lesions of structures showing FOS expression to cat presentation: effects on responsivity to a Cat, Cat odor, and nonpredator threat." Neuroscience and biobehavioral reviews 29(8): 1243-1253.

Blanchard, D. C., C. I. Li, et al. (2003). "Dorsal premammillary nucleus differentially modulates defensive behaviors induced by different threat stimuli in rats." Neuroscience letters 345(3): 145-148.

Blanchard, R. J. and D. C. Blanchard (1969). "Passive and active reactions to fear-eliciting stimuli." Journal of comparative and physiological psychology 68(1): 129-135.

Bliss, T. V. P. and T. Lomo (1973). "Long-Lasting Potentiation of Synaptic Transmission in Dentate Area of Anesthetized Rabbit Following Stimulation of Perforant Path." Journal of Physiology-London 232(2): 331-356.

Blitzer, R. D., T. Wong, et al. (1995). "Postsynaptic cAMP pathway gates early LTP in hippocampal CA1 region." Neuron 15(6): 1403-1414.

Bontekoe, C. J., K. L. Mcllwain, et al. (2002). "Knockout mouse model for Fxr2: a model for mental retardation." Human molecular genetics 11(5): 487-498.

Bourtchuladze, R., B. Frenguelli, et al. (1994). "Deficient long-term memory in mice with a targeted mutation of the cAMP-responsive element-binding protein." Cell 79(1): 59-68.

Bowers, B. J., A. C. Collins, et al. (2000). "Mice lacking PKC gamma exhibit decreased anxiety." Behavior genetics 30(2): 111-121.

Boyer, L. A., K. Plath, et al. (2006). "Polycomb complexes repress developmental regulators in murine embryonic stem cells." Nature 441(7091): 349-353.

Bradler, J. E. and G. Barrioneuvo (1989). "Long-term potentiation in hippocampal CA3 neurons: tetanized input regulates heterosynaptic efficacy." Synapse 4(2): 132-142.

Brami-Cherrier, K., J. Lavaur, et al. (2007). "Glutamate induces histone H3 phosphorylation but not acetylation in striatal neurons: role of mitogen- and stress-activated kinase-1." Journal of neurochemistry 101(3): 697-708.

Brami-Cherrier, K., E. Roze, et al. (2009). "Role of the ERK/MSK1 signalling pathway in chromatin remodelling and brain responses to drugs of abuse." Journal of neurochemistry 108(6): 1323-1335. 
Bredy, T. W., H. Wu, et al. (2007). "Histone modifications around individual BDNF gene promoters in prefrontal cortex are associated with extinction of conditioned fear." Learning \& memory 14(4): 268-276.

Bruel-Jungerman, E., S. Laroche, et al. (2005). "New neurons in the dentate gyrus are involved in the expression of enhanced long-term memory following environmental enrichment." The European journal of neuroscience 21(2): 513-521.

Buhry, L., A. H. Azizi, et al. (2011). "Reactivation, replay, and preplay: how it might all fit together." Neural plasticity 2011: 203462.

Caceres, A., J. Mautino, et al. (1992). "Suppression of MAP2 in cultured cerebellar macroneurons inhibits minor neurite formation." Neuron 9(4): 607-618.

Cai, F., F. Wang, et al. (2008). "Redox modulation of long-term potentiation in the hippocampus via regulation of the glycogen synthase kinase-3beta pathway." Free radical biology \& medicine 45(7): 964-970.

Calabresi, P., A. Pisani, et al. (1997). "Synaptic plasticity and physiological interactions between dopamine and glutamate in the striatum." Neuroscience and biobehavioral reviews 21(4): 519-523.

Calabresi, P., A. Pisani, et al. (1992). "Long-term Potentiation in the Striatum is Unmasked by Removing the Voltage-dependent Magnesium Block of NMDA Receptor Channels." The European journal of neuroscience 4(10): 929-935.

Campeau, S. and M. Davis (1995). "Involvement of the central nucleus and basolateral complex of the amygdala in fear conditioning measured with fear-potentiated startle in rats trained concurrently with auditory and visual conditioned stimuli." The Journal of neuroscience : the official journal of the Society for Neuroscience 15(3 Pt 2): 2301-2311.

Canteras, N. S. (2002). "The medial hypothalamic defensive system: hodological organization and functional implications." Pharmacology, biochemistry, and behavior 71(3): 481-491.

Canteras, N. S., S. Chiavegatto, et al. (1997). "Severe reduction of rat defensive behavior to a predator by discrete hypothalamic chemical lesions." Brain Research Bulletin 44(3): 297-305.

Canteras, N. S., L. B. Resstel, et al. (2010). "Neuroanatomy of anxiety." Current topics in behavioral neurosciences 2: 77-96.

Carvalho, A. L., C. B. Duarte, et al. (2000). "Regulation of AMPA receptors by phosphorylation." Neurochemical research 25(9-10): 1245-1255. 
Castellucci, V. F., W. N. Frost, et al. (1986). "Cell and molecular analysis of long-term sensitization in Aplysia." Journal de physiologie 81(4): 349-357.

Charbonneau, H. and N. K. Tonks (1992). "1002 protein phosphatases?" Annual review of cell biology 8: 463-493.

Charles, C. H., H. Sun, et al. (1993). "The growth factor-inducible immediate-early gene 3CH134 encodes a protein-tyrosine-phosphatase." Proceedings of the National Academy of Sciences of the United States of America 90(11): 5292-5296.

Charpier, S. and J. M. Deniau (1997). "In vivo activity-dependent plasticity at cortico-striatal connections: evidence for physiological long-term potentiation." Proceedings of the National Academy of Sciences of the United States of America 94(13): 7036-7040.

Chwang, W. B., J. S. Arthur, et al. (2007). "The nuclear kinase mitogen- and stress-activated protein kinase 1 regulates hippocampal chromatin remodeling in memory formation." The Journal of neuroscience : the official journal of the Society for Neuroscience 27(46): 12732-12742.

Chwang, W. B., K. J. O'Riordan, et al. (2006). "ERK/MAPK regulates hippocampal histone phosphorylation following contextual fear conditioning." Learning \& memory 13(3): 322-328.

Clinton, M., J. Manson, et al. (2000). "Gene expression changes during murine postnatal brain development." Genome biology 1(3): RESEARCH0005.

Collin, C., W. A. Devane, et al. (1995). "Long-term synaptic transformation of hippocampal CA1 gamma-aminobutyric acid synapses and the effect of anandamide." Proceedings of the National Academy of Sciences of the United States of America 92(22): 10167-10171.

Contarino, A., F. Dellu, et al. (1999). "Reduced anxiety-like and cognitive performance in mice lacking the corticotropin-releasing factor receptor 1." Brain research 835(1): 1-9.

Cooper-Kuhn, C. M., M. Vroemen, et al. (2002). "Impaired adult neurogenesis in mice lacking the transcription factor E2F1." Molecular and cellular neurosciences 21(2): 312-323.

Corkin, S. (2002). "What's new with the amnesic patient H.M.?" Nature reviews. Neuroscience 3(2): 153-160.

Coutureau, E., R. Galani, et al. (2000). "Selective lesions of the entorhinal cortex, the hippocampus, or the fimbria-fornix in rats: a comparison of effects on spontaneous and amphetamine-induced locomotion." Experimental brain research. Experimentelle Hirnforschung. Experimentation cerebrale 131(3): 381-392. 
Cowan, N. (2001). "The magical number 4 in short-term memory: a reconsideration of mental storage capacity." The Behavioral and brain sciences 24(1): 87-114; discussion 114-185.

Crepel, F. and D. Jaillard (1991). "Pairing of pre- and postsynaptic activities in cerebellar Purkinje cells induces long-term changes in synaptic efficacy in vitro." The Journal of physiology 432: 123141.

Crusio, W. E. and H. Schwegler (2005). "Learning spatial orientation tasks in the radial-maze and structural variation in the hippocampus in inbred mice." Behavioral and brain functions : BBF 1(1): 3 .

D'Adamo, P., D. P. Wolfer, et al. (2004). "Mice deficient for the synaptic vesicle protein Rab3a show impaired spatial reversal learning and increased explorative activity but none of the behavioral changes shown by mice deficient for the Rab3a regulator Gdi1." The European journal of neuroscience 19(7): 1895-1905.

D'Angelo, E., P. Rossi, et al. (1999). "Evidence for NMDA and mGlu receptor-dependent long-term potentiation of mossy fiber-granule cell transmission in rat cerebellum." Journal of neurophysiology 81(1): 277-287.

Dale, N., S. Schacher, et al. (1988). "Long-term facilitation in Aplysia involves increase in transmitter release." Science 239(4837): 282-285.

Davis, S., P. Vanhoutte, et al. (2000). "The MAPK/ERK cascade targets both Elk-1 and cAMP response element-binding protein to control long-term potentiation-dependent gene expression in the dentate gyrus in vivo." The Journal of neuroscience : the official journal of the Society for Neuroscience 20(12): 4563-4572.

Day, J. J. and J. D. Sweatt (2010). "DNA methylation and memory formation." Nature neuroscience 13(11): 1319-1323.

Degroot, A. and D. Treit (2004). "Anxiety is functionally segregated within the septo-hippocampal system." Brain research 1001(1-2): 60-71.

Deisseroth, K., H. Bito, et al. (1996). "Signaling from synapse to nucleus: postsynaptic CREB phosphorylation during multiple forms of hippocampal synaptic plasticity." Neuron 16(1): 89-101.

Delanoy, R. L., D. L. Tucci, et al. (1983). "Amphetamine effects on long term potentiation in dentate granule cells." Pharmacology, biochemistry, and behavior 18(1): 137-139.

Demers, C., C. P. Chaturvedi, et al. (2007). "Activator-mediated recruitment of the MLL2 methyltransferase complex to the beta-globin locus." Molecular cell 27(4): 573-584. 
Diamond, M. C., C. A. Ingham, et al. (1976). "Effects of environment on morphology of rat cerebral cortex and hippocampus." Journal of neurobiology 7(1): 75-85.

Diamond, M. C., M. R. Rosenzweig, et al. (1972). "Effects of environmental enrichment and impoverishment on rat cerebral cortex." Journal of neurobiology 3(1): 47-64.

Dielenberg, R. A., G. E. Hunt, et al. (2001). "'When a rat smells a cat': The distribution of Fos immunoreactivity in rat brain following exposure to a predatory odor." Neuroscience 104(4): 10851097.

Dirks, A., T. Pattij, et al. (2001). "5-HT1B receptor knockout, but not 5-HT1A receptor knockout mice, show reduced startle reactivity and footshock-induced sensitization, as measured with the acoustic startle response." Behavioural brain research 118(2): 169-178.

Donaldson, W. and E. Tulving "Organization of memory." Isbn 0-12-703650-4. 1972. Academic Press, New York. Xiii + 423 P. Illus. Ref.

Dong, H. W., L. W. Swanson, et al. (2009). "Genomic-anatomic evidence for distinct functional domains in hippocampal field CA1." Proceedings of the National Academy of Sciences of the United States of America 106(28): 11794-11799.

Dou, Y., T. A. Milne, et al. (2006). "Regulation of MLL1 H3K4 methyltransferase activity by its core components." Nature structural \& molecular biology 13(8): 713-719.

Dou, Y., T. A. Milne, et al. (2005). "Physical association and coordinate function of the H3 K4 methyltransferase MLL1 and the H4 K16 acetyltransferase MOF." Cell 121(6): 873-885.

Dreijerink, K. M., K. W. Mulder, et al. (2006). "Menin links estrogen receptor activation to histone H3K4 trimethylation." Cancer research 66(9): 4929-4935.

Duffy, S. N., K. J. Craddock, et al. (2001). "Environmental enrichment modifies the PKA-dependence of hippocampal LTP and improves hippocampus-dependent memory." Learning \& memory 8(1): 2634.

Dumuis, A., M. Sebben, et al. (1988). "NMDA receptors activate the arachidonic acid cascade system in striatal neurons." Nature 336(6194): 68-70.

Durand, M., A. Kolpak, et al. (2007). "The OXR domain defines a conserved family of eukaryotic oxidation resistance proteins." BMC cell biology 8: 13.

Ehninger, D., W. Li, et al. (2008). "Reversing neurodevelopmental disorders in adults." Neuron 60(6): 950-960.

Eichenbaum, H. (1997). "How does the brain organize memories?" Science 277(5324): 330-332 
Eichenbaum, H. (2001). "The hippocampus and declarative memory: cognitive mechanisms and neural codes." Behavioural brain research 127(1-2): 199-207.

El Hage, W., G. Griebel, et al. (2006). "Long-term impaired memory following predatory stress in mice." Physiology \& behavior 87(1): 45-50.

El Hage, W., S. Peronny, et al. (2004). "Impaired memory following predatory stress in mice is improved by fluoxetine." Progress in neuro-psychopharmacology \& biological psychiatry 28(1): 123128.

Elliott, N. A. and M. R. Volkert (2004). "Stress induction and mitochondrial localization of Oxr1 proteins in yeast and humans." Molecular and cellular biology 24(8): 3180-3187.

English, J. D. and J. D. Sweatt (1996). "Activation of p42 mitogen-activated protein kinase in hippocampal long term potentiation." The Journal of biological chemistry 271(40): 24329-24332.

English, J. D. and J. D. Sweatt (1997). "A requirement for the mitogen-activated protein kinase cascade in hippocampal long term potentiation." The Journal of biological chemistry 272(31): 1910319106.

Etkin, A. and T. D. Wager (2007). "Functional neuroimaging of anxiety: A meta-analysis of emotional processing in PTSD, social anxiety disorder, and specific phobia." American Journal of Psychiatry 164(10): 1476-1488.

Euston, D. R., M. Tatsuno, et al. (2007). "Fast-forward playback of recent memory sequences in prefrontal cortex during sleep." Science 318(5853): 1147-1150.

Faherty, C. J., D. Kerley, et al. (2003). "A Golgi-Cox morphological analysis of neuronal changes induced by environmental enrichment." Brain research. Developmental brain research 141(1-2): 5561.

Fanselow, M. S. and H. W. Dong (2010). "Are the dorsal and ventral hippocampus functionally distinct structures?" Neuron 65(1): 7-19.

Feng, J., Y. Zhou, et al. (2010). "Dnmt1 and Dnmt3a maintain DNA methylation and regulate synaptic function in adult forebrain neurons." Nature neuroscience 13(4): 423-430.

Fischer, A., F. Sananbenesi, et al. (2007). "Recovery of learning and memory is associated with chromatin remodelling." Nature 447(7141): 178-182.

Fitts, P. M. (1954). "The information capacity of the human motor system in controlling the amplitude of movement." Journal of Experimental Psychology 47: 381-391 
FitzGerald, K. T. and M. O. Diaz (1999). "MLL2: A new mammalian member of the trx/MLL family of genes." Genomics 59(2): 187-192.

Foster, T. C. and T. C. Dumas (2001). "Mechanism for increased hippocampal synaptic strength following differential experience." Journal of neurophysiology 85(4): 1377-1383.

Frankland, P. W. and B. Bontempi (2005). "The organization of recent and remote memories." Nature reviews. Neuroscience 6(2): 119-130.

Frankland, P. W., B. Bontempi, et al. (2004). "The involvement of the anterior cingulate cortex in remote contextual fear memory." Science 304(5672): 881-883.

Franklin, T. B. and I. M. Mansuy (2010). "Epigenetic inheritance in mammals: evidence for the impact of adverse environmental effects." Neurobiology of disease 39(1): 61-65.

Franklin, T. B., H. Russig, et al. (2010). "Epigenetic transmission of the impact of early stress across generations." Biological psychiatry 68(5): 408-415.

Freudenthal, R., A. Romano, et al. (2004). "Transcription factor NF-kappaB activation after in vivo perforant path LTP in mouse hippocampus." Hippocampus 14(6): 677-683.

Frey, U., Y. Y. Huang, et al. (1993). "Effects of cAMP simulate a late stage of LTP in hippocampal CA1 neurons." Science 260(5114): 1661-1664.

Frey, U., M. Krug, et al. (1988). "Anisomycin, an inhibitor of protein synthesis, blocks late phases of LTP phenomena in the hippocampal CA1 region in vitro." Brain research 452(1-2): 57-65.

Frey, U. and R. G. Morris (1997). "Synaptic tagging and long-term potentiation." Nature 385(6616): 533-536.

Frick, K. M. and S. M. Fernandez (2003). "Enrichment enhances spatial memory and increases synaptophysin levels in aged female mice." Neurobiology of aging 24(4): 615-626.

Gabbott, P. L., T. A. Warner, et al. (2005). "Prefrontal cortex in the rat: projections to subcortical autonomic, motor, and limbic centers." The Journal of comparative neurology 492(2): 145-177.

Gale, G. D., S. G. Anagnostaras, et al. (2004). "Role of the basolateral amygdala in the storage of fear memories across the adult lifetime of rats." The Journal of neuroscience : the official journal of the Society for Neuroscience 24(15): 3810-3815.

Gamblin, T. C., K. Nachmanoff, et al. (1996). "Recombinant microtubule-associated protein 2c reduces the dynamic instability of individual microtubules." Biochemistry 35(38): 12576-12586. 
Glaser, S., S. Lubitz, et al. (2009). "The histone 3 lysine 4 methyltransferase, Mll2, is only required briefly in development and spermatogenesis." Epigenetics \& chromatin 2(1): 5.

Glaser, S., J. Schaft, et al. (2006). "Multiple epigenetic maintenance factors implicated by the loss of M112 in mouse development." Development 133(8): 1423-1432.

Gottschalk, W. A., H. Jiang, et al. (1999). "Signaling mechanisms mediating BDNF modulation of synaptic plasticity in the hippocampus." Learning \& memory 6(3): 243-256.

Gozlan, H., R. Khazipov, et al. (1995). "In CA1 hippocampal neurons, the redox state of NMDA receptors determines LTP expressed by NMDA but not by AMPA receptors." Journal of neurophysiology 73(6): 2612-2617.

Gray, J. A. and N. McNaughton (2000). "Neural anxiety systems: Relevant fault-lines to trace and treat disorders." European Journal of Neuroscience 12: 311-311.

Greco, B. and M. Carli (2006). "Reduced attention and increased impulsivity in mice lacking NPY Y2 receptors: relation to anxiolytic-like phenotype." Behavioural brain research 169(2): 325-334.

Greengard, P., J. Jen, et al. (1991). "Enhancement of the glutamate response by cAMP-dependent

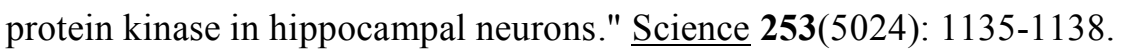

Greenough, W. T. and F. R. Volkmar (1973). "Pattern of dendritic branching in occipital cortex of rats reared in complex environments." Experimental neurology 40(2): 491-504.

Greenough, W. T., F. R. Volkmar, et al. (1973). "Effects of rearing complexity on dendritic branching in frontolateral and temporal cortex of the rat." Experimental neurology 41(2): 371-378.

Grewal, S. I. and D. Moazed (2003). "Heterochromatin and epigenetic control of gene expression." Science 301(5634): 798-802.

Guadano-Ferraz, A., R. Benavides-Piccione, et al. (2003). "Lack of thyroid hormone receptor alpha1 is associated with selective alterations in behavior and hippocampal circuits." $\underline{\text { Molecular psychiatry }} \mathbf{8}(1)$ : $30-38$.

Guan, J. S., S. J. Haggarty, et al. (2009). "HDAC2 negatively regulates memory formation and synaptic plasticity." Nature 459(7243): 55-60.

Gupta, S., S. Y. Kim, et al. (2010). "Histone methylation regulates memory formation." The Journal of neuroscience : the official journal of the Society for Neuroscience 30(10): 3589-3599.

Hack1, L. P. N. and A. P. Carobrez (2007). "Distinct ventral and dorsal hippocampus AP5 anxiolytic effects revealed in the elevated plus-maze task in rats." Neurobiology of Learning and Memory $\mathbf{8 8}(2)$ : 177-185. 
Hagihara, H., K. Toyama, et al. (2009). "Dissection of hippocampal dentate gyrus from adult mouse." Journal of visualized experiments : JoVE(33).

Harris, E. W., A. H. Ganong, et al. (1984). "Long-term potentiation in the hippocampus involves activation of N-methyl-D-aspartate receptors." Brain research 323(1): 132-137.

Hawthorne, J. N. (1996). "Phosphoinositides and synaptic transmission." Sub-cellular biochemistry 26: $43-57$.

Hayashi, Y. and A. K. Majewska (2005). "Dendritic spine geometry: functional implication and regulation." Neuron 46(4): 529-532.

Hebb, D.O. (1947). "The effects of early experience on problem solving at maturity." American Psychologist 2: 306-307

Henke, P. G. (1990). "Hippocampal pathway to the amygdala and stress ulcer development." Brain research bulletin 25(5): 691-695.

Hess, J. L., B. D. Yu, et al. (1997). "Defects in yolk sac hematopoiesis in Mll-null embryos." Blood 90(5): 1799-1806.

Higashima, M. and C. Yamamoto (1985). "Two components of long-term potentiation in mossy fiberinduced excitation in hippocampus." Experimental neurology 90(3): 529-539.

Horton, S. J. and O. Williams (2006). "The mechanism of hematopoietic progenitor cell immortalization by MLL-ENL." Cell cycle 5(4): 360-362.

Huang, Y. Y. and E. R. Kandel (1995). "D1/D5 receptor agonists induce a protein synthesis-dependent late potentiation in the CA1 region of the hippocampus." Proceedings of the National Academy of Sciences of the United States of America 92(7): 2446-2450.

Huber, R., M. F. Ghilardi, et al. (2004). "Local sleep and learning." Nature 430(6995): 78-81.

Huber, W., A. von Heydebreck, et al. (2002). "Variance stabilization applied to microarray data calibration and to the quantification of differential expression." Bioinformatics 18 Suppl 1: S96-104.

Hughes, C. M., O. Rozenblatt-Rosen, et al. (2004). "Menin associates with a trithorax family histone methyltransferase complex and with the hoxc8 locus." Molecular cell 13(4): 587-597.

Impey, S., M. Mark, et al. (1996). "Induction of CRE-mediated gene expression by stimuli that generate long-lasting LTP in area CA1 of the hippocampus." Neuron 16(5): 973-982.

Impey, S., D. M. Smith, et al. (1998). "Stimulation of cAMP response element (CRE)-mediated transcription during contextual learning." Nature neuroscience 1(7): 595-601. 
Izquierdo, I. and J. H. Medina (1995). "Correlation between the pharmacology of long-term potentiation and the pharmacology of memory." Neurobiology of learning and memory 63(1): 19-32.

Jaffe, D. and D. Johnston (1990). "Induction of long-term potentiation at hippocampal mossy-fiber synapses follows a Hebbian rule." Journal of neurophysiology 64(3): 948-960.

Jahn, R., W. Schiebler, et al. (1985). "A 38,000-dalton membrane protein (p38) present in synaptic vesicles." Proceedings of the National Academy of Sciences of the United States of America 82(12): 4137-4141.

Jaholkowski, P., A. Kiryk, et al. (2009). "New hippocampal neurons are not obligatory for memory formation; cyclin D2 knockout mice with no adult brain neurogenesis show learning." Learning \& memory 16(7): 439-451.

Ji, D. and M. A. Wilson (2007). "Coordinated memory replay in the visual cortex and hippocampus during sleep." Nature neuroscience 10(1): 100-107.

Jiang, Y., M. Jakovcevski, et al. (2010). "Setdb1 histone methyltransferase regulates mood-related behaviors and expression of the NMDA receptor subunit NR2B." The Journal of neuroscience : the official journal of the Society for Neuroscience 30(21): 7152-7167.

Johnson, G. V. and R. S. Jope (1992). "The role of microtubule-associated protein 2 (MAP-2) in neuronal growth, plasticity, and degeneration." Journal of neuroscience research 33(4): 505-512.

Jude, C. D., L. Climer, et al. (2007). "Unique and independent roles for MLL in adult hematopoietic stem cells and progenitors." Cell stem cell 1(3): 324-337.

Jung, M. W., S. I. Wiener, et al. (1994). "Comparison of spatial firing characteristics of units in dorsal and ventral hippocampus of the rat." The Journal of neuroscience : the official journal of the Society for Neuroscience 14(12): 7347-7356.

Justin, N., V. De Marco, et al. (2010). "Reading, writing and editing methylated lysines on histone tails: new insights from recent structural studies." Current opinion in structural biology 20(6): 730738.

Kaila, K., K. Lamsa, et al. (1997). "Long-lasting GABA-mediated depolarization evoked by highfrequency stimulation in pyramidal neurons of rat hippocampal slice is attributable to a networkdriven, bicarbonate-dependent $\mathrm{K}+$ transient." The Journal of neuroscience : the official journal of the Society for Neuroscience 17(20): 7662-7672.

Kalueff, A. V. (2007). "Neurobiology of memory and anxiety: from genes to behavior." Neural plasticity 2007: 78171. 
Kamiya, H., S. Sawada, et al. (1988). "Additive feature of long-term potentiation and phorbol esterinduced synaptic enhancement in the mossy fiber-CA3 synapse." Experimental neurology 102(3): 314317.

Kandel, E. R. (2001). "The molecular biology of memory storage: a dialogue between genes and synapses." Science 294(5544): 1030-1038.

Kelleher, R. J., 3rd, A. Govindarajan, et al. (2004). "Translational control by MAPK signaling in longterm synaptic plasticity and memory." Cell 116(3): 467-479.

Kelleher, R. J., 3rd, A. Govindarajan, et al. (2004). "Translational regulatory mechanisms in persistent forms of synaptic plasticity." Neuron 44(1): 59-73.

Keller, B. U., M. Hollmann, et al. (1992). "Calcium influx through subunits GluR1/GluR3 of kainate/AMPA receptor channels is regulated by cAMP dependent protein kinase." The EMBO journal 11(3): 891-896.

Kempermann, G., E. P. Brandon, et al. (1998). "Environmental stimulation of 129/SvJ mice causes increased cell proliferation and neurogenesis in the adult dentate gyrus." Current biology: CB 8(16): 939-942.

Kempermann, G., H. G. Kuhn, et al. (1997). "More hippocampal neurons in adult mice living in an enriched environment." Nature 386(6624): 493-495.

Kempermann, G., H. G. Kuhn, et al. (1998). "Experience-induced neurogenesis in the senescent dentate gyrus." The Journal of neuroscience : the official journal of the Society for Neuroscience 18(9): 3206-3212.

Kim, J. J. and M. S. Fanselow (1992). "Modality-specific retrograde amnesia of fear." $\underline{\text { Science }}$ 256(5057): 675-677.

Kim, S. Y., J. M. Levenson, et al. (2007). "Developmental regulation of Eed complex composition governs a switch in global histone modification in brain." The Journal of biological chemistry $\mathbf{2 8 2 ( 1 3 ) :}$ 9962-9972.

Kimura, S., S. Uchiyama, et al. (1998). "cAMP-dependent long-term potentiation of nitric oxide release from cerebellar parallel fibers in rats." The Journal of neuroscience : the official journal of the Society for Neuroscience 18(21): 8551-8558.

Kjelstrup, K. G., F. A. Tuvnes, et al. (2002). "Reduced fear expression after lesions of the ventral hippocampus." Proceedings of the National Academy of Sciences of the United States of America 99(16): 10825-10830. 
Klein, M., J. Camardo, et al. (1982). "Serotonin modulates a specific potassium current in the sensory neurons that show presynaptic facilitation in Aplysia." Proceedings of the National Academy of Sciences of the United States of America 79(18): 5713-5717.

Koshibu, K., J. Graff, et al. (2009). "Protein phosphatase 1 regulates the histone code for long-term memory." The Journal of neuroscience : the official journal of the Society for Neuroscience 29(41): 13079-13089.

Kouzarides, T. (2007). "Chromatin modifications and their function." Cell 128(4): 693-705.

Kreitzer, A. C. (2009). "Physiology and pharmacology of striatal neurons." Annual review of neuroscience 32: 127-147.

Krogan, N. J., J. Dover, et al. (2003). "The Pafl complex is required for histone H3 methylation by COMPASS and Dot1p: linking transcriptional elongation to histone methylation." Molecular cell 11(3): 721-729.

Kuczera, T., R. M. Stilling, et al. (2011). "The anaphase promoting complex is required for memory function in mice." Learning \& memory 18(1): 49-57.

Kudrimoti, H. S., C. A. Barnes, et al. (1999). "Reactivation of hippocampal cell assemblies: effects of behavioral state, experience, and EEG dynamics." The Journal of neuroscience : the official journal of the Society for Neuroscience 19(10): 4090-4101.

Kurdistani, S. K., S. Tavazoie, et al. (2004). "Mapping global histone acetylation patterns to gene expression." Cell 117(6): 721-733.

Kusuki, T., Y. Imahori, et al. (1997). "Dopaminergic modulation of LTP induction in the dentate gyrus of intact brain." Neuroreport 8(8): 2037-2040.

Lau, L. F. and D. Nathans (1985). "Identification of a set of genes expressed during the G0/G1 transition of cultured mouse cells." The EMBO journal 4(12): 3145-3151.

LeDoux, J. E. (1993). "Emotional memory: in search of systems and synapses." Annals of the New York Academy of Sciences 702: 149-157.

LeDoux, J. E. (1994). "Emotion, memory and the brain." Scientific American 270(6): 50-57.

LeDoux, J. E. (2000). "Emotion circuits in the brain." Annual review of neuroscience 23: 155-184.

Lee, E. H., W. L. Hsu, et al. (2003). "Enrichment enhances the expression of sgk, a glucocorticoidinduced gene, and facilitates spatial learning through glutamate AMPA receptor mediation." The European journal of neuroscience 18(10): 2842-2852. 
Lee, J. L., B. J. Everitt, et al. (2004). "Independent cellular processes for hippocampal memory consolidation and reconsolidation." Science 304(5672): 839-843.

Lee, J. S. and A. Shilatifard (2007). "A site to remember: H3K36 methylation a mark for histone deacetylation." Mutation research 618(1-2): 130-134.

Lee, T. I., R. G. Jenner, et al. (2006). "Control of developmental regulators by Polycomb in human embryonic stem cells." Cell 125(2): 301-313.

Leggio, M. G., L. Mandolesi, et al. (2005). "Environmental enrichment promotes improved spatial abilities and enhanced dendritic growth in the rat." Behavioural brain research 163(1): 78-90.

Levenson, J. M., K. J. O'Riordan, et al. (2004). "Regulation of histone acetylation during memory formation in the hippocampus." The Journal of biological chemistry 279(39): 40545-40559.

Li, B., M. Carey, et al. (2007). "The role of chromatin during transcription." Cell 128(4): 707-719.

Lim, R. W. and S. Halpain (2000). "Regulated association of microtubule-associated protein 2 (MAP2) with Src and Grb2: evidence for MAP2 as a scaffolding protein." The Journal of biological chemistry 275(27): 20578-20587.

Lu, Y. F., E. R. Kandel, et al. (1999). "Nitric oxide signaling contributes to late-phase LTP and CREB phosphorylation in the hippocampus." The Journal of neuroscience : the official journal of the Society for Neuroscience 19(23): 10250-10261.

Lubin, F. D., T. L. Roth, et al. (2008). "Epigenetic regulation of BDNF gene transcription in the consolidation of fear memory." The Journal of neuroscience : the official journal of the Society for Neuroscience 28(42): 10576-10586.

Lubin, F. D. and J. D. Sweatt (2007). "The IkappaB kinase regulates chromatin structure during reconsolidation of conditioned fear memories." Neuron 55(6): 942-957.

Malenka, R. C. (1991). "The role of postsynaptic calcium in the induction of long-term potentiation." Molecular neurobiology 5(2-4): 289-295.

Malenka, R. C. and M. F. Bear (2004). "LTP and LTD: an embarrassment of riches." Neuron 44(1): 521.

Malenka, R. C. and R. A. Nicoll (1993). "NMDA-receptor-dependent synaptic plasticity: multiple forms and mechanisms." Trends in neurosciences 16(12): 521-527. 
Malik, S. and S. R. Bhaumik (2010). "Mixed lineage leukemia: histone H3 lysine 4 methyltransferases from yeast to human." The FEBS journal 277(8): 1805-1821.

Malinow, R., Z. F. Mainen, et al. (2000). "LTP mechanisms: from silence to four-lane traffic." Current Opinion in Neurobiology 10(3): 352-357.

Malinow, R. and R. C. Malenka (2002). "AMPA receptor trafficking and synaptic plasticity." Annual review of neuroscience 25: 103-126.

Maquet, P., S. Laureys, et al. (2000). "Experience-dependent changes in cerebral activation during human REM sleep." Nature neuroscience 3(8): 831-836.

Maren, S. and M. S. Fanselow (1995). "Synaptic plasticity in the basolateral amygdala induced by hippocampal formation stimulation in vivo." The Journal of neuroscience : the official journal of the Society for Neuroscience 15(11): 7548-7564.

Markham, C. M., D. C. Blanchard, et al. (2004). "Modulation of predatory odor processing following lesions to the dorsal premammillary nucleus." Neuroscience letters 372(1-2): 22-26.

Marschalek, R. (2010). "Mixed lineage leukemia: roles in human malignancies and potential therapy." The FEBS journal 277(8): 1822-1831.

Martin, S. J., P. D. Grimwood, et al. (2000). "Synaptic plasticity and memory: an evaluation of the hypothesis." Annual review of neuroscience 23: 649-711.

Masugi, M., M. Yokoi, et al. (1999). "Metabotropic glutamate receptor subtype 7 ablation causes deficit in fear response and conditioned taste aversion." The Journal of neuroscience : the official journal of the Society for Neuroscience 19(3): 955-963.

Matsuzaki, M., N. Honkura, et al. (2004). "Structural basis of long-term potentiation in single dendritic spines." Nature 429(6993): 761-766.

Maviel, T., T. P. Durkin, et al. (2004). "Sites of neocortical reorganization critical for remote spatial memory." Science 305(5680): 96-99.

Mayford, M., M. E. Bach, et al. (1996). "Control of memory formation through regulated expression of a CaMKII transgene." Science 274(5293): 1678-1683.

Mayford, M., M. E. Bach, et al. (1996). "CaMKII function in the nervous system explored from a genetic perspective." Cold Spring Harbor symposia on quantitative biology 61: 219-224. 
McMahon, H. T., V. Y. Bolshakov, et al. (1996). "Synaptophysin, a major synaptic vesicle protein, is not essential for neurotransmitter release." Proceedings of the National Academy of Sciences of the United States of America 93(10): 4760-4764.

McMahon, K. A., S. Y. Hiew, et al. (2007). "Mll has a critical role in fetal and adult hematopoietic stem cell self-renewal." Cell stem cell 1(3): 338-345.

McQuown, S. C., R. M. Barrett, et al. (2011). "HDAC3 is a critical negative regulator of long-term memory formation." The Journal of neuroscience : the official journal of the Society for Neuroscience 31(2): 764-774.

Meberg, P. J., W. R. Kinney, et al. (1996). "Gene expression of the transcription factor NF-kappa B in hippocampus: regulation by synaptic activity." Brain research. Molecular brain research 38(2): 179190.

Meshi, D., M. R. Drew, et al. (2006). "Hippocampal neurogenesis is not required for behavioral effects of environmental enrichment." Nature neuroscience 9(6): 729-731.

Meyer, C., E. Kowarz, et al. (2009). "New insights to the MLL recombinome of acute leukemias." Leukemia : official journal of the Leukemia Society of America, Leukemia Research Fund, U.K 23(8): 1490-1499.

Miller, C. A., S. L. Campbell, et al. (2008). "DNA methylation and histone acetylation work in concert to regulate memory formation and synaptic plasticity." Neurobiology of learning and memory 89(4): 599-603.

Miller, C. A., C. F. Gavin, et al. (2010). "Cortical DNA methylation maintains remote memory." Nature neuroscience 13(6): 664-666.

Miller, C. A. and J. D. Sweatt (2007). "Covalent modification of DNA regulates memory formation." Neuron 53(6): 857-869.

Miller, T., N. J. Krogan, et al. (2001). "COMPASS: a complex of proteins associated with a trithoraxrelated SET domain protein." Proceedings of the National Academy of Sciences of the United States of America 98(23): 12902-12907.

Minichiello, L., M. Korte, et al. (1999). "Essential role for TrkB receptors in hippocampus-mediated learning." Neuron 24(2): 401-414.

Mo, R., S. M. Rao, et al. (2006). "Identification of the MLL2 complex as a coactivator for estrogen receptor alpha." The Journal of biological chemistry 281(23): 15714-15720. 
Mochida, S. and B. Libet (1990). "Postsynaptic long-term enhancement (LTE) by dopamine may be mediated by Ca2+ and calmodulin." Brain research 513(1): 144-148.

Morris, R. G. M. (1981). "Spatial Localization Does Not Require the Presence of Local Cues." Learning and Motivation 12(2): 239-260.

Moser, M. B. and E. I. Moser (1998). "Functional differentiation in the hippocampus." Hippocampus 8(6): 608-619.

Moser, M. B., E. I. Moser, et al. (1995). "Spatial learning with a minislab in the dorsal hippocampus." Proceedings of the National Academy of Sciences of the United States of America 92(21): 9697-9701.

Muller, J., K. P. Corodimas, et al. (1997). "Functional inactivation of the lateral and basal nuclei of the amygdala by muscimol infusion prevents fear conditioning to an explicit conditioned stimulus and to contextual stimuli." Behavioral neuroscience 111(4): 683-691.

Muller, U., N. Cristina, et al. (1994). "Behavioral and anatomical deficits in mice homozygous for a modified beta-amyloid precursor protein gene." Cell 79(5): 755-765.

Nadasdy, Z., H. Hirase, et al. (1999). "Replay and time compression of recurring spike sequences in the hippocampus." The Journal of neuroscience : the official journal of the Society for Neuroscience 19(21): 9497-9507.

Nagerl, U. V., N. Eberhorn, et al. (2004). "Bidirectional activity-dependent morphological plasticity in hippocampal neurons." Neuron 44(5): 759-767.

Nagy, P. L., J. Griesenbeck, et al. (2002). "A trithorax-group complex purified from Saccharomyces cerevisiae is required for methylation of histone H3." Proceedings of the National Academy of Sciences of the United States of America 99(1): 90-94.

Naka, F., N. Narita, et al. (2005). "Modification of AMPA receptor properties following environmental enrichment." Brain \& development 27(4): 275-278.

Nakayama, J., J. C. Rice, et al. (2001). "Role of histone H3 lysine 9 methylation in epigenetic control of heterochromatin assembly." Science 292(5514): 110-113.

Navakkode, S., S. Sajikumar, et al. (2004). "The type IV-specific phosphodiesterase inhibitor rolipram and its effect on hippocampal long-term potentiation and synaptic tagging." The Journal of neuroscience : the official journal of the Society for Neuroscience 24(35): 7740-7744.

Nelson, T. E., C. Hao, et al. (2011). "Altered hippocampal synaptic transmission in transgenic mice with astrocyte-targeted enhanced CCL2 expression." Brain, behavior, and immunity 25 Suppl 1: S106-119. 
Ng, H. H., F. Robert, et al. (2003). "Targeted recruitment of Set1 histone methylase by elongating Pol II provides a localized mark and memory of recent transcriptional activity." Molecular cell 11(3): 709719.

Nicolai, J., S. Burbassi, et al. (2010). "CXCL12 inhibits expression of the NMDA receptor's NR2B subunit through a histone deacetylase-dependent pathway contributing to neuronal survival." Cell death \& disease 1: e33.

Nithianantharajah, J. and A. J. Hannan (2006). "Enriched environments, experience-dependent plasticity and disorders of the nervous system." Nature reviews. Neuroscience 7(9): 697-709.

Noma, K., C. D. Allis, et al. (2001). "Transitions in distinct histone H3 methylation patterns at the heterochromatin domain boundaries." Science 293(5532): 1150-1155.

Oddie, S. D. and B. H. Bland (1998). "Hippocampal formation theta activity and movement selection." Neuroscience and biobehavioral reviews 22(2): 221-231.

Oliver, P. L., M. J. Finelli, et al. (2011). "Oxr1 is essential for protection against oxidative stressinduced neurodegeneration." PLoS genetics 7(10): e1002338.

Orban, P. C., D. Chui, et al. (1992). "Tissue- and site-specific DNA recombination in transgenic mice." Proceedings of the National Academy of Sciences of the United States of America 89(15): 6861-6865.

Otmakhova, N. A. and J. E. Lisman (1996). "D1/D5 dopamine receptor activation increases the magnitude of early long-term potentiation at CA1 hippocampal synapses." The Journal of neuroscience : the official journal of the Society for Neuroscience 16(23): 7478-7486.

Otto, C., Y. Kovalchuk, et al. (2001). "Impairment of mossy fiber long-term potentiation and associative learning in pituitary adenylate cyclase activating polypeptide type I receptor-deficient mice." The Journal of neuroscience : the official journal of the Society for Neuroscience 21(15): 55205527.

Otto, C., M. Martin, et al. (2001). "Altered emotional behavior in PACAP-type-I-receptor-deficient mice." Brain research. Molecular brain research 92(1-2): 78-84.

Parks, C. L., P. S. Robinson, et al. (1998). "Increased anxiety of mice lacking the serotonin1A receptor." Proceedings of the National Academy of Sciences of the United States of America 95(18): 10734-10739.

Pavlides, C. and J. Winson (1989). "Influences of hippocampal place cell firing in the awake state on the activity of these cells during subsequent sleep episodes." The Journal of neuroscience : the official journal of the Society for Neuroscience 9(8): 2907-2918. 
Peigneux, P., S. Laureys, et al. (2004). "Are spatial memories strengthened in the human hippocampus during slow wave sleep?" Neuron 44(3): 535-545.

Peigneux, P., P. Orban, et al. (2006). "Offline persistence of memory-related cerebral activity during active wakefulness." PLoS biology 4(4): e100.

Peleg, S., F. Sananbenesi, et al. (2010). "Altered histone acetylation is associated with age-dependent memory impairment in mice." Science 328(5979): 753-756.

Pentkowski, N. S., D. C. Blanchard, et al. (2006). "Effects of lesions to the dorsal and ventral hippocampus on defensive behaviors in rats." The European journal of neuroscience 23(8): 2185-2196.

Peters, A. H., J. E. Mermoud, et al. (2002). "Histone H3 lysine 9 methylation is an epigenetic imprint of facultative heterochromatin." Nature genetics 30(1): 77-80.

Peters, A. H., D. O'Carroll, et al. (2001). "Loss of the Suv39h histone methyltransferases impairs mammalian heterochromatin and genome stability." Cell 107(3): 323-337.

Petrovich, G. D., N. S. Canteras, et al. (2001). "Combinatorial amygdalar inputs to hippocampal domains and hypothalamic behavior systems." Brain research. Brain research reviews 38(1-2): 247289.

Pontzer, N. J., L. J. Chandler, et al. (1990). "Receptors, phosphoinositol hydrolysis and plasticity of nerve cells." Progress in brain research 86: 221-225.

Pothuizen, H. H., W. N. Zhang, et al. (2004). "Dissociation of function between the dorsal and the ventral hippocampus in spatial learning abilities of the rat: a within-subject, within-task comparison of reference and working spatial memory." The European journal of neuroscience 19(3): 705-712.

Price, J. L. (2007). "Definition of the orbital cortex in relation to specific connections with limbic and visceral structures and other cortical regions." Annals of the New York Academy of Sciences 1121: 54-71.

Qian, Z., M. Gilbert, et al. (1994). "Temporal and spatial regulation of the expression of BAD2, a MAP kinase phosphatase, during seizure, kindling, and long-term potentiation." Learning \& memory 1(3): 180-188.

Qian, Z., M. E. Gilbert, et al. (1993). "Tissue-plasminogen activator is induced as an immediate-early gene during seizure, kindling and long-term potentiation." Nature 361(6411): 453-457.

Quinn, M. R. and C. L. Harris (1995). "Lead inhibits Ca(2+)-stimulated nitric oxide synthase activity from rat cerebellum." Neuroscience letters 196(1-2): 65-68. 
Ramanan, N., Y. Shen, et al. (2005). "SRF mediates activity-induced gene expression and synaptic plasticity but not neuronal viability." Nature neuroscience 8(6): 759-767.

Rampon, C., Y. P. Tang, et al. (2000). "Enrichment induces structural changes and recovery from nonspatial memory deficits in CA1 NMDAR1-knockout mice." Nature neuroscience 3(3): 238-244.

Reissner, K. J., J. L. Shobe, et al. (2006). "Molecular nodes in memory processing: insights from Aplysia." Cellular and molecular life sciences : CMLS 63(9): 963-974.

Restivo, L., G. Vetere, et al. (2009). "The formation of recent and remote memory is associated with time-dependent formation of dendritic spines in the hippocampus and anterior cingulate cortex." The Journal of neuroscience : the official journal of the Society for Neuroscience 29(25): 8206-8214.

Reul, J. M., S. A. Hesketh, et al. (2009). "Epigenetic mechanisms in the dentate gyrus act as a molecular switch in hippocampus-associated memory formation." Epigenetics : official journal of the DNA Methylation Society 4(7): 434-439.

Reymann, K. G., U. Frey, et al. (1988). "Polymyxin B, an inhibitor of protein kinase C, prevents the maintenance of synaptic long-term potentiation in hippocampal CA1 neurons." Brain research 440(2): 305-314.

Reymann, K. G., R. Malisch, et al. (1985). "The duration of long-term potentiation in the CA1 region of the hippocampal slice preparation." Brain research bulletin 15(3): 249-255.

Ribeiro, R. L., R. Andreatini, et al. (1999). "The "anxiety state" and its relation with rat models of memory and habituation." Neurobiology of learning and memory 72(2): 78-94.

Ribeiro, S., D. Gervasoni, et al. (2004). "Long-lasting novelty-induced neuronal reverberation during slow-wave sleep in multiple forebrain areas." PLoS biology 2(1): E24.

Ribeiro, S., C. V. Mello, et al. (2002). "Induction of hippocampal long-term potentiation during waking leads to increased extrahippocampal zif-268 expression during ensuing rapid-eye-movement sleep." The Journal of neuroscience : the official journal of the Society for Neuroscience 22(24): 10914-10923.

Risold, P. Y. and L. W. Swanson (1996). "Structural evidence for functional domains in the rat hippocampus." Science 272(5267): 1484-1486.

Risold, P. Y., R. H. Thompson, et al. (1997). "The structural organization of connections between hypothalamus and cerebral cortex." Brain Research Reviews 24(2-3): 197-254.

Roberson, E. D., J. D. English, et al. (1999). "The mitogen-activated protein kinase cascade couples PKA and PKC to cAMP response element binding protein phosphorylation in area CA1 of 
hippocampus." The Journal of neuroscience : the official journal of the Society for Neuroscience 19(11): 4337-4348.

Roguev, A., D. Schaft, et al. (2001). "The Saccharomyces cerevisiae Set1 complex includes an Ash2 homologue and methylates histone 3 lysine 4." The EMBO journal 20(24): 7137-7148.

Rosenzweig, M. R. and E. L. Bennett (1996). "Psychobiology of plasticity: effects of training and experience on brain and behavior." Behavioural brain research 78(1): 57-65.

Ross, S. A., J. Y. Wong, et al. (2000). "Phenotypic characterization of an alpha 4 neuronal nicotinic acetylcholine receptor subunit knock-out mouse." The Journal of neuroscience : the official journal of the Society for Neuroscience 20(17): 6431-6441.

Rossi-Arnaud, C., S. Fagioli, et al. (1991). "Spatial learning in two inbred strains of mice: genotypedependent effect of amygdaloid and hippocampal lesions." Behavioural brain research 45(1): 9-16.

Roy, V., C. Belzung, et al. (2001). "Environmental enrichment in BALB/c mice: effects in classical tests of anxiety and exposure to a predatory odor." Physiology \& behavior 74(3): 313-320.

Sadler, T. R., P. T. Nguyen, et al. (2011). "Antenatal maternal stress alters functional brain responses in adult offspring during conditioned fear." Brain research 1385: 163-174.

Salin, P. A., R. C. Malenka, et al. (1996). "Cyclic AMP mediates a presynaptic form of LTP at cerebellar parallel fiber synapses." Neuron 16(4): 797-803.

Sanfeliu, C., A. Hunt, et al. (1990). "Exposure to N-methyl-D-aspartate increases release of arachidonic acid in primary cultures of rat hippocampal neurons and not in astrocytes." Brain research 526(2): 241-248.

Santos-Rosa, H., R. Schneider, et al. (2002). "Active genes are tri-methylated at K4 of histone H3." Nature 419(6905): 407-411.

Sarnyai, Z., E. L. Sibille, et al. (2000). "Impaired hippocampal-dependent learning and functional abnormalities in the hippocampus in mice lacking serotonin(1A) receptors." Proceedings of the National Academy of Sciences of the United States of America 97(26): 14731-14736.

Sartori, S. B., M. Hauschild, et al. (2011). "Enhanced fear expression in a psychopathological mouse model of trait anxiety: pharmacological interventions." PLoS ONE 6(2): e16849.

Saywell, N. and D. Taylor (2008). "The role of the cerebellum in procedural learning--are there implications for physiotherapists' clinical practice?" Physiotherapy theory and practice 24(5): 321-328.

Schaefer, A., S. C. Sampath, et al. (2009). "Control of cognition and adaptive behavior by the GLP/G9a epigenetic suppressor complex." Neuron 64(5): 678-691. 
Scharf, A. N. and A. Imhof (2011). "Every methyl counts--epigenetic calculus." FEBS letters 585(13): 2001-2007.

Schneider, J., A. Wood, et al. (2005). "Molecular regulation of histone H3 trimethylation by COMPASS and the regulation of gene expression." Molecular cell 19(6): 849-856.

Schuettengruber, B., M. Ganapathi, et al. (2009). "Functional anatomy of polycomb and trithorax chromatin landscapes in Drosophila embryos." PLoS biology 7(1): e13.

Schwartz, Y. B., T. G. Kahn, et al. (2006). "Genome-wide analysis of Polycomb targets in Drosophila melanogaster." Nature genetics 38(6): 700-705.

Schwegler, H. and W. E. Crusio (1995). "Correlations between radial-maze learning and structural variations of septum and hippocampus in rodents." Behavioural brain research 67(1): 29-41.

Schwindel, C. D. and B. L. McNaughton (2011). "Hippocampal-cortical interactions and the dynamics of memory trace reactivation." Progress in brain research 193: 163-177.

Scoville, W. B. and B. Milner (1957). "Loss of recent memory after bilateral hippocampal lesions." Journal of neurology, neurosurgery, and psychiatry 20(1): 11-21.

Segal, M. (2005). "Dendritic spines and long-term plasticity." Nature reviews. Neuroscience 6(4): 277284.

Sewards, T. V. and M. A. Sewards (2002). "The medial pain system: Neural representations of the motivational aspect of pain." Brain Research Bulletin 59(3): 163-180.

Shi, Y., F. Lan, et al. (2004). "Histone demethylation mediated by the nuclear amine oxidase homolog LSD1." Cell 119(7): 941-953.

Shi, Y. and J. R. Whetstine (2007). "Dynamic regulation of histone lysine methylation by demethylases." Molecular cell 25(1): 1-14.

Shibuki, K. and D. Okada (1992). "Cerebellar long-term potentiation under suppressed postsynaptic Ca2+ activity." Neuroreport 3(3): 231-234.

Shilatifard, A. (2006). "Chromatin modifications by methylation and ubiquitination: implications in the regulation of gene expression." Annual review of biochemistry 75: 243-269.

Shilatifard, A. (2008). "Molecular implementation and physiological roles for histone H3 lysine 4 (H3K4) methylation." Current opinion in cell biology 20(3): 341-348.

Shimizu, E., Y. P. Tang, et al. (2000). "NMDA receptor-dependent synaptic reinforcement as a crucial process for memory consolidation." Science 290(5494): 1170-1174. 
Sibille, E., C. Pavlides, et al. (2000). "Genetic inactivation of the Serotonin(1A) receptor in mice results in downregulation of major $\mathrm{GABA}(\mathrm{A})$ receptor alpha subunits, reduction of GABA(A) receptor binding, and benzodiazepine-resistant anxiety." The Journal of neuroscience : the official journal of the Society for Neuroscience 20(8): 2758-2765.

Sims, R. J., 3rd, K. Nishioka, et al. (2003). "Histone lysine methylation: a signature for chromatin function." Trends in genetics : TIG 19(11): 629-639.

Sims, R. J., 3rd and D. Reinberg (2006). "Histone H3 Lys 4 methylation: caught in a bind?" Genes \& development 20(20): 2779-2786.

Sindreu, C. B., Z. S. Scheiner, et al. (2007). "Ca2+ -stimulated adenylyl cyclases regulate ERKdependent activation of MSK1 during fear conditioning." Neuron 53(1): 79-89.

Smyth, G. K. (2004). "Linear models and empirical bayes methods for assessing differential expression in microarray experiments." Statistical applications in genetics and molecular biology $\mathbf{3}$ : Article3.

Steward, M. M., J. S. Lee, et al. (2006). "Molecular regulation of H3K4 trimethylation by ASH2L, a shared subunit of MLL complexes." Nature structural \& molecular biology 13(9): 852-854.

Strahl, B. D. and C. D. Allis (2000). "The language of covalent histone modifications." Nature 403(6765): 41-45.

Sudhof, T. C., F. Lottspeich, et al. (1987). "A synaptic vesicle protein with a novel cytoplasmic domain and four transmembrane regions." Science 238(4830): 1142-1144.

Sun, H., C. H. Charles, et al. (1993). "MKP-1 (3CH134), an immediate early gene product, is a dual specificity phosphatase that dephosphorylates MAP kinase in vivo." Cell 75(3): 487-493.

Sun, M., D. Dahl, et al. (2001). "Heterosynaptic transformation of GABAergic gating in the hippocampus and effects of carbonic anhydrase inhibition." The Journal of pharmacology and experimental therapeutics 296(3): 811-817.

Sun, M. K. and D. L. Alkon (2001). "Pharmacological enhancement of synaptic efficacy, spatial learning, and memory through carbonic anhydrase activation in rats." The Journal of pharmacology and experimental therapeutics 297(3): 961-967.

Sun, M. K., T. J. Nelson, et al. (2000). "Functional switching of GABAergic synapses by ryanodine receptor activation." Proceedings of the National Academy of Sciences of the United States of America 97(22): 12300-12305. 
Swanson, L. W. and W. M. Cowan (1977). "An autoradiographic study of the organization of the efferent connections of the hippocampal formation in the rat." The Journal of comparative neurology 172(1): 49-84.

Sweatt, J. D. (2001). "Memory mechanisms: the yin and yang of protein phosphorylation." Current biology: CB 11(10): R391-394.

Sweatt, J. D. (2004). "Mitogen-activated protein kinases in synaptic plasticity and memory." Current Opinion in Neurobiology 14(3): 311-317.

Tachibana, M., K. Sugimoto, et al. (2001). "Set domain-containing protein, G9a, is a novel lysinepreferring mammalian histone methyltransferase with hyperactivity and specific selectivity to lysines 9 and 27 of histone H3." The Journal of biological chemistry 276(27): 25309-25317.

Tang, Y. P., H. Wang, et al. (2001). "Differential effects of enrichment on learning and memory function in NR2B transgenic mice." Neuropharmacology 41(6): 779-790.

Taubenfeld, S. M., K. A. Wiig, et al. (1999). "A molecular correlate of memory and amnesia in the hippocampus." Nature neuroscience 2(4): 309-310.

Teixeira, C. M., S. R. Pomedli, et al. (2006). "Involvement of the anterior cingulate cortex in the expression of remote spatial memory." The Journal of neuroscience : the official journal of the Society for Neuroscience 26(29): 7555-7564.

Tenney, K. and A. Shilatifard (2005). "A COMPASS in the voyage of defining the role of trithorax/MLL-containing complexes: linking leukemogensis to covalent modifications of chromatin." Journal of cellular biochemistry 95(3): 429-436.

Terranova, R., H. Agherbi, et al. (2006). "Histone and DNA methylation defects at Hox genes in mice expressing a SET domain-truncated form of Mll." Proceedings of the National Academy of Sciences of the United States of America 103(17): 6629-6634.

Thomas, G. M. and R. L. Huganir (2004). "MAPK cascade signalling and synaptic plasticity." Nature reviews. Neuroscience 5(3): 173-183.

Thomas, M., A. Gessner, et al. (2005). "Targeting MLL-AF4 with short interfering RNAs inhibits clonogenicity and engraftment of $\mathrm{t}(4 ; 11)$-positive human leukemic cells." Blood 106(10): 3559-3566.

Thompson, C. L., S. D. Pathak, et al. (2008). "Genomic anatomy of the hippocampus." Neuron 60(6): 1010-1021.

Tischmeyer, W. and R. Grimm (1999). "Activation of immediate early genes and memory formation." Cellular and molecular life sciences : CMLS 55(4): 564-574. 
Tsien, J. Z., D. F. Chen, et al. (1996). "Subregion- and cell type-restricted gene knockout in mouse brain." Cell 87(7): 1317-1326.

Turner, A. M. and W. T. Greenough (1985). "Differential rearing effects on rat visual cortex synapses. I. Synaptic and neuronal density and synapses per neuron." Brain research 329(1-2): 195-203.

Ullman, M. T. (2001). "A neurocognitive perspective on language: the declarative/procedural model." Nature reviews. Neuroscience 2(10): 717-726.

Ullman, M. T. (2004). "Contributions of memory circuits to language: the declarative/procedural model." Cognition 92(1-2): 231-270.

Ullman, M. T. and E. I. Pierpont (2005). "Specific language impairment is not specific to language: the procedural deficit hypothesis." Cortex; a journal devoted to the study of the nervous system and behavior 41(3): 399-433.

Valor, L. M., M. M. Pulopulos, et al. (2011). "Ablation of CBP in forebrain principal neurons causes modest memory and transcriptional defects and a dramatic reduction of histone acetylation but does not affect cell viability." The Journal of neuroscience : the official journal of the Society for Neuroscience 31(5): 1652-1663.

van Praag, H., G. Kempermann, et al. (2000). "Neural consequences of environmental enrichment." Nature reviews. Neuroscience 1(3): 191-198.

Vaquero, A., A. Loyola, et al. (2003). "The constantly changing face of chromatin." Science of aging knowledge environment : SAGE KE 2003(14): RE4.

Vecsey, C. G., J. D. Hawk, et al. (2007). "Histone deacetylase inhibitors enhance memory and synaptic plasticity via CREB:CBP-dependent transcriptional activation." The Journal of neuroscience : the official journal of the Society for Neuroscience 27(23): 6128-6140.

Venero, C., A. Guadano-Ferraz, et al. (2005). "Anxiety, memory impairment, and locomotor dysfunction caused by a mutant thyroid hormone receptor alpha1 can be ameliorated by T3 treatment." Genes \& development 19(18): 2152-2163.

Vertes, R. P. (2006). "Interactions among the medial prefrontal cortex, hippocampus and midline thalamus in emotional and cognitive processing in the rat." Neuroscience 142(1): 1-20.

Vlkolinsky, R., G. R. Siggins, et al. (2004). "Acute exposure to CXC chemokine ligand 10, but not its chronic astroglial production, alters synaptic plasticity in mouse hippocampal slices." 吕urnal of neuroimmunology 150(1-2): 37-47. 
Volkert, M. R., N. A. Elliott, et al. (2000). "Functional genomics reveals a family of eukaryotic oxidation protection genes." Proceedings of the National Academy of Sciences of the United States of America 97(26): 14530-14535.

Volpe, T. A., C. Kidner, et al. (2002). "Regulation of heterochromatic silencing and histone H3 lysine9 methylation by RNAi." Science 297(5588): 1833-1837.

Wall, P. M. and C. Messier (2000). "Concurrent modulation of anxiety and memory." Behavioural brain research 109(2): 229-241.

Wang, G. G., L. Cai, et al. (2007). "NUP98-NSD1 links H3K36 methylation to Hox-A gene activation and leukaemogenesis." Nature cell biology 9(7): 804-812.

Wang, Z., C. Zang, et al. (2009). "Genome-wide mapping of HATs and HDACs reveals distinct functions in active and inactive genes." Cell 138(5): 1019-1031.

Wheal, H. V., B. Lancaster, et al. (1983). "Long-term potentiation in Schaffer collateral and commissural systems of the hippocampus: in vitro study in rats pretreated with kainic acid." Brain research 272(2): 247-253.

Wiedenmann, B. and W. W. Franke (1985). "Identification and localization of synaptophysin, an integral membrane glycoprotein of Mr 38,000 characteristic of presynaptic vesicles." Cell 41(3): 10171028.

Wilson, M. A. and B. L. McNaughton (1994). "Reactivation of hippocampal ensemble memories during sleep." Science 265(5172): 676-679.

Wolf, M. J., Y. Izumi, et al. (1995). "Long-term potentiation requires activation of calciumindependent phospholipase A2." FEBS letters 377(3): 358-362.

Wood, A., A. Shukla, et al. (2007). "Ctk complex-mediated regulation of histone methylation by COMPASS." Molecular and cellular biology 27(2): 709-720.

Wood, M. A., M. A. Attner, et al. (2006). "A transcription factor-binding domain of the coactivator CBP is essential for long-term memory and the expression of specific target genes." Learning \& memory 13(5): 609-617.

Wysocka, J., T. Swigut, et al. (2005). "WDR5 associates with histone H3 methylated at K4 and is essential for H3 K4 methylation and vertebrate development." Cell 121(6): 859-872.

Yagi, H., K. Deguchi, et al. (1998). "Growth disturbance in fetal liver hematopoiesis of Mll-mutant mice." Blood 92(1): 108-117. 
Yang, F., X. He, et al. (2001). "PI-3 kinase and IP3 are both necessary and sufficient to mediate NT3induced synaptic potentiation." Nature neuroscience 4(1): 19-28.

Yang, Y. J., P. F. Wu, et al. (2010). "Reversal of aging-associated hippocampal synaptic plasticity deficits by reductants via regulation of thiol redox and NMDA receptor function." Aging cell 9(5): 709-721.

Yeh, S. H., C. H. Lin, et al. (2002). "A requirement of nuclear factor-kappaB activation in fearpotentiated startle." The Journal of biological chemistry 277(48): 46720-46729.

Yu, B. D., J. L. Hess, et al. (1995). "Altered Hox expression and segmental identity in Mll-mutant mice." Nature 378(6556): 505-508.

Zhang, J., M. I. Miller, et al. (2005). "Mapping postnatal mouse brain development with diffusion tensor microimaging." NeuroImage 26(4): 1042-1051.

Zorner, B., D. P. Wolfer, et al. (2003). "Forebrain-specific trkB-receptor knockout mice: behaviorally more hyperactive than "depressive"." Biological psychiatry 54(10): 972-982. 


\section{Appendix 1}

Table 1: List of differentially regulated genes in dorsal dentate gyrus of F/F CKII mice (the genes in red were confirmed by qRT-PCR)

\begin{tabular}{|c|c|c|c|}
\hline Symbol & Full Name & $\begin{array}{l}\text { log (fold } \\
\text { change) }\end{array}$ & $\begin{array}{l}\text { Adjusted } p \text { - } \\
\text { value }\end{array}$ \\
\hline Vsig8 & $\begin{array}{l}\mathrm{V} \text {-set and immunoglobulin } \\
\text { domain containing } 8\end{array}$ & $-2,88$ & $5,42 \mathrm{E}-07$ \\
\hline Hfm1 & $\begin{array}{l}\text { HFM1, ATP-dependent DNA } \\
\text { helicase homolog (S. } \\
\text { cerevisiae) }\end{array}$ & $-2,62$ & $1,32 \mathrm{E}-07$ \\
\hline Efhc2 & $\begin{array}{c}\text { EF-hand domain (C-terminal) } \\
\text { containing } 2\end{array}$ & $-2,57$ & $9,75 \mathrm{E}-05$ \\
\hline Nkapl & NFKB activating protein-like & $-2,52$ & $1,04 \mathrm{E}-05$ \\
\hline Dazl & deleted in azoospermia-like & $-2,50$ & $1,98 \mathrm{E}-06$ \\
\hline Igsf1 & $\begin{array}{l}\text { immunoglobulin superfamily, } \\
\text { member } 1\end{array}$ & $-2,33$ & $1,89 \mathrm{E}-06$ \\
\hline Lor & loricrin & $-2,33$ & $5,94 \mathrm{E}-11$ \\
\hline $\mathrm{Hdx}$ & highly divergent homeobox & $-2,17$ & $8,19 \mathrm{E}-08$ \\
\hline $\mathrm{Fbx} 17$ & $\begin{array}{l}\text { F-box and leucine-rich repeat } \\
\text { protein } 7\end{array}$ & $-2,13$ & $1,02 \mathrm{E}-05$ \\
\hline Wtip & WT1-interacting protein & $-2,05$ & $1,21 \mathrm{E}-07$ \\
\hline Prss16 & protease, serine, 16 (thymus) & $-2,02$ & $2,40 \mathrm{E}-04$ \\
\hline Zfp459 & zinc finger protein 459 & $-1,96$ & $6,67 \mathrm{E}-06$ \\
\hline Wdr17 & WD repeat domain 17 & $-1,92$ & $1,63 \mathrm{E}-08$ \\
\hline Rab38 & $\begin{array}{l}\text { RAB38, member of RAS } \\
\text { oncogene family }\end{array}$ & $-1,90$ & $1,31 \mathrm{E}-04$ \\
\hline Tmem22 & transmembrane protein 22 & $-1,89$ & $3,44 \mathrm{E}-07$ \\
\hline Lrrn4 & leucine rich repeat neuronal 4 & $-1,89$ & $4,97 \mathrm{E}-06$ \\
\hline Prr15 & proline rich 15 & $-1,89$ & $3,49 \mathrm{E}-06$ \\
\hline Ap1s3 & $\begin{array}{l}\text { adaptor-related protein } \\
\text { complex AP-1, sigma } 3\end{array}$ & $-1,84$ & $5,45 \mathrm{E}-07$ \\
\hline Gprc5c & $\begin{array}{l}\text { G protein-coupled receptor, } \\
\text { family } \mathrm{C} \text {, group } 5 \text {, member } \mathrm{C}\end{array}$ & $-1,81$ & $8,53 \mathrm{E}-05$ \\
\hline Nmnat3 & $\begin{array}{c}\text { nicotinamide nucleotide } \\
\text { adenylyltransferase } 3\end{array}$ & $-1,77$ & $6,13 \mathrm{E}-07$ \\
\hline Gm9934 & predicted gene 9934 & $-1,74$ & $5,01 \mathrm{E}-05$ \\
\hline$A \cot 4$ & acyl-CoA thioesterase 4 & $-1,74$ & $4,45 \mathrm{E}-05$ \\
\hline Tceall & $\begin{array}{l}\text { transcription elongation factor } \\
\text { A (SII)-like } 1\end{array}$ & $-1,72$ & $1,74 \mathrm{E}-07$ \\
\hline Cd55 & CD55 antigen & $-1,71$ & $2,54 \mathrm{E}-04$ \\
\hline BB557941 & expressed sequence BB557941 & $-1,70$ & $1,79 \mathrm{E}-06$ \\
\hline Rimklb & $\begin{array}{l}\text { ribosomal modification protein } \\
\text { rimK-like family member B }\end{array}$ & $-1,69$ & $1,62 \mathrm{E}-06$ \\
\hline Gprc5c & $\begin{array}{l}\text { G protein-coupled receptor, } \\
\text { family } \mathrm{C} \text {, group } 5 \text {, member } \mathrm{C}\end{array}$ & $-1,69$ & $1,29 \mathrm{E}-04$ \\
\hline Dusp2 & dual specificity phosphatase 2 & $-1,67$ & $8,38 \mathrm{E}-07$ \\
\hline Tcf15 & transcription factor 15 & $-1,66$ & $1,16 \mathrm{E}-06$ \\
\hline
\end{tabular}




\begin{tabular}{|c|c|c|c|}
\hline Tmem159 & transmembrane protein 159 & $-1,66$ & $1,72 \mathrm{E}-05$ \\
\hline Artn & artemin & $-1,61$ & $5,81 \mathrm{E}-05$ \\
\hline Naaladl2 & $\begin{array}{l}\mathrm{N} \text {-acetylated alpha-linked } \\
\text { acidic dipeptidase-like } 2\end{array}$ & $-1,55$ & $1,68 \mathrm{E}-07$ \\
\hline Ltk & leukocyte tyrosine kinase & $-1,55$ & $3,62 \mathrm{E}-05$ \\
\hline Zfp184 & $\begin{array}{l}\text { zinc finger protein } 184 \\
\text { (Kruppel-like) }\end{array}$ & $-1,55$ & $2,88 \mathrm{E}-05$ \\
\hline Zfp647 & zinc finger protein 647 & $-1,53$ & $3,82 \mathrm{E}-08$ \\
\hline Gm2960 & predicted gene 2960 & $-1,53$ & $4,08 \mathrm{E}-05$ \\
\hline Mfsd9 & $\begin{array}{l}\text { major facilitator superfamily } \\
\text { domain containing } 9\end{array}$ & $-1,52$ & $2,48 \mathrm{E}-07$ \\
\hline Zfp51 & zinc finger protein 51 & $-1,48$ & $3,01 \mathrm{E}-07$ \\
\hline Six 4 & $\begin{array}{c}\text { sine oculis-related homeobox } 4 \\
\text { homolog (Drosophila) }\end{array}$ & $-1,46$ & $7,90 \mathrm{E}-05$ \\
\hline Rad51ap2 & RAD51 associated protein 2 & $-1,46$ & $2,69 \mathrm{E}-05$ \\
\hline Mir17hg & $\begin{array}{l}\text { MIR17 host gene } 1 \text { (non- } \\
\text { protein coding) }\end{array}$ & $-1,45$ & $1,54 \mathrm{E}-04$ \\
\hline Ccdc160 & $\begin{array}{l}\text { coiled-coil domain containing } \\
160 \\
\end{array}$ & $-1,45$ & $3,64 \mathrm{E}-04$ \\
\hline Tusc1 & tumor suppressor candidate 1 & $-1,44$ & $1,47 \mathrm{E}-06$ \\
\hline Nipsnap3b & $\begin{array}{c}\text { nipsnap homolog 3B (C. } \\
\text { elegans) }\end{array}$ & $-1,43$ & $1,14 \mathrm{E}-05$ \\
\hline Haus4 & $\begin{array}{l}\text { HAUS augmin-like complex, } \\
\text { subunit } 4\end{array}$ & $-1,42$ & $1,47 \mathrm{E}-06$ \\
\hline Nipsnap3b & $\begin{array}{c}\text { nipsnap homolog 3B (C. } \\
\text { elegans) }\end{array}$ & $-1,38$ & $6,12 \mathrm{E}-07$ \\
\hline Gm11818 & predicted gene 11818 & $-1,36$ & $9,85 \mathrm{E}-05$ \\
\hline Zfp760 & zinc finger protein 760 & $-1,35$ & $3,55 \mathrm{E}-05$ \\
\hline Wdsub1 & $\begin{array}{l}\text { WD repeat, SAM and U-box } \\
\text { domain containing } 1\end{array}$ & $-1,35$ & $2,46 \mathrm{E}-06$ \\
\hline Adcy5 & adenylate cyclase 5 & $-1,34$ & 9,63E-06 \\
\hline Fam117a & $\begin{array}{l}\text { family with sequence similarity } \\
117, \text { memberA }\end{array}$ & $-1,32$ & $6,59 \mathrm{E}-06$ \\
\hline Ccdc91 & $\begin{array}{l}\text { coiled-coil domain containing } \\
91\end{array}$ & $-1,31$ & $8,47 \mathrm{E}-09$ \\
\hline Foxq1 & forkhead box Q1 & $-1,31$ & $2,02 \mathrm{E}-05$ \\
\hline Metrnl & $\begin{array}{c}\text { meteorin, glial cell } \\
\text { differentiation regulator-like }\end{array}$ & $-1,29$ & $4,01 \mathrm{E}-07$ \\
\hline Adcy5 & adenylate cyclase 5 & $-1,27$ & 9,03E-07 \\
\hline Zfp641 & zinc finger protein 641 & $-1,27$ & $8,36 \mathrm{E}-06$ \\
\hline Ccdc111 & $\begin{array}{l}\text { coiled-coil domain containing } \\
111\end{array}$ & $-1,26$ & $1,91 \mathrm{E}-04$ \\
\hline Ptgr1 & prostaglandin reductase 1 & $-1,25$ & $1,89 \mathrm{E}-04$ \\
\hline Neil2 & nei like 2 (E. coli) & $-1,25$ & $4,00 \mathrm{E}-04$ \\
\hline Car10 & carbonic anhydrase 10 & $-1,24$ & $4,18 \mathrm{E}-05$ \\
\hline Ikzf2 & IKAROS family zinc finger 2 & $-1,24$ & $2,23 \mathrm{E}-04$ \\
\hline Prkra & $\begin{array}{c}\text { protein kinase, interferon } \\
\text { inducible double stranded RNA } \\
\text { dependent activator }\end{array}$ & $-1,22$ & $7,15 \mathrm{E}-07$ \\
\hline Immp21 & $\begin{array}{l}\text { IMP2 inner mitochondrial } \\
\text { membrane peptidase-like }(\mathrm{S} \text {. }\end{array}$ & $-1,22$ & $2,31 \mathrm{E}-07$ \\
\hline
\end{tabular}




\begin{tabular}{|c|c|c|c|}
\hline & cerevisiae) & & \\
\hline Gabrg3 & $\begin{array}{l}\text { gamma-aminobutyric acid } \\
\text { (GABA) A receptor, subunit } \\
\text { gamma 3 }\end{array}$ & $-1,22$ & $3,64 \mathrm{E}-04$ \\
\hline Ikzf2 & IKAROS family zinc finger 2 & $-1,21$ & $2,94 \mathrm{E}-05$ \\
\hline Zfp429 & zinc finger protein 429 & $-1,20$ & $1,71 \mathrm{E}-05$ \\
\hline Xkrx & $\begin{array}{l}\text { X Kell blood group precursor } \\
\text { related X linked }\end{array}$ & $-1,17$ & $3,53 \mathrm{E}-05$ \\
\hline Adcy5 & adenylate cyclase 5 & $-1,16$ & $2,80 \mathrm{E}-05$ \\
\hline Glyctk & glycerate kinase & $-1,14$ & $2,41 \mathrm{E}-04$ \\
\hline Marveld1 & $\begin{array}{c}\text { MARVEL (membrane- } \\
\text { associating) domain containing } \\
1\end{array}$ & $-1,13$ & $2,11 \mathrm{E}-04$ \\
\hline Nt5dc1 & $\begin{array}{c}\text { 5'-nucleotidase domain } \\
\text { containing } 1\end{array}$ & $-1,13$ & $5,42 \mathrm{E}-05$ \\
\hline Syde2 & $\begin{array}{c}\text { synapse defective } 1, \text { Rho } \\
\text { GTPase, homolog } 2 \text { (C. } \\
\text { elegans) }\end{array}$ & $-1,13$ & $2,31 \mathrm{E}-05$ \\
\hline Ppic & peptidylprolyl isomerase $\mathrm{C}$ & $-1,12$ & 1,79E-04 \\
\hline Pole4 & $\begin{array}{l}\text { polymerase (DNA-directed) } \\
\text { epsilon } 4 \text { (p12 subunit) }\end{array}$ & $-1,12$ & $1,42 \mathrm{E}-05$ \\
\hline Car4 & carbonic anhydrase 4 & $-1,11$ & $1,30 \mathrm{E}-04$ \\
\hline Tox3 & $\begin{array}{l}\text { TOX high mobility group box } \\
\text { family member } 3\end{array}$ & $-1,11$ & $3,61 \mathrm{E}-04$ \\
\hline Larp1b & $\begin{array}{l}\text { La ribonucleoprotein domain } \\
\text { family, member 1B }\end{array}$ & $-1,10$ & $1,27 \mathrm{E}-05$ \\
\hline Myom3 & myomesin family, member 3 & $-1,10$ & $1,56 \mathrm{E}-05$ \\
\hline Sord & sorbitol dehydrogenase & $-1,08$ & 4,44E-07 \\
\hline Oxr1 & oxidation resistance 1 & $-1,08$ & 5,91E-07 \\
\hline Fah & fumarylacetoacetate hydrolase & $-1,07$ & $1,11 \mathrm{E}-06$ \\
\hline Ckap4 & $\begin{array}{c}\text { cytoskeleton-associated protein } \\
4\end{array}$ & $-1,07$ & $6,12 \mathrm{E}-07$ \\
\hline Paox & $\begin{array}{c}\text { polyamine oxidase (exo-N4- } \\
\text { amino) }\end{array}$ & $-1,07$ & $2,84 \mathrm{E}-07$ \\
\hline Zfp612 & zinc finger protein 612 & $-1,07$ & $1,12 \mathrm{E}-05$ \\
\hline Fbxl21 & $\begin{array}{l}\text { F-box and leucine-rich repeat } \\
\text { protein } 21\end{array}$ & $-1,07$ & $4,96 \mathrm{E}-05$ \\
\hline Stxbp2 & syntaxin binding protein 2 & $-1,06$ & $3,16 \mathrm{E}-04$ \\
\hline Btg3 & B-cell translocation gene 3 & $-1,06$ & $4,53 \mathrm{E}-05$ \\
\hline Kdelc2 & $\begin{array}{l}\text { KDEL (Lys-Asp-Glu-Leu) } \\
\text { containing } 2 \\
\end{array}$ & $-1,05$ & $3,71 \mathrm{E}-06$ \\
\hline Rimbp3 & RIMS binding protein 3 & $-1,02$ & $1,60 \mathrm{E}-04$ \\
\hline Paox & $\begin{array}{l}\text { polyamine oxidase (exo-N4- } \\
\text { amino) }\end{array}$ & $-1,02$ & $1,11 \mathrm{E}-06$ \\
\hline Gm347 & predicted gene 347 & $-1,00$ & $6,61 \mathrm{E}-05$ \\
\hline Irf1 & interferon regulatory factor 1 & $-0,99$ & 1,29E-06 \\
\hline B3gnt6 & $\begin{array}{l}\text { UDP-GlcNAc:betaGal beta- } \\
1,3-\mathrm{N}- \\
\text { acetylglucosaminyltransferase } \\
6 \text { (core } 3 \text { synthase) }\end{array}$ & $-0,98$ & $3,05 \mathrm{E}-04$ \\
\hline Gpx7 & glutathione peroxidase 7 & $-0,97$ & $2,62 \mathrm{E}-05$ \\
\hline
\end{tabular}




\begin{tabular}{|c|c|c|c|}
\hline Stbd1 & starch binding domain 1 & $-0,97$ & $5,00 \mathrm{E}-05$ \\
\hline Rabepk & $\begin{array}{l}\text { Rab9 effector protein with } \\
\text { kelch motifs }\end{array}$ & $-0,97$ & $1,13 \mathrm{E}-05$ \\
\hline Bag2 & BCL2-associated athanogene 2 & $-0,97$ & $5,25 \mathrm{E}-07$ \\
\hline Ptgr2 & prostaglandin reductase 2 & $-0,97$ & $6,91 \mathrm{E}-06$ \\
\hline Gm11110 & predicted gene 11110 & $-0,96$ & $1,70 \mathrm{E}-04$ \\
\hline Parp11 & $\begin{array}{l}\text { poly (ADP-ribose) polymerase } \\
\text { family, member } 11\end{array}$ & $-0,94$ & $8,40 \mathrm{E}-07$ \\
\hline Gk5 & glycerol kinase 5 (putative) & $-0,92$ & $6,62 \mathrm{E}-05$ \\
\hline Pole & $\begin{array}{l}\text { polymerase (DNA directed), } \\
\text { epsilon }\end{array}$ & $-0,92$ & $7,68 \mathrm{E}-05$ \\
\hline Chst7 & $\begin{array}{l}\text { carbohydrate }(\mathrm{N}- \\
\text { acetylglucosamino) } \\
\text { sulfotransferase } 7\end{array}$ & $-0,92$ & $1,05 \mathrm{E}-04$ \\
\hline Echdc2 & $\begin{array}{l}\text { enoyl Coenzyme A hydratase } \\
\text { domain containing } 2\end{array}$ & $-0,92$ & $6,50 \mathrm{E}-06$ \\
\hline Bves & $\begin{array}{l}\text { blood vessel epicardial } \\
\text { substance }\end{array}$ & $-0,91$ & $2,06 \mathrm{E}-05$ \\
\hline Sypl2 & synaptophysin-like 2 & $-0,90$ & $7,01 \mathrm{E}-05$ \\
\hline Neu3 & neuraminidase 3 & $-0,90$ & 7,09E-06 \\
\hline Acot1 & acyl-CoA thioesterase 1 & $-0,89$ & $1,02 \mathrm{E}-05$ \\
\hline Jag2 & jagged 2 & $-0,89$ & $1,84 \mathrm{E}-04$ \\
\hline Cntln & centlein, centrosomal protein & $-0,89$ & $1,70 \mathrm{E}-05$ \\
\hline Rab42-ps & $\begin{array}{c}\text { RAB42, member RAS } \\
\text { oncogene family, pseudogene }\end{array}$ & $-0,89$ & $2,84 \mathrm{E}-04$ \\
\hline Nudt14 & $\begin{array}{l}\text { nudix (nucleoside diphosphate } \\
\text { linked moiety X)-type motif } 14\end{array}$ & $-0,87$ & $3,32 \mathrm{E}-05$ \\
\hline Lck & $\begin{array}{c}\text { lymphocyte protein tyrosine } \\
\text { kinase }\end{array}$ & $-0,84$ & $2,01 \mathrm{E}-04$ \\
\hline Rell1 & RELT-like 1 & $-0,84$ & $7,22 \mathrm{E}-06$ \\
\hline Slc1a6 & $\begin{array}{l}\text { solute carrier family } 1 \text { (high } \\
\text { affinity aspartate/glutamate } \\
\text { transporter), member } 6\end{array}$ & $-0,84$ & $1,88 \mathrm{E}-04$ \\
\hline Echdc2 & $\begin{array}{l}\text { enoyl Coenzyme A hydratase } \\
\text { domain containing } 2\end{array}$ & $-0,83$ & $9,43 \mathrm{E}-05$ \\
\hline Ccdc138 & $\begin{array}{l}\text { coiled-coil domain containing } \\
138\end{array}$ & $-0,83$ & $7,65 \mathrm{E}-06$ \\
\hline Galnt12 & $\begin{array}{c}\text { UDP-N-acetyl-alpha-D- } \\
\text { galactosamine:polypeptide } \mathrm{N} \text { - } \\
\text { acetylgalactosaminyltransferase } \\
12\end{array}$ & $-0,83$ & $8,22 \mathrm{E}-06$ \\
\hline Mina & myc induced nuclear antigen & $-0,83$ & $5,11 \mathrm{E}-05$ \\
\hline Slc35f5 & $\begin{array}{c}\text { solute carrier family } 35 \\
\text { member F5 }\end{array}$ & $-0,83$ & $1,22 \mathrm{E}-06$ \\
\hline Reep6 & receptor accessory protein 6 & $-0,82$ & $3,96 \mathrm{E}-06$ \\
\hline Gkap1 & G kinase anchoring protein 1 & $-0,82$ & $4,27 \mathrm{E}-05$ \\
\hline Pparg & $\begin{array}{c}\text { peroxisome proliferator } \\
\text { activated receptor gamma }\end{array}$ & $-0,82$ & $6,38 \mathrm{E}-06$ \\
\hline Gkap1 & G kinase anchoring protein 1 & $-0,81$ & $1,72 \mathrm{E}-05$ \\
\hline Gprc5c & $\begin{array}{l}\text { G protein-coupled receptor, } \\
\text { family } \mathrm{C} \text {, group } 5 \text {, member } \mathrm{C}\end{array}$ & $-0,78$ & $1,79 \mathrm{E}-04$ \\
\hline
\end{tabular}




\begin{tabular}{|c|c|c|c|}
\hline Asl & argininosuccinate lyase & $-0,78$ & $6,34 \mathrm{E}-05$ \\
\hline Churc1 & churchill domain containing 1 & $-0,77$ & $2,91 \mathrm{E}-04$ \\
\hline Gprc5c & $\begin{array}{l}\text { G protein-coupled receptor, } \\
\text { family } \mathrm{C} \text {, group } 5 \text {, member } \mathrm{C}\end{array}$ & $-0,77$ & $3,15 \mathrm{E}-04$ \\
\hline Peci & $\begin{array}{l}\text { peroxisomal delta3, delta2- } \\
\text { enoyl-Coenzyme A isomerase }\end{array}$ & $-0,76$ & $7,54 \mathrm{E}-06$ \\
\hline Prorsd1 & $\begin{array}{l}\text { prolyl-tRNA synthetase } \\
\text { domain containing } 1\end{array}$ & $-0,76$ & $1,74 \mathrm{E}-04$ \\
\hline Gm2954 & predicted gene 2954 & $-0,75$ & $1,40 \mathrm{E}-04$ \\
\hline BC016495 & cDNA sequence $\mathrm{BC} 016495$ & $-0,75$ & $8,10 \mathrm{E}-05$ \\
\hline Eda & ectodysplasin-A & $-0,75$ & $1,50 \mathrm{E}-04$ \\
\hline Kctd15 & $\begin{array}{l}\text { potassium channel } \\
\text { tetramerisation domain } \\
\text { containing } 15\end{array}$ & $-0,75$ & $3,52 \mathrm{E}-04$ \\
\hline Crlf2 & cytokine receptor-like factor 2 & $-0,74$ & $1,70 \mathrm{E}-04$ \\
\hline Ano10 & anoctamin 10 & $-0,74$ & $1,72 \mathrm{E}-04$ \\
\hline Sfxn4 & sideroflexin 4 & $-0,74$ & $1,49 \mathrm{E}-05$ \\
\hline Cdyl2 & $\begin{array}{l}\text { chromodomain protein, } \mathrm{Y} \\
\text { chromosome-like } 2\end{array}$ & $-0,72$ & $3,17 \mathrm{E}-05$ \\
\hline Smo & $\begin{array}{c}\text { smoothened homolog } \\
\text { (Drosophila) }\end{array}$ & $-0,71$ & $4,38 \mathrm{E}-06$ \\
\hline Tmem53 & transmembrane protein 53 & $-0,71$ & $1,66 \mathrm{E}-04$ \\
\hline Slc38a6 & $\begin{array}{c}\text { solute carrier family } 38, \\
\text { member } 6\end{array}$ & $-0,71$ & $2,77 \mathrm{E}-04$ \\
\hline Zfp105 & zinc finger protein 105 & $-0,71$ & $1,40 \mathrm{E}-04$ \\
\hline Ebf4 & early B-cell factor 4 & $-0,70$ & $1,53 \mathrm{E}-04$ \\
\hline Cdca7 & cell division cycle associated 7 & $-0,70$ & $1,66 \mathrm{E}-05$ \\
\hline Acadm & $\begin{array}{c}\text { acyl-Coenzyme A } \\
\text { dehydrogenase, medium chain }\end{array}$ & $-0,69$ & $1,31 \mathrm{E}-05$ \\
\hline Tpm4 & tropomyosin 4 & $-0,69$ & $2,17 \mathrm{E}-04$ \\
\hline Sh3bgrl2 & $\begin{array}{l}\text { SH3 domain binding glutamic } \\
\text { acid-rich protein like } 2\end{array}$ & $-0,67$ & $2,91 \mathrm{E}-04$ \\
\hline Cntln & centlein, centrosomal protein & $-0,67$ & $2,99 \mathrm{E}-05$ \\
\hline Cnga4 & $\begin{array}{c}\text { cyclic nucleotide gated channel } \\
\text { alpha } 4\end{array}$ & $-0,66$ & $2,98 \mathrm{E}-04$ \\
\hline Hddc2 & HD domain containing 2 & $-0,66$ & $5,03 \mathrm{E}-06$ \\
\hline Bend3 & BEN domain containing 3 & $-0,65$ & $9,88 \mathrm{E}-06$ \\
\hline Gpr125 & G protein-coupled receptor 125 & $-0,65$ & $2,30 \mathrm{E}-05$ \\
\hline Galk1 & galactokinase 1 & $-0,64$ & $2,41 E-04$ \\
\hline Pttg1 & $\begin{array}{l}\text { pituitary tumor-transforming } \\
\text { gene } 1\end{array}$ & $-0,64$ & 4,79E-05 \\
\hline Accs & $\begin{array}{l}\text { 1-aminocyclopropane-1- } \\
\text { carboxylate synthase homolog } \\
\text { (Arabidopsis)(non-functional) }\end{array}$ & $-0,64$ & $1,70 \mathrm{E}-04$ \\
\hline Ebpl & emopamil binding protein-like & $-0,63$ & $1,75 \mathrm{E}-05$ \\
\hline Trmt $2 b$ & $\begin{array}{l}\text { TRM2 tRNA methyltransferase } \\
2 \text { homolog B (S. cerevisiae) }\end{array}$ & $-0,63$ & $1,23 \mathrm{E}-05$ \\
\hline Zfp948 & zinc finger protein 948 & $-0,63$ & $2,86 \mathrm{E}-04$ \\
\hline Zfp945 & zinc finger protein 945 & $-0,63$ & $4,59 \mathrm{E}-05$ \\
\hline Ebi3 & Epstein-Barr virus induced & $-0,62$ & $2,14 \mathrm{E}-04$ \\
\hline
\end{tabular}




\begin{tabular}{|c|c|c|c|}
\hline & gene 3 & & \\
\hline Tmsb15b1 & thymosin beta $15 \mathrm{~b} 1$ & $-0,62$ & $8,01 \mathrm{E}-05$ \\
\hline Rangrf & $\begin{array}{l}\text { RAN guanine nucleotide } \\
\text { release factor }\end{array}$ & $-0,61$ & $8,53 \mathrm{E}-05$ \\
\hline Gusb & glucuronidase, beta & $-0,61$ & $2,18 \mathrm{E}-04$ \\
\hline Trpv2 & $\begin{array}{c}\text { transient receptor potential } \\
\text { cation channel, subfamily } \mathrm{V} \text {, } \\
\text { member } 2\end{array}$ & $-0,61$ & $4,01 \mathrm{E}-05$ \\
\hline Gm4535 & predicted gene 4535 & $-0,60$ & $2,12 \mathrm{E}-04$ \\
\hline Churc1 & churchill domain containing 1 & $-0,60$ & $1,05 \mathrm{E}-04$ \\
\hline Galnt12 & $\begin{array}{c}\text { UDP-N-acetyl-alpha-D- } \\
\text { galactosamine:polypeptide N- } \\
\text { acetylgalactosaminyltransferase } \\
12 \\
\end{array}$ & $-0,59$ & 2,78E-04 \\
\hline E2f1 & E2F transcription factor 1 & $-0,59$ & 9,37E-05 \\
\hline Cabyr & $\begin{array}{c}\text { calcium-binding tyrosine-(Y)- } \\
\text { phosphorylation regulated } \\
\text { (fibrousheathin 2) }\end{array}$ & 0,64 & $2,26 \mathrm{E}-04$ \\
\hline Ccdc88b & $\begin{array}{l}\text { coiled-coil domain containing } \\
88 \mathrm{~B}\end{array}$ & 0,66 & $1,01 \mathrm{E}-04$ \\
\hline Angpt1 & angiopoietin 1 & 0,70 & $3,75 \mathrm{E}-04$ \\
\hline Pcdhb22 & protocadherin beta 22 & 0,80 & $3,74 \mathrm{E}-07$ \\
\hline Ccdc88b & $\begin{array}{l}\text { coiled-coil domain containing } \\
\text { 88B }\end{array}$ & 0,95 & $1,46 \mathrm{E}-05$ \\
\hline Pcdhb21 & protocadherin beta 21 & 1,01 & $9,00 \mathrm{E}-06$ \\
\hline Krt9 & keratin 9 & 1,01 & $6,28 \mathrm{E}-06$ \\
\hline Chrdl2 & chordin-like 2 & 1,06 & $1,79 \mathrm{E}-05$ \\
\hline Megf6 & multiple EGF-like-domains 6 & 1,22 & 8,98E-05 \\
\hline Dach2 & dachshund 2 (Drosophila) & 3,43 & 2,90E-08 \\
\hline
\end{tabular}

Table 2: List of genes differentially regulated in CA region of F/F CKII mice

\begin{tabular}{|c|c|c|c|}
\hline Symbol & Name & $\begin{array}{c}\text { log (fold } \\
\text { change) }\end{array}$ & $\begin{array}{c}\text { Adjusted } \boldsymbol{p} \text { - } \\
\text { value }\end{array}$ \\
\hline Lor & loricrin & $-1,52$ & $1,51 \mathrm{E}-08$ \\
\hline Ccdc91 & $\begin{array}{c}\text { coiled-coil domain } \\
\text { containing 91 }\end{array}$ & $-1,02$ & $1,75 \mathrm{E}-07$ \\
\hline Dazl & $\begin{array}{c}\text { deleted in } \\
\text { azoospermia-like }\end{array}$ & $-2,71$ & $8,00 \mathrm{E}-07$ \\
\hline Prss16 & $\begin{array}{c}\text { protease, serine, } 16 \\
\text { (thymus) }\end{array}$ & $-3,41$ & $9,51 \mathrm{E}-07$ \\
\hline Zfp647 & $\begin{array}{c}\text { zinc finger protein } \\
\text { 647 }\end{array}$ & $-1,12$ & $1,59 \mathrm{E}-06$ \\
\hline Oxr1 & $\begin{array}{c}\text { oxidation } \\
\text { resistance 1 }\end{array}$ & $-0,92$ & $3,42 \mathrm{E}-06$ \\
\hline Parp11 & $\begin{array}{c}\text { poly (ADP-ribose) } \\
\text { polymerase family, } \\
\text { member 11 }\end{array}$ & $-0,83$ & $3,57 \mathrm{E}-06$ \\
\hline Reep6 & $\begin{array}{c}\text { receptor accessory } \\
\text { protein 6 }\end{array}$ & $-0,82$ & $4,26 \mathrm{E}-06$ \\
\hline
\end{tabular}




\begin{tabular}{|c|c|c|c|}
\hline Hdx & $\begin{array}{l}\text { highly divergent } \\
\text { homeobox }\end{array}$ & $-1,50$ & $6,37 \mathrm{E}-06$ \\
\hline Immp21 & $\begin{array}{c}\text { IMP2 inner } \\
\text { mitochondrial } \\
\text { membrane } \\
\text { peptidase-like (S. } \\
\text { cerevisiae) }\end{array}$ & $-0,92$ & $6,60 \mathrm{E}-06$ \\
\hline Hfm1 & $\begin{array}{c}\text { HFM1, ATP- } \\
\text { dependent DNA } \\
\text { helicase homolog } \\
\text { (S. cerevisiae) }\end{array}$ & $-1,78$ & $1,14 \mathrm{E}-05$ \\
\hline Wdr17 & $\begin{array}{c}\text { WD repeat domain } \\
17\end{array}$ & $-1,11$ & $1,12 \mathrm{E}-05$ \\
\hline Rimbp3 & $\begin{array}{l}\text { RIMS binding } \\
\text { protein } 3\end{array}$ & $-1,22$ & $2,76 \mathrm{E}-05$ \\
\hline Zbtb8a & $\begin{array}{l}\text { zinc finger and } \\
\text { BTB domain } \\
\text { containing } 8 \mathrm{a}\end{array}$ & $-1,29$ & $3,06 \mathrm{E}-05$ \\
\hline Ap1s3 & $\begin{array}{l}\text { adaptor-related } \\
\text { protein complex } \\
\text { AP-1, sigma } 3\end{array}$ & $-1,26$ & $3,69 \mathrm{E}-05$ \\
\hline Rabepk & $\begin{array}{c}\text { Rab9 effector } \\
\text { protein with kelch } \\
\text { motifs }\end{array}$ & $-0,87$ & $3,64 \mathrm{E}-05$ \\
\hline Cmya5 & $\begin{array}{l}\text { cardiomyopathy } \\
\text { associated } 5\end{array}$ & $-0,89$ & $4,09 \mathrm{E}-05$ \\
\hline Epb4.9 & $\begin{array}{l}\text { erythrocyte protein } \\
\text { band } 4.9\end{array}$ & $-0,44$ & $2,79 \mathrm{E}-05$ \\
\hline 4930403L05Rik & $\begin{array}{c}\text { protein } \\
\text { phosphatase } 1 \\
\text { regulatory subunit } \\
2 \text { pseudogene } \\
\end{array}$ & 1,92 & $2,07 \mathrm{E}-05$ \\
\hline Dach2 & $\begin{array}{l}\text { dachshund } 2 \\
\text { (Drosophila) }\end{array}$ & 3,04 & $1,26 \mathrm{E}-07$ \\
\hline
\end{tabular}




\section{Appendix 2:}

Table 3: List of differentially regulated genes in hippocampus of wild-type mice after 10 weeks of enrichment (the genes in red were confirmed by qRT-PCR)

\begin{tabular}{|c|c|c|c|}
\hline Symbol & Name & $\begin{array}{l}\text { log (fold } \\
\text { change) }\end{array}$ & $\begin{array}{c}\text { Adjusted } \\
p \text {-value }\end{array}$ \\
\hline Lrriq4 & $\begin{array}{c}\text { leucine-rich repeats and IQ } \\
\text { motif containing } 4\end{array}$ & 3,71 & $1,46 \mathrm{E}-04$ \\
\hline Cuedc1 & CUE domain containing 1 & 3,31 & $2,75 \mathrm{E}-08$ \\
\hline Slfn13 & schlafen family member 13 & 3,12 & $2,74 \mathrm{E}-10$ \\
\hline Pdxdc1 & $\begin{array}{l}\text { pyridoxal-dependent } \\
\text { decarboxylase domain } \\
\text { containing } 1\end{array}$ & 2,74 & $3,03 \mathrm{E}-07$ \\
\hline Gm3219 & $\begin{array}{l}\text { B-cell CLL/lymphoma 7C } \\
\text { pseudogene }\end{array}$ & 2,68 & $6,39 \mathrm{E}-06$ \\
\hline Pde5a & $\begin{array}{l}\text { phosphodiesterase 5A, cGMP- } \\
\text { specific }\end{array}$ & 2,36 & $1,98 \mathrm{E}-04$ \\
\hline Pcdhb2 & protocadherin beta 2 & 2,07 & $1,74 \mathrm{E}-06$ \\
\hline S1fn10-ps & schlafen 10 , pseudogene & 2,02 & $1,16 \mathrm{E}-06$ \\
\hline Plbd1 & $\begin{array}{l}\text { phospholipase B domain } \\
\text { containing } 1\end{array}$ & 1,63 & $1,99 \mathrm{E}-04$ \\
\hline Tjp2 & tight junction protein 2 & 1,62 & $6,03 \mathrm{E}-08$ \\
\hline Tmco6 & $\begin{array}{c}\text { transmembrane and coiled-coil } \\
\text { domains } 6\end{array}$ & 1,59 & $4,17 \mathrm{E}-06$ \\
\hline Ccl6 & $\begin{array}{c}\text { chemokine (C-C motif) ligand } \\
6 \\
\end{array}$ & 1,40 & $5,34 \mathrm{E}-07$ \\
\hline Paip1 & $\begin{array}{l}\text { polyadenylate binding protein- } \\
\text { interacting protein } 1\end{array}$ & 1,39 & $1,10 \mathrm{E}-05$ \\
\hline Nnt & $\begin{array}{l}\text { nicotinamide nucleotide } \\
\text { transhydrogenase }\end{array}$ & 1,24 & $2,30 \mathrm{E}-04$ \\
\hline Kank1 & $\begin{array}{l}\mathrm{KN} \text { motif and ankyrin repeat } \\
\text { domains } 1\end{array}$ & 1,15 & 4,84E-08 \\
\hline $\mathrm{Cml5}$ & camello-like 5 & 1,14 & $7,19 \mathrm{E}-05$ \\
\hline Mfi2 & $\begin{array}{c}\text { antigen p97 (melanoma } \\
\text { associated) identified by } \\
\text { monoclonal antibodies } 133.2 \\
\text { and } 96.5\end{array}$ & 1,13 & 4,59E-07 \\
\hline Dap3 & death associated protein 3 & 1,09 & $3,92 \mathrm{E}-04$ \\
\hline Slc26a10 & $\begin{array}{c}\text { solute carrier family } 26, \\
\text { member } 10\end{array}$ & 1,05 & $4,02 \mathrm{E}-04$ \\
\hline $\operatorname{Pdzd} 2$ & PDZ domain containing 2 & 1,05 & 2,77E-04 \\
\hline $\mathrm{Ccl} 8$ & $\begin{array}{c}\text { chemokine (C-C motif) ligand } \\
8\end{array}$ & 1,05 & $4,51 \mathrm{E}-04$ \\
\hline Med1 & mediator complex subunit 1 & 1,03 & $2,67 \mathrm{E}-06$ \\
\hline Pdxdc1 & $\begin{array}{c}\text { pyridoxal-dependent } \\
\text { decarboxylase domain } \\
\text { containing } 1\end{array}$ & 1,01 & $8,62 \mathrm{E}-05$ \\
\hline $\operatorname{Sec} 24 d$ & $\begin{array}{l}\text { Sec24 related gene family, } \\
\text { member D (S. cerevisiae) }\end{array}$ & 1,00 & $1,83 \mathrm{E}-04$ \\
\hline Ptar1 & protein prenyltransferase alpha & 0,98 & $4,19 \mathrm{E}-05$ \\
\hline
\end{tabular}




\begin{tabular}{|c|c|c|c|}
\hline & subunit repeat containing 1 & & \\
\hline Slc15a2 & $\begin{array}{c}\text { solute carrier family } 15 \\
(\mathrm{H}+/ \text { peptide transporter }) \\
\text { member } 2\end{array}$ & 0,97 & $1,23 \mathrm{E}-06$ \\
\hline Cdk15 & cyclin-dependent kinase-like 5 & 0,95 & $1,04 \mathrm{E}-04$ \\
\hline Pgm5 & phosphoglucomutase 5 & 0,94 & $3,24 \mathrm{E}-06$ \\
\hline Syce2 & $\begin{array}{l}\text { synaptonemal complex central } \\
\text { element protein } 2\end{array}$ & 0,84 & $1,38 \mathrm{E}-04$ \\
\hline Impg2 & $\begin{array}{l}\text { interphotoreceptor matrix } \\
\text { proteoglycan } 2\end{array}$ & 0,81 & $3,08 \mathrm{E}-04$ \\
\hline Acvr1c & activin A receptor, type IC & 0,80 & $1,87 \mathrm{E}-04$ \\
\hline Dcpp3 & $\begin{array}{l}\text { demilune cell and parotid } \\
\text { protein } 3 \\
\end{array}$ & 0,80 & $2,30 \mathrm{E}-04$ \\
\hline Nkd1 & $\begin{array}{l}\text { naked cuticle } 1 \text { homolog } \\
\text { (Drosophila) }\end{array}$ & 0,78 & $3,14 \mathrm{E}-04$ \\
\hline Nsa2 & $\begin{array}{l}\text { NSA2 ribosome biogenesis } \\
\text { homolog (S. cerevisiae) }\end{array}$ & 0,78 & $1,63 \mathrm{E}-06$ \\
\hline Rnls & $\begin{array}{l}\text { renalase, FAD-dependent } \\
\text { amine oxidase }\end{array}$ & 0,78 & $3,08 \mathrm{E}-04$ \\
\hline Cbwd1 & COBW domain containing 1 & 0,77 & $3,75 \mathrm{E}-06$ \\
\hline Camk4 & $\begin{array}{l}\text { calcium/calmodulin-dependent } \\
\text { protein kinase IV }\end{array}$ & 0,76 & $6,80 \mathrm{E}-05$ \\
\hline Mfsd6 & $\begin{array}{c}\text { major facilitator superfamily } \\
\text { domain containing } 6\end{array}$ & 0,75 & $5,31 \mathrm{E}-04$ \\
\hline Jakmip3 & $\begin{array}{l}\text { janus kinase and microtubule } \\
\text { interacting protein } 3\end{array}$ & 0,73 & $3,99 \mathrm{E}-04$ \\
\hline Fastk & $\begin{array}{c}\text { Fas-activated serine/threonine } \\
\text { kinase }\end{array}$ & 0,73 & $3,28 \mathrm{E}-04$ \\
\hline Galnt11 & $\begin{array}{c}\text { UDP-N-acetyl-alpha-D- } \\
\text { galactosamine:polypeptide N- } \\
\text { acetylgalactosaminyltransferase } \\
11 \\
\end{array}$ & 0,72 & $8,70 \mathrm{E}-05$ \\
\hline Ing3 & $\begin{array}{l}\text { inhibitor of growth family, } \\
\text { member } 3\end{array}$ & 0,72 & $4,94 \mathrm{E}-05$ \\
\hline Tmem2 & transmembrane protein 2 & 0,72 & $1,81 \mathrm{E}-04$ \\
\hline Otos & otospiralin & 0,70 & $3,62 \mathrm{E}-05$ \\
\hline Stard4 & $\begin{array}{l}\text { StAR-related lipid transfer } \\
\text { (START) domain containing } 4\end{array}$ & 0,70 & $2,33 \mathrm{E}-05$ \\
\hline Robo1 & $\begin{array}{c}\text { roundabout homolog } 1 \\
\text { (Drosophila) }\end{array}$ & 0,69 & $7,24 \mathrm{E}-05$ \\
\hline Ptgs2 & $\begin{array}{c}\text { prostaglandin-endoperoxide } \\
\text { synthase } 2\end{array}$ & 0,67 & $4,11 \mathrm{E}-05$ \\
\hline Gm16500 & predicted gene 16500 & 0,67 & $1,02 \mathrm{E}-04$ \\
\hline A930018M24Rik & $\begin{array}{l}\text { RIKEN cDNA A930018M24 } \\
\text { gene }\end{array}$ & 0,67 & $3,58 \mathrm{E}-04$ \\
\hline LOC100504473 & $\begin{array}{l}\text { RNA-binding protein Musashi } \\
\text { homolog 2-like }\end{array}$ & 0,66 & $1,64 \mathrm{E}-04$ \\
\hline Smek2 & $\begin{array}{l}\text { SMEK homolog 2, suppressor } \\
\text { of mek1 (Dictyostelium) }\end{array}$ & 0,66 & 2,89E-04 \\
\hline Gabra2 & $\begin{array}{l}\text { gamma-aminobutyric acid } \\
\text { (GABA) A receptor, subunit } \\
\text { alpha } 2\end{array}$ & 0,64 & $1,01 \mathrm{E}-04$ \\
\hline Xbp1 & X-box binding protein 1 & 0,62 & $3,88 \mathrm{E}-04$ \\
\hline
\end{tabular}




\begin{tabular}{|c|c|c|c|}
\hline Eif2c4 & $\begin{array}{l}\text { eukaryotic translation initiation } \\
\text { factor } 2 \mathrm{C}, 4\end{array}$ & 0,62 & $1,96 \mathrm{E}-04$ \\
\hline A730020M07Rik & $\begin{array}{c}\text { RIKEN cDNA A730020M07 } \\
\text { gene }\end{array}$ & 0,61 & $3,74 \mathrm{E}-04$ \\
\hline Mrgpra2b & $\begin{array}{l}\text { MAS-related GPR, member } \\
\text { A2B }\end{array}$ & 0,61 & $1,98 \mathrm{E}-04$ \\
\hline Fam150b & $\begin{array}{l}\text { family with sequence similarity } \\
150, \text { member B }\end{array}$ & 0,61 & $2,40 \mathrm{E}-04$ \\
\hline Meox1 & mesenchyme homeobox 1 & 0,60 & $4,08 \mathrm{E}-04$ \\
\hline Crebl2 & $\begin{array}{l}\text { cAMP responsive element } \\
\text { binding protein-like } 2\end{array}$ & 0,59 & $2,60 \mathrm{E}-04$ \\
\hline Hist1h2bc & histone cluster 1, H2bc & 0,59 & $8,72 \mathrm{E}-05$ \\
\hline Tctex $1 \mathrm{~d} 2$ & Tctex 1 domain containing 2 & 0,59 & $8,76 \mathrm{E}-06$ \\
\hline Kat2a & $\mathrm{K}$ (lysine) acetyltransferase 2A & $-0,58$ & $5,78 \mathrm{E}-04$ \\
\hline Pisd-ps3 & $\begin{array}{c}\text { phosphatidylserine } \\
\text { decarboxylase, pseudogene } 3\end{array}$ & $-0,58$ & $1,11 \mathrm{E}-04$ \\
\hline Rab5b & $\begin{array}{c}\text { RAB5B, member RAS } \\
\text { oncogene family }\end{array}$ & $-0,58$ & $3,46 \mathrm{E}-05$ \\
\hline Prr12 & proline rich 12 & $-0,59$ & $1,06 \mathrm{E}-04$ \\
\hline 38961 & septin 6 & $-0,59$ & $6,60 \mathrm{E}-04$ \\
\hline Gm4072 & predicted gene 4072 & $-0,60$ & $5,36 \mathrm{E}-04$ \\
\hline Cnksr1 & $\begin{array}{c}\text { connector enhancer of kinase } \\
\text { suppressor of Ras } 1\end{array}$ & $-0,60$ & $3,82 \mathrm{E}-04$ \\
\hline Pik3r2 & $\begin{array}{l}\text { phosphatidylinositol 3-kinase, } \\
\text { regulatory subunit, polypeptide } \\
2 \text { (p85 beta) }\end{array}$ & $-0,61$ & $2,16 \mathrm{E}-05$ \\
\hline Sncb & synuclein, beta & $-0,61$ & $6,02 \mathrm{E}-04$ \\
\hline Gm7109 & predicted gene 7109 & $-0,63$ & $9,87 \mathrm{E}-05$ \\
\hline Prepl & prolyl endopeptidase-like & $-0,63$ & $2,10 \mathrm{E}-04$ \\
\hline Grin2b & $\begin{array}{c}\text { glutamate receptor, ionotropic, } \\
\text { NMDA2B (epsilon 2) }\end{array}$ & $-0,63$ & $2,30 \mathrm{E}-04$ \\
\hline Smarcd1 & $\begin{array}{l}\text { SWI/SNF related, matrix } \\
\text { associated, actin dependent } \\
\text { regulator of chromatin, } \\
\text { subfamily d, member } 1 \\
\end{array}$ & $-0,63$ & $1,65 \mathrm{E}-04$ \\
\hline Mlycd & malonyl-CoA decarboxylase & $-0,65$ & $4,48 \mathrm{E}-04$ \\
\hline Kif1c & kinesin family member $1 \mathrm{C}$ & $-0,65$ & $1,64 \mathrm{E}-04$ \\
\hline Glod4 & glyoxalase domain containing 4 & $-0,67$ & 2,94E-04 \\
\hline Chchd10 & $\begin{array}{l}\text { coiled-coil-helix-coiled-coil- } \\
\text { helix domain containing } 10\end{array}$ & $-0,67$ & $7,06 \mathrm{E}-05$ \\
\hline Ssx2ip & $\begin{array}{l}\text { synovial sarcoma, } \mathrm{X} \text { breakpoint } \\
2 \text { interacting protein } \\
\end{array}$ & $-0,68$ & $3,33 \mathrm{E}-04$ \\
\hline Rps6kb1 & $\begin{array}{c}\text { ribosomal protein S6 kinase, } \\
\text { polypeptide } 1\end{array}$ & $-0,68$ & $1,81 \mathrm{E}-06$ \\
\hline Erbb3 & $\begin{array}{c}\text { v-erb-b2 erythroblastic } \\
\text { leukemia viral oncogene } \\
\text { homolog } 3 \text { (avian) }\end{array}$ & $-0,69$ & $5,70 \mathrm{E}-04$ \\
\hline Gm3652 & predicted gene 3652 & $-0,69$ & $4,37 \mathrm{E}-04$ \\
\hline Dgke & diacylglycerol kinase, epsilon & $-0,69$ & $1,74 \mathrm{E}-05$ \\
\hline Pcdhb4 & protocadherin beta 4 & $-0,70$ & 5,94E-05 \\
\hline Asnal & arsA arsenite transporter, ATP- & $-0,71$ & $1,83 \mathrm{E}-04$ \\
\hline
\end{tabular}




\begin{tabular}{|c|c|c|c|}
\hline & binding, homolog 1 (bacterial) & & \\
\hline Lipc & lipase, hepatic & $-0,71$ & $2,73 \mathrm{E}-04$ \\
\hline Gm5151 & predicted gene 5151 & $-0,73$ & $3,94 \mathrm{E}-04$ \\
\hline Olfm1 & olfactomedin 1 & $-0,73$ & $1,06 \mathrm{E}-04$ \\
\hline Ssbp4 & $\begin{array}{l}\text { single stranded DNA binding } \\
\text { protein } 4\end{array}$ & $-0,73$ & $1,31 \mathrm{E}-04$ \\
\hline Nsg2 & $\begin{array}{l}\text { neuron specific gene family } \\
\text { member } 2\end{array}$ & $-0,75$ & $8,67 \mathrm{E}-05$ \\
\hline Golt1a & $\begin{array}{c}\text { golgi transport } 1 \text { homolog A } \\
\text { (S. cerevisiae) }\end{array}$ & $-0,75$ & $5,08 \mathrm{E}-04$ \\
\hline D8Ertd738e & $\begin{array}{c}\text { DNA segment, Chr 8, ERATO } \\
\text { Doi } 738, \text { expressed }\end{array}$ & $-0,77$ & $2,36 \mathrm{E}-04$ \\
\hline Mcm9 & $\begin{array}{l}\text { minichromosome maintenance } \\
\text { complex component } 9\end{array}$ & $-0,78$ & $3,08 \mathrm{E}-04$ \\
\hline $\operatorname{Cdc} 23$ & $\begin{array}{c}\text { CDC23 (cell division cycle 23, } \\
\text { yeast, homolog) }\end{array}$ & $-0,78$ & $1,08 \mathrm{E}-04$ \\
\hline Hdgf & $\begin{array}{c}\text { hepatoma-derived growth } \\
\text { factor }\end{array}$ & $-0,78$ & $1,09 \mathrm{E}-04$ \\
\hline Smarce1 & $\begin{array}{l}\text { SWI/SNF related, matrix } \\
\text { associated, actin dependent } \\
\text { regulator of chromatin, } \\
\text { subfamily e, member } 1\end{array}$ & $-0,78$ & $2,13 \mathrm{E}-05$ \\
\hline Zfp961 & zinc finger protein 961 & $-0,79$ & $9,50 \mathrm{E}-05$ \\
\hline Tom1 & $\begin{array}{c}\text { target of myb1 homolog } \\
\text { (chicken) }\end{array}$ & $-0,79$ & 4,97E-04 \\
\hline Accn3 & $\begin{array}{c}\text { amiloride-sensitive cation } \\
\text { channel } 3\end{array}$ & $-0,81$ & $2,66 \mathrm{E}-04$ \\
\hline Pcgf2 & polycomb group ring finger 2 & $-0,82$ & $6,46 \mathrm{E}-04$ \\
\hline Gfap & glial fibrillary acidic protein & $-0,92$ & $1,93 \mathrm{E}-04$ \\
\hline Pi4kb & $\begin{array}{l}\text { phosphatidylinositol 4-kinase, } \\
\text { catalytic, beta polypeptide }\end{array}$ & $-0,92$ & $7,58 \mathrm{E}-05$ \\
\hline Gadd45gip1 & $\begin{array}{l}\text { growth arrest and DNA- } \\
\text { damage-inducible, gamma } \\
\text { interacting protein } 1\end{array}$ & $-0,93$ & 2,69E-04 \\
\hline Lpl & lipoprotein lipase & $-0,94$ & $4,21 \mathrm{E}-04$ \\
\hline Olfr678 & olfactory receptor 678 & $-0,96$ & $5,57 \mathrm{E}-04$ \\
\hline Hapln4 & $\begin{array}{l}\text { hyaluronan and proteoglycan } \\
\text { link protein } 4\end{array}$ & $-0,98$ & $2,25 \mathrm{E}-04$ \\
\hline Plvap & $\begin{array}{c}\text { plasmalemma vesicle } \\
\text { associated protein }\end{array}$ & $-1,00$ & 4,69E-06 \\
\hline Apold1 & $\begin{array}{l}\text { apolipoprotein L domain } \\
\text { containing } 1\end{array}$ & $-1,03$ & $1,67 \mathrm{E}-05$ \\
\hline Hdgf & $\begin{array}{l}\text { hepatoma-derived growth } \\
\text { factor }\end{array}$ & $-1,05$ & $1,84 \mathrm{E}-05$ \\
\hline Fam32a & $\begin{array}{c}\text { family with sequence similarity } \\
32, \text { member A }\end{array}$ & $-1,05$ & $1,64 \mathrm{E}-04$ \\
\hline Plvap & $\begin{array}{c}\text { plasmalemma vesicle } \\
\text { associated protein }\end{array}$ & $-1,10$ & $8,01 \mathrm{E}-05$ \\
\hline Stac2 & $\begin{array}{l}\text { SH3 and cysteine rich domain } \\
2\end{array}$ & $-1,10$ & $1,18 \mathrm{E}-05$ \\
\hline Lpcat2 & $\begin{array}{c}\text { lysophosphatidylcholine } \\
\text { acyltransferase } 2\end{array}$ & $-1,15$ & $5,49 \mathrm{E}-04$ \\
\hline Vmn1r90 & vomeronasal 1 receptor 90 & $-1,15$ & $2,72 \mathrm{E}-04$ \\
\hline
\end{tabular}




\begin{tabular}{|c|c|c|c|}
\hline Lpl & lipoprotein lipase & $-1,17$ & $2,32 \mathrm{E}-05$ \\
\hline $\mathrm{Il12 \textrm {rb } 1}$ & interleukin 12 receptor, beta 1 & $-1,18$ & $2,77 \mathrm{E}-04$ \\
\hline Camk4 & $\begin{array}{l}\text { calcium/calmodulin-dependent } \\
\text { protein kinase IV }\end{array}$ & $-1,22$ & $2,07 \mathrm{E}-05$ \\
\hline Klf1 & $\begin{array}{c}\text { Kruppel-like factor } 1 \\
\text { (erythroid) }\end{array}$ & $-1,34$ & $9,11 \mathrm{E}-05$ \\
\hline Ccl17 & $\begin{array}{l}\text { chemokine (C-C motif) ligand } \\
17\end{array}$ & $-1,36$ & $1,07 \mathrm{E}-05$ \\
\hline Slfn2 & schlafen 2 & $-1,49$ & $6,23 \mathrm{E}-06$ \\
\hline Zfp963 & zinc finger protein 963 & $-1,52$ & $9,23 \mathrm{E}-06$ \\
\hline Def8 & $\begin{array}{c}\text { differentially expressed in } \\
\text { FDCP } 8\end{array}$ & $-1,52$ & $1,13 \mathrm{E}-04$ \\
\hline Mamdc2 & MAM domain containing 2 & $-1,96$ & $8,76 \mathrm{E}-07$ \\
\hline Capns2 & calpain, small subunit 2 & $-2,07$ & $1,43 \mathrm{E}-04$ \\
\hline Fastk & $\begin{array}{c}\text { Fas-activated serine/threonine } \\
\text { kinase }\end{array}$ & $-2,10$ & $8,34 \mathrm{E}-09$ \\
\hline Pde6h & $\begin{array}{c}\text { phosphodiesterase } 6 \mathrm{H}, \mathrm{cGMP}- \\
\text { specific, cone, gamma }\end{array}$ & $-2,29$ & $2,33 \mathrm{E}-05$ \\
\hline Ocel1 & $\begin{array}{c}\text { occludin/ELL domain } \\
\text { containing } 1\end{array}$ & $-2,92$ & $9,23 \mathrm{E}-05$ \\
\hline Tbx4 & T-box 4 & $-3,46$ & $1,44 \mathrm{E}-08$ \\
\hline Rheb & Ras homolog enriched in brain & $-4,79$ & $6,27 \mathrm{E}-10$ \\
\hline Krt12 & keratin 12 & $-6,60$ & $4,66 \mathrm{E}-08$ \\
\hline
\end{tabular}




\section{ACKNOWLEDGEMENTS}

I would like to express my gratitude to Prof. Dr. Andre Fischer for providing me with the opportunity to carry out these highly interesting projects and his close supervision. My thanks also go to Farah and Tanja (former PhD student in the lab) for their assistance during my first year in the lab. My special thanks go to Sanaz for her help in the lab and most importantly for her support and advice during my hard times (scientific and nonscientific alike), and being a very good friend in general. Also my thanks go to Roberto for helping me a lot with experiments - especially with isolating dorsal and ventral portions of dentate gyrus and CA, which proved to be crucial for my research. Also I would like to thank Torsten for proofreading parts of my thesis and his help in formatting it. My thanks go to Pooja, Jerzy, Rashi and Eva for the chats (better call them 'monologs') that I had during the breaks that I made while writing my thesis and for listening to me patiently. I would like to thank Roman for scientific discussions that we had. I wish Hope all the best in his future endeavours and hope that he will finish his medical studies with success and in the end he will be with his decision to leave science and pursue a medical career. I would also like to thank Sergej for being a good and patient 'office mate' and the chats and great laughs that we had in the lab during the breaks. I would like to thank Susanne for her help in some of my experiments as well. Moreover, I would like to wish all the best to our former lab members Kim, Perla, Govind and Athanassis.

Obviously I would also like to express my gratitude to my parents firstly for transmitting to me their genes that endowed me with the capability to carry out my studies and this highly interesting and complicated research in particular, and secondly for supporting me in my decision to utilize my endowment in the direction that I have chosen. My special thanks go to my brother, who is also currently doing his $\mathrm{PhD}$ in Germany, in chemical engineering in RWTH Aachen, for his close presence and for his being a great friend. We frequently met and travelled a lot together around Europe and had a great time. Moreover, I would like to thank Andrius, Olga and Lena for being great friends and flatmates during my stay in Goettingen, for creating a cheerful, warm and family-like 
atmosphere and for all the activities (travelling, playing etc.) that we did together. Living with them in the same dorm was a great pleasure and knowing people like them was a special experience in the first place. What was especially valuable was the feeling of return to a warm and friendly place after a hard working day.

Obviously, I am grateful to IMPRS Program in Neuroscience for providing me with the opportunity of carrying out my $\mathrm{PhD}$ research in Germany, for Prof. Dr. Michael Hoerner and Sandra Drube for their organizational skills and supervision and again IMPRS, GGNB and Medical Faculty of Goettingen University for supporting me financially during my $\mathrm{PhD}$. 


\section{Curriculum Vitae}

\section{PERSONAL DETAILS}

Name: Cemil Kerimoglu

Birthplace: Baku, Azerbaijan

Date of Birth: $26^{\text {th }}$ August 1984

\section{EDUCATION}

Oct. 2008 - current

$\mathrm{PhD}$ study in the Laboratory for Aging and Cognitive Diseases, Dr. Andre Fischer in European Neuroscience Institute (ENI), Göttingen / GERMANY

$2007-2008$ MSc student in IMPRS MSc/PhD/MD-PhD Program in Neurosciences, Göttingen / GERMANY

$2003-2007$

BSc in Middle East Technical University (METU) Department of Molecular Biology and Genetics, Ankara / TURKEY

\section{SCHOLARSHIPS}

2007 - 2008: Stipend of the Excellence Foundation for the Promotion of the Max Planck Society

Summer 2006: British Genetics Society Summer Studentship, UK

\section{RESEARCH}

Oct. 2008 - current Doctoral project: Role of Histone Methylation in Cognitive Function and Molecular Mechanisms Mediating the Effect of Environmental Enrichment on Learning \& Memory 


\section{PUBLICATIONS}

Bolender J., Erdeniz B., Kerimoglu C.; "Hominid Uniqueness, Cognition by Description and Procedural Memory"; Biolinguistics, 2008

Baharijavan S., Maddalena A.*, Kerimoglu C.*, Wittnam J., Held T., Bähr M., Burkhardt S., Delalle I., Kügler S., Fischer A. and Sananbenesi F.; "HDAC1 Regulates Fear Extinction in Mice" (accepted for publication from Journal of Neuroscience)

* equal contribtion

Agis-Balboa R.C., Pavelka Z., Kerimoglu C., Sanabenesi F., Fischer A.; “Targeting Hdac5 in the Alzheimer's disease animal model APPPS1-21 is detrimental for cognition" (submitted to Journal of Alzheimer's Disease)

Kerimoglu C., Agis-Balboa R.C., Kranz A., Burkhardt S., Baharijavan S., Opitz L., SalinasRiester G., Fischer A. "MLL2-mediated histone methylation in dentate gyrus is important for learning \& memory in mice" (in preparation)

Kerimoglu C., Stilling R., Rao P., Agis-Balboa R.C., Fischer A. "Persistence of the effects of environmental enrichment depends on its duration" (in preparation)

Kerimoglu C., Baharijavan S., Fischer A. "Environmental enrichment dampens the response to a novel stimulus" (in preparation) 